\title{
Analyzing the Impact of COVID-19 on the Financial Failure Risk in Borsa İstanbul Manufacturing Companies
}

\author{
Emre KAPLANOĞLU ${ }^{\mathrm{a}, *}$, Fatma MOROĞLU ${ }^{\mathrm{b}}$ \\ a Assoc. Prof., Ege University, Bergama Technical and Business College, Accounting and Tax Application Program, Turkey \\ ${ }^{\mathrm{b}}$ Lect., Ege University, Bergama Technical and Business College, Accounting and Tax Application Program, Turkey
}

\section{A B S T R A C T}

There is no agreed and precise definition of the concept of financial failure. This situation causes the studies of the concept to be associated with bankruptcies. Although not every company experiencing financial failure goes bankrupt, it can be noted that economic fluctuations that happen on a global scale cause many companies to face the risk of financial failure and even bankruptcy. Furthermore, the COVID-19 pandemic has also affected the economic policies of countries and thus affected the operations of companies. This study aims to analyze the financial failure risk of Borsa İstanbul (BIST) manufacturing companies before and after COVID-19. In the research, financial statements of BIST manufacturing industry companies published quarterly between the years 2019-2020 were used. Within the scope of the research, the quarterly financial statements of 146 BIST companies listed in the manufacturing industry for the years 2019-2020 were analyzed with the financial failure models of Altman (1968), Springate (1978), Taffler (1983) and Zmijewski (1984).
\end{abstract}

\author{
ARTICLE INFO \\ Keywords: \\ Borsa İstanbul, COVID-19, Financial \\ Failure, Manufacturing, Risk
}

*Corresponding author:

emre.kaplanoglu@ege.edu.tr

Emre KAPLANOĞLU

\section{Article history:}

Received : 09.10.2021

Revised : 15.11.2021

Accepted : 29.11.2021

DOI: https://doi.org/10.51410/jcgirm.8.2.7

\section{INTRODUCTION}

In recent years, the deepening of global trade and currency wars and the increase in protectionist concerns in international trade has adversely affected the economies of many developed and developing countries and businesses operating in these economies. In this context, states and businesses may experience fluctuations in the economic and financial context. While the results of these adverse effects can often be measured within specific methods, they are sometimes insufficient due to excessive uncertainty, which may increase risk. The global impact of the COVID-19 pandemic, which emerged in Wuhan, China, in December 2019 and spread worldwide in a short time, presents much uncertainty and, therefore, risk.

Pandemics have been occurring naturally since ancient times. A highly pathogenic and antigenically exceptional novel type A virus has spread easily to and from humans. Although the virus was not recognized until 1933, historical records describe pandemics dating back to Hippocrates. The first severe pandemic with vital historical records occurred in 1580 and was determined to have destroyed some Spanish cities. Ten pandemic outbreaks have been documented in the last 300 years (Kelley \& 
Osterholm, 2008). However, it is controversial that the recent COVID-19 pandemic will cause radical changes in the current order. These changes also constitute a source of risk.

From a financial point of view, it is of great importance for businesses to keep their costs and expenses related to production, activities and financing under control, to closely monitor current and potential interest, exchange rate and liquidity risks, and to make their cash flows and profitability sustainable in order to avoid financial failure. It can be said that almost all sectors have been adversely affected by the COVID-19 process. Prominent sectors can be listed as follows: Airlines, hotels, restaurants, hospitality services, retail, especially manufacturers with complex supply chains, exporters heavily dependent on the Asian market first affected by the global pandemic, tourism-related businesses, transportation, cruises, ports, and the shipping sector, due to the decline in demand and commodity prices for oil, gas, mining, and metal industries. The problems companies faced during the COVID-19 period can be listed as follows: Consumer demand has decreased, and it is uncertain when it will increase and return to its former level. Companies' supply chain has been disrupted, and cash and working capital problems have emerged. Suppliers have had difficulty delivering critical components to manufacturers, and the production process has been delayed or stopped. The decline in consumer demand has caused a backlog in the stocks of companies, and it has become more and more challenging to clean their inventories. As a result, companies had difficulties collecting their receivables from their cash-strapped customers on time. There were also delays in supplier payments due to short-term cash flow constraints. Posted checks, which play a critical role in commercial life and are used as a method of financing receivables, caused serious collection problems due to the cash flow problems in this period. In addition, since companies guarantee post-dated checks, financing problems and their legal consequences arise due to the inability to pay post-dated checks (Deloitte, 2020). Therefore, the risk of financial failure increases due to these problems faced by companies during the COVID-19 process.

This study aims to analyze the financial failure risk of companies listed in the Borsa İstanbul (BIST) manufacturing industry in 2019 and 2020 comparatively. The financial data of the companies for the years 2019 and 2020 was analyzed quarterly. Within the scope of the research, the quarterly financial statements of 146 BIST companies listed in the manufacturing industry sector for the years 2019-2020 were evaluated using Altman (1968), Springate (1978), Taffler (1983) and Zmijewski (1984) financial failure models. The study consists of five main parts. In the introduction part of the study, the relationship between the risk of financial failure and the COVID-19 pandemic and the purpose of the study is explained. In the second part, the literature review on financial failure is presented, and in the third part, the scope and method of the study are given. In the fourth part, the analysis findings made according to the financial failure models of BIST manufacturing industry companies are presented. Finally, in the fifth part, the analysis findings are explained by comparing them with the literature and sharing suggestions for future studies. 


\section{LITERATURE REVIEW}

Winakor \& Smith (1935), one of the first financial failure modelling studies, examined 183 companies that went bankrupt between 1923 and 1931. Merwin (1942), on the other hand, examined the financial ratios of firms before bankruptcy to their financial failures over the data of a total of 900 firms that went bankrupt and continued their activities in the period 1926-1936. In both studies, net working capital ratio (net working capital/total assets) and current ratio were important variables in estimating financial failure.

Beaver (1966) conducted one of the most widely known studies among univariate financial failure models, including 79 successful and 79 unsuccessful companies listed in the USA between 1954 and 1964. Weibel (1973) studied 36 successful and 79 unsuccessful firms listed in Switzerland during the 1960-1971 period. They identified the most successful variables in estimating financial failure by using the financial data of 36 unsuccessful small-scale firms. Beaver (1966) found that the working capital ratio (working capital/total assets) and current ratio are among the most influential variables in predicting financial failure, while Weibel (1973) found that the current ratio is among the most influential variables in predicting financial failure.

The first multivariate financial failure model experiments were carried out by Tamari (1966) using the financial data for the period 1956-1960 and included 16 firms that filed for bankruptcy and 12 Israeli firms that went bankrupt. As a result of the study, it has been determined that the current ratio of the companies is one of the six variables that affect their financial failures. Among the financial failure models, the most widely known studies are Altman's (1968; 1983; 1993) studies. Among these, Altman's (1968) study has the feature of being the first financial failure study that does not contain personal judgments and is based entirely on statistical methods. In his study, Altman (1968) developed an estimation model based on the main variables affecting financial failure by using the data of 33 successful and 33 unsuccessful publicly traded manufacturing firms listed in the USA with multiple discriminant analyses. At the end of the study, five financial ratios, including the net working capital ratio, were selected among 22 financial ratios for the model called Z score. Altman (1983) developed the $\mathrm{Z}$ score model, which is a model that can also be used in non-public companies, and Altman (1993) altered the Z score model, which can be used in both publicly traded companies and companies listed in industries other than manufacturing.

Springate (1978) studied 40 manufacturing companies listed in Canada; Ohlson (1980) used 105 unsuccessful and 2058 successful companies listed in the USA; and Taffler (1982) examined 25 unsuccessful and 45 successful companies trading on the London Stock Exchange. They aimed to determine the financial ratios that affect financial failure by creating a model that could be used to predict financial failure. All three models have determined that the working capital ratio affects financial failure, which is included in the estimation models. In another study to develop a financial failure model, Zmijewski (1984) developed 12 models using data between 1972 and 1978 of 40 
successful and 40 unsuccessful manufacturing companies whose shares were traded on the New York Stock Exchange (NYSE). It was determined that the current ratio is one of the determinants of financial failure in all models. Frydman et al. (1985) carried out financial failure modelling by using the data of 58 unsuccessful and 142 successful manufacturing companies listed in the USA during the 1971-1981 period, and 20 ratios including working capital ratio, current ratio and liquid assets ratio (cash stocks/total assets) were used. They determined that the financial ratio affects financial failure. Odom \& Sharda (1990) included 64 bankrupt and 65 non-bankrupt companies listed in the USA during the 1975-1982 periods in their study. Tirapat \& Nittayagasetwat (1999) studied 55 unsuccessful, and 341 successful companies listed in Thailand in 1997; Jones \& Hensher (2004) studied 24 unsuccessful and 62 successful IT and service companies listed in Australia in the period 1999-2003, whilst Salehi \& Abedini (2009) used data from 30 successful and 30 unsuccessful companies listed in Iran between 1995-2007. As a result of all four studies, it has been found that the working capital ratio is one of the determinants of financial failure.

Ganesalingam \& Kumar (2001) used the data of 42 successful and 29 unsuccessful companies listed in Australia between 1986 and 1998 to make financial failure predictions. As a result of the study, it has been determined that ten financial ratios, including the current ratio, acid-test ratio and cash ratio, can be used in estimating financial failure. In addition, Gruszczynski (2004), in his study on 200 companies listed in Poland between 1995-1997, and Keener (2013) in his study on 1203 retail companies listed in the USA between 2005 and 2013, found that the cash ratio had statistically significant effects on the financial failures of firms.

Chen et al. (2006) analyzed 89 unsuccessful and 940 successful companies listed in China between December 1999 and June 2003, and Ijaz et al. (2013) investigated the financial ratios affecting financial failure by using the financial data of 35 sugar companies in Pakistan between 2009 and 2010. As a result of both studies, it was determined that the current ratio has statistically significant effects on financial failure and can be used in financial failure predictions. Almansour (2015), on the other hand, examined the internal determinants of financial failure by using data from 11 successful and 11 unsuccessful companies listed in Jordan during the 2000-2003 period and found that the working capital ratio and current ratio had statistically significant effects on financial failure.

Tian \& Yu (2017) used 29 financial ratios of 108 Japanese and 112 European companies listed in Japan and European countries in the 1998-2012 period. Different financial failure models for Japanese and European companies were developed, and the results of their models were compared to the results of Altman's (1968) Z score model. As a result of the study, it was stated that retained earnings/total assets, leverage ratio and short-term liabilities/sales ratios were chosen for all models created for Japan, and equity/total debt ratio for European countries. In addition, it was noted that their model performed better than the Altman (1968) Z score model.

Fejér-Király et al. (2019) analyzed the determinants of financial failure by using data from 65 unsuccessful and 95 successful companies traded in the Bucharest Stock Exchange. As a result of the PAGE 86| Journal of Corporate Governance, Insurance, and Risk Management | 2021, VOL. 8, Series. 2 
study, it was determined that the variable of net working capital was selected for the model, which was developed to predict financial failure 1 and 2 years before, and it had significant effects on financial failure. Li \& Faff (2019) developed a model using the data of 421 unsuccessful and 441 successful companies between 1988 and 2011, and it was noted that the working capital ratio is one of the 11 variables that affect financial failure.

One of the first financial failure modelling studies in Turkey was carried out by Göktan in 1981. Göktan (1981) predicted the financial failures of the companies 1, 2, 3 and 4 years before the failure, based on the data of 25 successful and 14 unsuccessful companies between 1976 and 1980. As a result of the study, nine financial ratios, including the current ratio, were included in the developed model. One of the first studies to make financial failure predictions with multidimensional statistical models in Turkey was carried out by Aktaş in 1993. Aktaş (1993) developed a financial failure prediction model based on data from 25 successful and 35 unsuccessful companies between 1980 and 1989. As a result of the study, it was determined that the current ratio, acid-test ratio and liquid assets ratio, and working capital management were among the determinants of financial failure. Ünsal (2001) examined the financial ratios that can predict financial failure using data from 16 unsuccessful and 55 successful companies and stated that cash ratio, acid-test ratio and current ratio are among the variables that are determinants of financial failure. Aktaş et al. (2003) developed a financial failure prediction model using the data from 53 successful and 53 unsuccessful industrial, commercial and service companies between 1983 and 1997. As a result of the study, five financial ratios, including the acid-test ratio, were determined. It has also been determined that some influential variables can be used to predict financial failure. In the study of İçerli \& Akkaya (2006), 40 unsuccessful and 40 successful companies operating in the 1990-2003 period were examined with financial ratios and the Z test. The cash, acid-test, and current ratios were significant predictors of financial failure. Terzi (2011), on the other hand, aimed to develop a model to predict the financial failure risks of companies based on the data of 10 unsuccessful and 12 successful companies between 2009 and 2010. As a result of the study, six among nineteen financial ratios, including the net working capital ratio, were determined to predict failure effectively. Zeytinoğlu \& Akarim (2013) developed a year-specific financial failure model for 2009, 2010 and 2011 with 115 companies. They found that the current ratio was dominant only in the 2009 model, and the net working capital ratio was the main factor of financial failure in all three models.

Ural et al. (2015) analyzed the financial failure risks of companies for one, two and three years before the failure, using the data of 24 food, beverage and tobacco companies in the 2005-2012 period. They used five financial ratios without any working capital variables for one year before failure, seven financial ratios including current ratio and acid-test ratio for two years before financial failure, and eight financial ratios including cash ratio and inventory ratio for three years before financial failure. They determined the current ratio as the significant variable of the financial failure prediction models. Toraman \& Karaca (2016) used the data of 17 chemical companies between 2010 and 2013 and PAGE 87| Journal of Corporate Governance, Insurance, and Risk Management | 2021, VOL. 8, Series. 2 
examined the effects of various financial ratios with the Altman (1968) $\mathrm{Z}$ score values. They found that the working capital ratio has a significant effect on the Z-score values of the companies. Akyüz et al. (2017) examined sixteen paper and paper products companies operating in 2015. Ertan \& Ersan (2018) investigated financial failure using ratios of 175 successful and 33 unsuccessful manufacturing companies between 2000 and 2004. Karadeniz \& Öcek (2019) examined the financial ratios affecting financial failure by using the financial data of 12 tourism companies operating in the period between 2012 and 2017. As a result of all three studies, it has been determined that cash ratio, acid-test ratio, and current ratio had statistically significant effects on financial failure and can be used in financial failure predictions. Karaca and Özen (2017) investigated financial failures in the tourism sector companies in Borsa İstanbul using the Altman model.

Arslantürk Çöllü et al. (2020) determined the financial ratios affecting financial failure by using 20162018 data of textile, clothing and leather companies whose shares are listed in the BIST. They found that the current ratio, trade receivables ratio and inventory turnover are among the financial ratios that affect financial failure. In the study by Temelli \& Tekin (2020), 241 companies in Borsa Istanbul between 2011 and 2019 were analyzed with the Springate model. It was found that $77.6 \%$ of the companies were financially successful in the analyzed period.

\section{SCOPE, DATA AND METHODOLOGY}

Manufacturing industry companies listed on BIST between 2019 and 2020 are within the scope of this research. Data and information of BIST manufacturing industry companies were obtained from the Public Disclosure Platform (KAP). As of July 2021, there are 180 companies operating in the BIST manufacturing industry (KAP, 2021). The 146 manufacturing industry companies analyzed in this study are given in Table 1.

Table 1: BIST Manufacturing Companies Analyzed Within the Scope of the Research

\begin{tabular}{|c|c|c|}
\hline No. & Company & Code \\
\hline 1 & Acıselsan Acıpayam Selüloz Sanayi Tic. A.Ş. & ACSEL \\
\hline 2 & Adel Kalemcilik Tic.ve San. A.Ș. & ADEL \\
\hline 3 & Afyon Çimento Sanayi T.A.Ș. & AFYON \\
\hline 4 & Akçansa Çimento Sanayi ve Tic. A.Ș. & AKCNS \\
\hline 5 & Akın Tekstil A.Ș. & ATEKS \\
\hline 6 & Aksa Akrilik Kimya Sanayi A.Ș. & AKSA \\
\hline 7 & Alarko Carrier Sanayi ve Tic. A.Ș. & ALCAR \\
\hline 8 & Alcatel Lucent Teletaş Telekomünikasyon A.Ş. & ALKA \\
\hline 9 & Alkim Kağıt Sanayi ve Tic.A.Ş. & ALKİM \\
\hline 10 & Anadolu Efes Biracıllk ve Malt Sanayii A.Ș. & AEFES \\
\hline 11 & Arçelik A.Ş. & ARCLK \\
\hline 12 & Arsan Tekstil Ticaret ve Sanayi A.S.. & ARSAN \\
\hline 13 & Anadodu Isuzu Otomotiv Sanayi ve Ticaret A.Ș. & ASUZU \\
\hline 14 & A.V.O.D.Kurutulmuş Gida ve Tarım Ürünleri San. Tic. A.Ş. & AVOD \\
\hline 15 & Aygaz A.Ș. & AYGAZ \\
\hline 16 & Bağfaş Bandırma Gübre Fabrikaları A.Ș. & BAGFS \\
\hline 17 & Bak Ambalaj San.ve Tic. A.Ș. & BAKAB \\
\hline 18 & Banvit Bandırma Vitaminli Yem San. A.Ș. & BANVT \\
\hline 19 & Berkosan Yalıtım ve Tecrit Mad. Üretim ve Tic. A.Ş. & BRKSN \\
\hline 20 & Bilici Yatırım Sanayi ve Tic. A.Ș. & BLCYT \\
\hline 21 & Bantaș Bandırma Ambalaj San. Tíc. A.Ș. & BNTAS \\
\hline 22 & Batı Söke Söke Çimento San. T.A.Ş. & BSOKE \\
\hline 23 & Batıçim Batı Anadolu Çimento San. A.Ş. & BTCIM \\
\hline 24 & Birko Birleşik Koyunlular Mensucat Tic.ve San. A.Ș. & BRKO \\
\hline 25 & Birlik Mensucat Tic.ve San. İșletmesi A.Ș. & BRMEN \\
\hline 26 & Borusan Mannesmann Boru San.ve Tic. A.Ș. & BRSAN \\
\hline 27 & Bossa Tic. Ve San İșletmeleri T.A.Ș. & BOSSA \\
\hline 28 & Bosch Fren Sistemleri San.ve Tic. A.Ș. & BFREN \\
\hline 29 & Brisa Bridgestone Sabanc1 Lastik San.ve Tic. A.Ş. & BRİSA \\
\hline 30 & Burçelik Bursa Çelik Döküm Sanayi A.Ș. & BURCE \\
\hline 31 & Burçelik Vana San.ve Tic. A.Ș. & BURVA \\
\hline
\end{tabular}

PAGE 88| Journal of Corporate Governance, Insurance, and Risk Management | 2021, VOL. 8, Series. 2 


\begin{tabular}{|c|c|c|}
\hline 32 & Bursa Çimento Fabrikası A.Ş. & BUCiM \\
\hline 33 & Coca-Cola İçecek A.Ș. & CCOLA \\
\hline 34 & Çelik Halat ve Tel San. A.Ș. & CELHA \\
\hline 35 & Çemaş Döküm Sanayi A.Ș. & CEMAS \\
\hline 36 & Çemtaş Çelik Makine Sanayi ve Tic. A.Ș. & CEMTS \\
\hline 37 & Çimbeton Hazır beton ve Prefabrik Yapı Elemanları San. Ve Tic. A.Ș. & CMBTN \\
\hline 38 & Çimentaş İzmir Çimento Fabrikası T.A.Ș. & CMENT \\
\hline 39 & Çimsa Çimento Sanayi Tic. A.Ș. & CIMSA \\
\hline 40 & Çuhadaroğlu Metal San. Ve Paz. A.Ş. & CUSAN \\
\hline 41 & Dagi Giyim Sanayi ve Tic. A.Ș. & DAGİ \\
\hline 42 & Dardanel Önentaş Gıda Sanayi A.Ș. & DARDL \\
\hline 43 & Demisaş Döküm Emaye Mamulleri San. A.Ș. & DMSAS \\
\hline 44 & Derimod Konf. Ayakkabı Deri San ve Tic. A.Ș. & DERIM \\
\hline 45 & Desa Deri San.ve Tic. A.Ș. & DESA \\
\hline 46 & Deva Holding A.Ş. & DEVA \\
\hline 47 & Diriteks Diriliş Tekstil San.ve Tic. A.Ş. & DIRIT \\
\hline 48 & Ditas Doğan Yedek Parça İmalat ve Teknik A.Ş. & DİTAS \\
\hline 49 & Doğan Burda Dergi Yayıncıllk ve Paz. A.Ș. & DOBUR \\
\hline 50 & Doğtaș Kelebek Mobilya San ve Tic. A.Ș. & DGKLB \\
\hline 51 & Doğusan Boru san ve Tic. A.Ș. & DOGUB \\
\hline 52 & Döktaş Dökümcülük Tc.ve San. A.Ş. & DOKTA \\
\hline 53 & Duran Doğan Basın ve Ambalaj San. A.Ş. & DURDO \\
\hline 54 & DYO Boya Fabrikaları San.ve Tic. A.Ș. & DYOBY \\
\hline 55 & Ege Endüstri ve Tic. A.Ș. & EGEEN \\
\hline 56 & Ege Gübre Sanayi A.Ş. & EGGUB \\
\hline 57 & Ege Profil Ticaret ve San. A.S.. & EGPRO \\
\hline 58 & Ege Seramik San.ve Tic. A.S.s. & EGESER \\
\hline 59 & Ege Plast Ege Plastik Tic.ve San A.Ș. & EPLAS \\
\hline 60 & Ekiz Kimya San.ve Tic. A.Ș. & EKİZ \\
\hline 61 & Emek Elektrik End. A.Ș. & EMKEL \\
\hline 62 & Eminiş Ambalaj San.ve Tic. A.Ș. & EMNIS \\
\hline 63 & Erbosan Erciyas Boru Sanayii Tic. A.Ș. & ERBOS \\
\hline 64 & Ereğli Demir Çelik Fabrikaları T.A.Ș. & EREGL \\
\hline 65 & Ersu Meyve ve Gida San. A.Ș. & ERSU \\
\hline 66 & Federal -Mogul İzmit Piston ve Pim Üretim Tesisleri A.Ş. & FMZIP \\
\hline 67 & Ford Otomotiv San .A.Ș. & FROTO \\
\hline 68 & Formet Çelik Kapı San. Ve Tic. A.Ş. & FORMT \\
\hline 69 & Frigo-Pak Gida Maddeleri San. ve Tic. A.Ș. & FRIGGO \\
\hline 70 & Gediz Ambalaj San.ve Tic. A.Ș. & GEDZA \\
\hline 71 & Gentaş Dekoratif Yüzeyler San.ve Tic. A.Ş. & GENTS \\
\hline 72 & Gersan Elektrik Tic. Ve San. A.Ş. & GEREL \\
\hline 73 & Goodyear Lastikleri T.A.Ș. & GOODY \\
\hline 74 & Göltaş Göller Bölgesi Çimento Sanayi Tic. A.Ș. & GOLTS \\
\hline 75 & Gübre Fabrikaları T.A.Ș. & GUBRF \\
\hline 76 & Hateks Hatay Tekstil İşletmeleri A.Ș. & HATEK \\
\hline 77 & Hektaş Tic. T.A.Ş. & HEKTS \\
\hline 78 & Hürriyet Gazetecilik ve Matbaacilık A.Ș. & HURGZ \\
\hline 79 & İhlas Ev Aletleri İmalat Sanayi ve Tic. A.Ș. & IHEVA \\
\hline 80 & İhlas Gazetecilik A.Ș. & IHGZT \\
\hline 81 & İskenderun Demir Çelik A.Ş. & ISDMR \\
\hline 82 & İzmir Demir Çelik San. A.Ș. & IZDMC \\
\hline 83 & Jantsa Jant Sanayi ve Tic. A.Ș. & JANTS \\
\hline 84 & Kaplamin Ambalaj Sanayi ve Tic. A.Ş. & KAPLM \\
\hline 85 & Kardemir Karabük Demir Çelik Sanayi Tic. A.Ş. & KARDMD \\
\hline 86 & Karsan Otomotiv Sanayii ve Tic. A.Ș & KARSN \\
\hline 87 & Karsu Tekstil Sanayii ve Tic. A.Ș & KRTEK \\
\hline 88 & Kartonsan Karton San. Ve Tic. A.Ș. & KARTN \\
\hline 89 & Katmerciler Araç Üstü Ekipman San. Ve Tic. A.Ș. & KATMR \\
\hline 90 & Kent Gida Maddeleri San. Ve Tic. A.Ș. & KENT \\
\hline 91 & Kerevitaş Gıda San. Ve Tic.A.Ş. & KERVT \\
\hline 92 & Klimasan Klima San. Ve Tic.A.Ş. & KLMSN \\
\hline 93 & Konfrut Gıda San. Ve Tic.A.Ș. & KONFRT \\
\hline 94 & Konya Çimento Sanayii A.Ș. & KONYA \\
\hline 95 & Kordsa Teknik Tekstil A.Ș. & KORDS \\
\hline 96 & Kristal Kola ve Meșrubat Sanayi Ticaret A.Ș. & KRSTL \\
\hline 97 & Kütahya Porselen Sanayi A.Ș. & KUTPO \\
\hline 98 & Lüks Kadife Ticaret ve Sanayii A.Ș. & LUKSK \\
\hline 99 & Makina Takım Endüstrisi A.Ș. & MAKTK \\
\hline 100 & Marshall Boya ve Vernik Sanayi A.Ș. & MRSHL \\
\hline 101 & Menderes Tekstil Sanayi ve Ticaret A.Ș. & MNDRS \\
\hline 102 & Mondi Olmuksan Kağıt ve Ambalaj Sanayi A.Ș. & OLMK \\
\hline 103 & Mondi Tire Kutsan Kağıt ve Ambalaj Sanayi A.Ș. & TIRE \\
\hline 104 & Niğbaş Niğde Beton Sanayi ve Ticaret A.Ș. & NIBAS \\
\hline 105 & Nuh Çimento Sanayi A.Ș. & NUHCM \\
\hline 106 & Otokar Otomotiv ve Savunma Sanayi A.Ș. & OTKAR \\
\hline 107 & Oylum Sinai Yatırımlar A.Ș. & OYLUM \\
\hline 108 & Özbal Çelik Boru Sanayi Tic. Ve Taahhüt A.Ș. & OZBAL \\
\hline 109 & Parsan Makine Parçaları Sanayii A.Ș. & PARSN \\
\hline 110 & Penguen Gida Sanayi A.Ș. & PENGD \\
\hline 111 & Petkim Petrokimya Holding A.Ș. & PETKM \\
\hline 112 & Pinar Entegre Et ve Un Sanayii A.Ş. & PETUN \\
\hline 113 & Pınar Su ve İçecek Sanayi ve Ticaret A.Ș. & PINSU \\
\hline 114 & Pinar Süt Mamulleri Sanayii A.Ș. & PNSUT \\
\hline 115 & Prizma Pres Matbaacılık Yayıncılık Sanayi ve Tic. A.Ș. & PRZMA \\
\hline 116 & Royal Halı İplik Tekstil Mobilya Sanayi ve Tic. A.Ș. & ROYAL \\
\hline 117 & RTA Laboratuvarları Biyolojik Ürünler İlaç ve Makine San. Tic. A.Ș. & RTLAB \\
\hline 118 & Sanifoam Sünger Sanayi ve Ticaret A.Ș. & SANFM \\
\hline 119 & Saray Matbaacılık Kağıtçılık Kırtasiyecilik Tic. Ve San. A.Ș. & SAMAT \\
\hline 120 & Sarkuysan Elektrolikit Bakır Sanayi ve Tic.A.Ș. & SARKY \\
\hline 121 & Say Yenilenebilir Enerji Ekipmanları Sanayi ve Tic. A.Ș. & SAYAS \\
\hline 122 & Sasa Polyester Sanayi A.Ş. & SASA \\
\hline 123 & Sekuro Plastik Ambalaj Sanayi A.Ș. & SEKUR \\
\hline 124 & Selçuk Gıda Endüstri İhracat ve İthalat A.Ş. & SELGD \\
\hline 125 & Silverline Endüstri ve Ticaret A.Ș. & SILVR \\
\hline
\end{tabular}

PAGE 89| Journal of Corporate Governance, Insurance, and Risk Management | 2021, VOL. 8, Series. 2 


\begin{tabular}{|c|c|c|}
\hline 126 & Söktaş Tekstil Sanayi ve Tic.A.Ş. & SKTAS \\
\hline 127 & Sönmez Pamuklu Sanayii A.Ș. & SNPAM \\
\hline 128 & Tat Gıda Sanayi A.S.S. & TATGD \\
\hline 129 & Temapol Polimer Plastik ve İnşaat Sanayi Ticaret A.Ș. & TMPOL \\
\hline 130 & Tofaș Türk Otomobil Fabrikası A.Ș. & TOASO \\
\hline 131 & Tuğçelik Alüminyum ve Metal Mamulleri Sanayi ve Tic.A.Ș. & TUCLK \\
\hline 132 & Tukaş Gıda Sanayi ve Tic.A.Ș. & TUKAS \\
\hline 133 & Tümosan Motor ve Traktör Sanayi A.Ş. & TMSN \\
\hline 134 & Tüpraş-Türkiye Petrol Rafinerileri A.Ş. & TUPRS \\
\hline 135 & Türk Prysmian Kablo Sistemleri A.Ș. & PRKAB \\
\hline 136 & Türk Traktör ve Ziraat Makineleri A.Ș. & TTRAK \\
\hline 137 & Türk Tuborg Bira ve Malt Sanayii A.Ș. & TBORG \\
\hline 138 & Ulusoy Elektrik İmalat Taahhüt ve Tic.A.Ș. & ULUSE \\
\hline 139 & Ulusoy Un Sanayi ve Ticaret A.Ș. & ULUUN \\
\hline 140 & Ușak Seramik Sanayi A.Ș. & USAK \\
\hline 141 & Ülker Bisküvi Sanayi A.Ș. & ULKER \\
\hline 142 & Vestel Beyaz Eşya Sanayi ve Tic. A.Ş. & VESBE \\
\hline 143 & Vestel Elektronik Sanayi ve Ticaret A.Ş. & VESTL \\
\hline 144 & Viking Kağıt ve Selüloz A.Ș. & VKING \\
\hline 145 & Yataş Yatak ve Yorgan Sanayi ve Ticaret A.Ș. & YATAS \\
\hline 146 & Yünsa Yünlü Sanayi ve Ticaret A.Ș. & YUNSA \\
\hline
\end{tabular}

Source: Authors' Compilation

While Mondi Olmuksan Kağıt ve Ambalaj Sanayi A.Ş was traded on the BIST with the OLMIP code, it started to be traded with the OLMK code on 01.07.2021 due to the company name change and was included in the analysis with the same code. Of the 34 manufacturing industry companies not included in the research, 11 were offered to the public (IPOs) in 2021, 3 in 2020 and 1 in 2019 and started to be traded on the BIST. They were not included in the analysis due to the lack of financial statements for the periods studied. Merko Gida Sanayi ve Tic. A.Ş. (MERKO) was excluded from the analysis. Oyak Çimento (OYAKC) was excluded from the scope of the analysis since Oyak Cement group companies were merged under Mardin Çimento in 2019, and the name of Mardin Çimento was changed to Oyak Çimento since it does not have financial statements for the relevant year. Seventeen manufacturing companies that publish their financial reports every six months are also excluded from the analysis. The companies not included in the study are given in Table 2.

The research used financial statement data of BIST manufacturing companies published in quarterly periods between 2019 and 2020. The companies' financial statement data within the research scope for the relevant periods were taken from the KAP and Fintables databases. In addition, the closing prices of stocks are taken from investing.com.

Within the scope of the research, the quarterly financial statements of 146 BIST companies operating in the manufacturing industry sector for the years 2019-2020 were evaluated using Altman (1968), Springate (1978), Taffler (1983) and Zmijewski (1984) financial failure models. The financial failure models and calculations used in the study are given in Table 3.

Table 2: BIST Manufacturing Companies Excluded from the Scope of the Research

\begin{tabular}{|c|c|c|}
\hline No. & Company & Code \\
\hline & IPOs in 2021 & \\
\hline 1 & BMS Çelik Hasır Sanayi ve Ticaret A.Ș. & BMS \\
\hline 2 & Kalekim Kimyevi Maddeler San. ve Tic.A.Ş. & KALKIM \\
\hline 3 & Işık Plastik Sanayi ve Dış Ticaret Pazarlama A.Ș. & ISKPL \\
\hline 4 & Kervan Gıda Sanayi ve Ticaret A.Ș. & KRVGD \\
\hline 5 & Kütahya Şeker Fabrikası A.Ș. & KTSKR \\
\hline 6 & Meditera Tibbi Malzeme Sanayi ve Ticaret A.Ș. & MEDTR \\
\hline 7 & Mercan Kimya Sanayi ve Ticaret A.Ș. & MERCN \\
\hline 8 & Qua Granite Hayal Yapı ve Ürünleri Sanayi Ticaret A.Ș. & QUAGR \\
\hline 9 & Selva Gıda Sanayi A.Ş. & SELVA \\
\hline 10 & Türk İlaç ve Serum Sanayi A.Ș. & TRILC \\
\hline \multirow[t]{2}{*}{11} & Boğaziçi Beton Sanayi Tic.A.Ș. & BOBET \\
\hline & IPOs in 2020 & \\
\hline 1 & Fade Gida Yatırım ve Sanayi Ticaret A.Ș. & FADE \\
\hline 2 & Bayrak EBT Taban Sanayi ve Ticaret A.S. & BAYRK \\
\hline 3 & Dinamik Is1 Makine Yalıtım Malzemeleri Sanayi ve Ticaret_A.Ș. & DNISI \\
\hline
\end{tabular}

PAGE 90| Journal of Corporate Governance, Insurance, and Risk Management | 2021, VOL. 8, Series. 2 


\begin{tabular}{|c|c|c|}
\hline & IPOs in 2019 & \\
\hline \multirow[t]{2}{*}{1} & Yükselen Çelik A.Ș. & YKSLN \\
\hline & Companies that publish Financial Reports on a 6-monthly basis & \\
\hline 1 & Ayes Çelik Hasır ve Çit Sanayi A.S.S. & AYES \\
\hline 2 & Balatacılar Balatacılık Sanayi ve Ticaret A.Ș. & BALAT \\
\hline 3 & Baștaş Başkent Çimento Sanayi ve Ticaret A.Ș. & BASCM \\
\hline 4 & İzmir Fırça Sanayi ve Ticaret A.Ș. & İZFAS \\
\hline 5 & Mega Polietilen Köpük Sanayi ve Ticaret A.Ş. & MEGAP \\
\hline 6 & Orma Orman Mahsulleri İntegre Sanayi ve Ticaret A.Ș. & ORMA \\
\hline 7 & Özerden Plastik ve Sanayi Ticaret A.Ş. & OZRDN \\
\hline 8 & Rodrigo Tekstil ve Sanayi Ticaret A.Ș. & RODRG \\
\hline 9 & Politeknik Metal Sanayi ve Ticaret A.Ș. & POLTEK \\
\hline 10 & Sodaş Sodyum Sanayii A.Ş. & SODSN \\
\hline 11 & Taze Kuru Gıda Sanayi ve Ticaret A.Ș. & TKURU \\
\hline 12 & Sumaș Suni Tahta ve Mobilya Sanayi A.Ș. & SUMAS \\
\hline 13 & Vanet Gıda Sanayi İç ve Dış Ticaret A.Ș. & VANGD \\
\hline 14 & Yibitaş Yozgat İşçi Birliği İnşaat Malzemeleri Ticaret ve San. A.Ş. & YİBITAȘ \\
\hline 15 & Yonga Mobilya Sanayi ve Ticaret A.Ș. & YONGA \\
\hline 16 & Safkar Ege Soğutmacilık Klima Soğuk Hva Tes. İhracat İthalat San. A.Ş. & SAFKAR \\
\hline 17 & Seyitler Kimya Sanayi A.Ș. & SEYKM \\
\hline
\end{tabular}

Source: Authors' Compilation

Table 3: Financial Failure Models in the Research

\begin{tabular}{|c|c|c|}
\hline $\begin{array}{c}\text { Financial } \\
\text { Failure } \\
\text { Model }\end{array}$ & Formula & Result \\
\hline $\begin{array}{c}\text { Altman } \\
(1968)\end{array}$ & 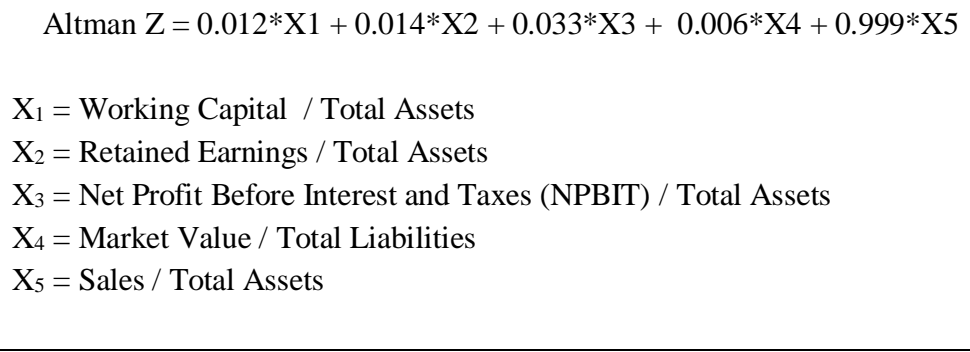 & $\begin{array}{l}\mathrm{Z}<1,81 \text { then the company } \\
\text { is classified as "not at risk } \\
\text { of financial failure" } \\
1,81 \leq \mathrm{Z} \leq 2,99 \text { then the } \\
\text { company is classified as } \\
\text { "uncertain" } \\
\mathrm{Z}>2,99 \text { then the company } \\
\text { is classified as "not at risk } \\
\text { of financial failure" }\end{array}$ \\
\hline $\begin{array}{c}\text { Springate } \\
\text { (1978) }\end{array}$ & $\begin{array}{l}\qquad \text { Springate }_{\mathrm{z}}=1.03 * \mathrm{X}_{1}+3.07 * \mathrm{X}_{2}+0.66 * \mathrm{X}_{3}+0.4 * \mathrm{X}_{4} \\
\mathrm{X} 1 \text { = Working Capital } / \text { Total Assets } \\
\mathrm{X} 2=\text { Net Profit Before Interest and Taxes (NPBIT) } / \text { Total Assets } \\
\mathrm{X} 3=\text { Net Profit Before Taxes (NPAT) } / \text { Current Liabilities } \\
\mathrm{X} 4=\text { Sales } / \text { Total Assets }\end{array}$ & $\begin{array}{l}\mathrm{Z}<0.862 ; \text { then the } \\
\text { company is classified as } \\
\text { "at risk of financial } \\
\text { failure" } \\
\mathrm{Z}>0.862 \text {; then the } \\
\text { company is classified as } \\
\text { "not at risk of financial } \\
\text { failure" }\end{array}$ \\
\hline $\begin{array}{l}\text { Taffler } \\
(1983)\end{array}$ & $\begin{array}{l}\quad \text { Tafflerz }=3.20+12.18 * \mathrm{X}_{1}+2.50 * \mathrm{X}_{2}-10.68 * \mathrm{X}_{3}+0.03 * \mathrm{X}_{4} \\
\mathrm{X}_{1}=\text { Net Profit Before Taxes }(\mathrm{NPAT}) / \text { Average Short Term Liabilities } \\
\mathrm{X}_{2}=\text { Current Assets } / \text { Total Liabilities } \\
\mathrm{X}_{3}=\text { Short Term Liabilities } / \text { Total Assets } \\
\mathrm{X}_{4}=(\text { Current Assets }- \text { Stoklar }- \text { Short Term Liabilities }) /(\text { Sales }- \text { Net Profit } \\
\text { Before Taxes + Amortization })\end{array}$ & $\begin{array}{l}\mathrm{Z}<0,3 \text { then the company } \\
\text { is classified as "at risk of } \\
\text { financial failure", } \\
\mathrm{Z}>0,3 \text { then the company } \\
\text { is classified as "not at risk } \\
\text { of financial failure" }\end{array}$ \\
\hline $\begin{array}{c}\text { Zmijewski } \\
\text { (1984) }\end{array}$ & $\begin{array}{l}\quad \text { Zmijewski }=-4.336-4.513 * \mathrm{X} 1+5.769 * \mathrm{X} 2+0.004 * \mathrm{X} 3 \\
\mathrm{X} 1=\text { Net Income } / \text { Total Assets }=\text { ROA } \\
\mathrm{X} 2=\text { Total Liabilities } / \text { Total Assets }=\text { Leverage } \\
\mathrm{X} 3 \text { = Current Assets } / \text { Current Liabilities }=\text { Current Ratio = Liquidity }\end{array}$ & $\begin{array}{l}Z>0 \text { then the company is } \\
\text { classified as "at risk of } \\
\text { financial failure", } \\
Z<0 \text { then the company is } \\
\text { classified as "not at risk of } \\
\text { financial failure" }\end{array}$ \\
\hline
\end{tabular}

\section{Source: Authors' Compilation}

PAGE 91| Journal of Corporate Governance, Insurance, and Risk Management | 2021, VOL. 8, Series. 2 
Abbreviations are used in the tables while giving the financial status of the companies. RoFF (Risk of Financial Failure) was used for companies with a risk of financial failure, NRoFF (No Risk of Financial Failure) for companies with no risk of financial failure, and UN (Uncertain) for companies with an uncertain risk of financial failure.

\section{FINDINGS}

The Altman $\mathrm{Z}$ values of the BIST manufacturing companies within the scope of the research, calculated between 2019 and 2020, are given in Table 4.

Table 4: Altman Z Values of Manufacturing Companies for 2019-2020

\begin{tabular}{|c|c|c|c|c|c|c|c|c|c|}
\hline \multirow{2}{*}{ No. } & \multirow{2}{*}{ Code } & \multicolumn{8}{|c|}{ Altman Z } \\
\hline & & $2019 / 3$ & $2020 / 3$ & $2019 / 6$ & $2020 / 6$ & $2019 / 9$ & $2020 / 9$ & $2019 / 12$ & $2020 / 12$ \\
\hline 1 & ACSEL & 4,83 & 5,52 & 4,50 & 9,03 & 5,63 & 20,10 & 6,46 & 13,08 \\
\hline 2 & ADEL & 1,40 & 1,02 & 1,03 & 1,07 & 1,09 & 1,15 & 1,72 & 1,49 \\
\hline 3 & AFYON & 0,13 & $-0,07$ & 0,09 & 4,15 & 0,18 & 5,48 & 0,15 & 5,89 \\
\hline 4 & AKCNS & 0,92 & 0,96 & 1,00 & 1,75 & 1,16 & 1,92 & 1,61 & 2,04 \\
\hline 5 & ATEKS & 1,25 & 1,13 & 1,28 & 1,41 & 1,27 & 1,88 & 1,46 & 2,76 \\
\hline 6 & AKSA & 0,84 & 1,01 & 0,74 & 0,83 & 0,74 & 1,14 & 0,75 & 1,71 \\
\hline 7 & ALCAR & 3,42 & 2,90 & 2,78 & 3,55 & 3,37 & 5,08 & 3,96 & 10,51 \\
\hline 8 & ALKA & 1,64 & 3,01 & 2,48 & 6,46 & 2,39 & 6,48 & 3,49 & 6,30 \\
\hline 9 & ALKİM & 1,90 & 2,54 & 2,03 & 6,71 & 1,95 & 6,30 & 2,25 & 7,28 \\
\hline 10 & AEFES & 0,52 & 0,56 & 0,66 & 0,69 & 0,74 & 0,72 & 0,67 & 0,67 \\
\hline 11 & ARCLK & 1,06 & 1,01 & 1,09 & 1,12 & 1,10 & 1,09 & 1,09 & 1,15 \\
\hline 12 & ARSAN & 0,84 & 1,25 & 0,96 & 1,35 & 1,04 & 1,84 & 0,93 & 2,17 \\
\hline 13 & ASUZU & 0,50 & 0,61 & 0,45 & 0,82 & 0,50 & 0,83 & 1,00 & 1,16 \\
\hline 14 & AVOD & 1,08 & 0,99 & 1,13 & 1,43 & 0,97 & 2,06 & 0,81 & 2,37 \\
\hline 15 & AYGAZ & 1,46 & 1,53 & 1,68 & 1,70 & 1,80 & 1,74 & 1,94 & 1,96 \\
\hline 16 & BAGFS & 0,65 & 0,76 & 0,57 & 0,78 & 0,75 & 0,78 & 0,71 & 0,87 \\
\hline 17 & BAKAB & 1,19 & 1,42 & 1,15 & 1,44 & 1,26 & 1,75 & 1,39 & 2,21 \\
\hline 18 & BANVT & 1,58 & 1,68 & 2,21 & 2,02 & 1,97 & 1,96 & 2,01 & 2,67 \\
\hline 19 & BRKSN & 0,86 & 0,94 & 0,74 & 1,35 & 0,84 & 2,06 & 1,06 & 2,64 \\
\hline 20 & BLCYT & 1,09 & 3,49 & 1,13 & 3,30 & 1,63 & 6,27 & 2,66 & 6,39 \\
\hline 21 & BNTAS & 2,99 & 5,08 & 2,81 & 6,00 & 3,30 & 5,86 & 4,73 & 6,21 \\
\hline 22 & BSOKE & 0,12 & $-0,20$ & 0,01 & $-0,11$ & 0,06 & $-0,11$ & 0,01 & $-0,09$ \\
\hline 23 & BTCIM & 0,30 & 0,12 & 0,23 & 0,15 & 0,24 & 0,18 & 0,26 & 0,15 \\
\hline 24 & BRKO & $-0,98$ & $-1,14$ & $-0,89$ & $-1,17$ & $-1,04$ & $-0,06$ & $-1,11$ & 0,30 \\
\hline 25 & BRMEN & $-1,13$ & $-3,86$ & $-1,19$ & 0,50 & $-1,22$ & $-1,86$ & $-1,10$ & $-9,77$ \\
\hline 26 & BRSAN & 0,52 & 0,39 & 0,55 & 0,40 & 0,50 & 0,44 & 0,51 & 0,77 \\
\hline 27 & BOSSA & 0,71 & 0,95 & 0,81 & 0,97 & 0,81 & 1,35 & 1,10 & 1,70 \\
\hline 28 & BFREN & 7,84 & 9,51 & 7,13 & 10,39 & 9,64 & 27,45 & 11,54 & 27,83 \\
\hline 29 & BRISA & 0,64 & 0,52 & 0,61 & 0,68 & 0,69 & 0,84 & 0,66 & 1,38 \\
\hline 30 & BURCE & 0,15 & 0,23 & 0,14 & 0,41 & 0,22 & 1,55 & 0,43 & 1,29 \\
\hline 31 & BURVA & 0,89 & 4,14 & 0,92 & 4,78 & 2,27 & 11,30 & 4,20 & 16,52 \\
\hline 32 & BUCİM & 1,73 & 1,92 & 1,76 & 1,89 & 1,71 & 2,13 & 1,81 & 3,21 \\
\hline 33 & CCOLA & 0,98 & 1,08 & 1,08 & 1,29 & 1,21 & 1,40 & 1,10 & 1,48 \\
\hline 34 & CELHA & 0,49 & 0,38 & 0,57 & 0,24 & 0,44 & 0,54 & 0,32 & 0,75 \\
\hline 35 & CEMAS & 0,75 & 2,16 & 0,72 & 4,49 & 0,87 & 4,50 & 0,38 & 3,98 \\
\hline 36 & CEMTS & 4,54 & 5,37 & 5,47 & 8,42 & 5,87 & 7,82 & 7,86 & 11,10 \\
\hline 37 & CMBTN & 1,04 & 0,32 & 0,86 & 0,64 & 0,92 & 1,16 & 0,95 & 2,31 \\
\hline 38 & CMENT & 2,76 & 1,45 & 2,65 & 2,46 & 2,05 & 3,34 & 2,33 & 3,70 \\
\hline 39 & CIMSA & 0,64 & 0,54 & 0,59 & 0,82 & 0,71 & 0,92 & 0,75 & 0,75 \\
\hline 40 & CUSAN & 1,05 & 1,60 & 1,27 & 1,87 & 1,47 & 2,18 & 1,73 & 2,46 \\
\hline 41 & DAGİ & 0,34 & 0,38 & 0,49 & 0,85 & 0,53 & 0,56 & 1,37 & 0,84 \\
\hline 42 & DARDL & $-5,66$ & $-0,68$ & $-5,64$ & 0,66 & $-5,03$ & 0,92 & $-2,46$ & 1,48 \\
\hline 43 & DMSAS & 1,36 & 1,25 & 1,14 & 1,49 & 1,07 & 1,82 & 1,39 & 1,94 \\
\hline 44 & DERİM & 0,89 & 1,06 & 0,80 & 1,09 & 0,83 & 1,23 & 1,36 & 1,31 \\
\hline 45 & DESA & 0,52 & 0,72 & 0,70 & 0,43 & 0,66 & 1,36 & 0,96 & 1,72 \\
\hline 46 & DEVA & 1,36 & 2,13 & 1,46 & 2,77 & 1,57 & 2,81 & 1,84 & 2,98 \\
\hline 47 & DIRIT & $-1,19$ & $-1,30$ & $-1,91$ & $-1,00$ & $-2,30$ & $-1,37$ & $-2,34$ & $-1,12$ \\
\hline 48 & DİTAS & 0,80 & 0,74 & 0,82 & 0,89 & 0,75 & 1,70 & 0,90 & 1,62 \\
\hline 49 & DOBUR & 1,73 & 1,79 & 1,86 & 5,06 & 1,78 & 7,11 & 2,79 & 9,06 \\
\hline 50 & DGKLB & 0,03 & $-0,04$ & 0,13 & 0,21 & $-0,03$ & 0,39 & 0,07 & 0,40 \\
\hline 51 & DOGUB & $-1,00$ & 2,87 & 3,61 & 5,31 & 5,84 & 6,91 & 5,98 & 6,69 \\
\hline 52 & DOKTA & 0,46 & 0,89 & 0,56 & 1,46 & 1,04 & 1,44 & 1,46 & 1,64 \\
\hline 53 & DURDO & 0,46 & 0,80 & 0,46 & 0,68 & 0,73 & 0,77 & 0,81 & 1,07 \\
\hline 54 & DYOBY & 0,27 & 0,28 & $\begin{array}{l}0,31 \\
0,31\end{array}$ & 0,54 & 0,32 & 0,84 & $\begin{array}{l}, 019 \\
0,49\end{array}$ & 1,18 \\
\hline 55 & EGEEN & 5,07 & 4,38 & 4,68 & 5,11 & 4,84 & 5,43 & 5,61 & 10,92 \\
\hline 56 & EGGUB & 1,63 & 1,13 & 1,51 & 1,71 & 2,02 & 2,69 & 1,16 & 2,94 \\
\hline 57 & EGPRO & 1,24 & 1,28 & 1,17 & 1,40 & 1,31 & 1,73 & 1,44 & 2,76 \\
\hline 58 & EGESER & 2,19 & 1,75 & 2,00 & 2,00 & 1,86 & 2,31 & 2,10 & 3,04 \\
\hline 59 & EPLAS & 1,14 & 2,99 & 1,28 & 3,41 & 1,62 & 3,47 & 2,27 & 4,38 \\
\hline 60 & EKİZ & $-1,27$ & $-0,65$ & $-1,08$ & 0,06 & $-0,78$ & 0,08 & $-0,61$ & 0,25 \\
\hline 61 & EMKEL & $-0,11$ & 0,00 & 0,05 & 0,21 & $-0,03$ & 0,06 & 0,05 & 0,32 \\
\hline 62 & EMNIS & $-1,90$ & $-1,82$ & $-0,72$ & $-1,40$ & $-1,45$ & 0,68 & $-1,29$ & 2,37 \\
\hline 63 & ERBOS & 2,90 & 2,55 & 2,49 & 3,24 & 2,81 & 4,06 & 2,36 & 4,49 \\
\hline 64 & EREGL & 1,42 & 1,50 & 1,74 & 1,54 & 1,55 & 1,57 & 1,67 & 2,68 \\
\hline 65 & ERSU & 0,88 & 5,76 & 0,64 & 8,24 & 1,40 & 9,31 & 2,44 & 7,08 \\
\hline 66 & FMZiP & 13,46 & 30,43 & 17,89 & 59,38 & 16,78 & 78,11 & 41,59 & 77,56 \\
\hline 67 & FROTO & 1,76 & 1,90 & 2,01 & 1,83 & 2,20 & 2,22 & 2,36 & 3,07 \\
\hline 68 & FORMT & 2,10 & 0,78 & 1,67 & 0,96 & 2,43 & 1,22 & 0,98 & 1,30 \\
\hline 69 & FRİGO & 0,77 & 1,20 & 0,89 & 1,98 & 0,83 & 4,84 & 1,05 & 2,09 \\
\hline 70 & GEDZA & 2,41 & 2,95 & 2,39 & 3,99 & 2,70 & 4,00 & 2,60 & 4,83 \\
\hline 71 & GENTS & 2,01 & 2,69 & 1,94 & 3,15 & 2,12 & 3,60 & 2,71 & 5,26 \\
\hline
\end{tabular}

PAGE 92| Journal of Corporate Governance, Insurance, and Risk Management | 2021, VOL. 8, Series. 2 


\begin{tabular}{|c|c|c|c|c|c|c|c|c|c|}
\hline 72 & GEREL & 1,24 & 1,25 & 1,28 & 2,44 & 1,36 & 2,42 & 1,52 & 2,17 \\
\hline 73 & GOODY & 1,41 & 1,51 & 1,42 & 2,48 & 1,41 & 2,70 & 2,69 & 3,02 \\
\hline 74 & GOLTS & 0,88 & 0,87 & 0,77 & 0,93 & 0,75 & 0,99 & 0,83 & 1,13 \\
\hline 75 & GUBRF & 0,49 & 1,31 & 0,35 & 2,04 & 0,53 & 2,14 & 0,58 & 5,56 \\
\hline 76 & HATEK & 0,65 & 0,89 & 0,51 & 1,10 & 0,63 & 1,36 & 1,14 & 1,62 \\
\hline 77 & HEKTS & 1,29 & 1,72 & 1,08 & 1,20 & 0,86 & 1,11 & 1,28 & 1,14 \\
\hline 78 & $\begin{array}{l}\text { HURGZ } \\
\end{array}$ & 0,92 & 0,82 & 0,73 & 1,22 & 0,75 & 2,12 & 1,41 & 1,71 \\
\hline 79 & IHEVA & 1,96 & 5,53 & 2,06 & 5,16 & 4,96 & 6,99 & 9,11 & 6,41 \\
\hline 80 & IHGZT & 1,16 & 3,26 & 1,53 & 2,49 & 1,86 & 6,68 & 4,58 & 4,68 \\
\hline 81 & ISDMR & 1,53 & 2,33 & 2,72 & 2,36 & 2,60 & 2,15 & 2,85 & 3,31 \\
\hline 82 & IZDMC & $-0,48$ & $-0,45$ & $-0,41$ & $-0,52$ & $-0,37$ & $-0,29$ & $-0,33$ & $-0,18$ \\
\hline 83 & JANTS & 1,63 & 1,51 & 1,69 & 1,75 & 1,26 & 2,37 & 1,67 & 3,03 \\
\hline 84 & KAPLM & 0,41 & 0,63 & 0,32 & 0,82 & 0,35 & 1,57 & 0,53 & 2,77 \\
\hline 85 & KARDMD & 0,82 & 0,53 & 0,84 & 0,66 & 0,73 & 0,70 & 0,68 & 1,12 \\
\hline 86 & KARSN & 0,05 & 0,36 & 0,22 & 0,89 & 0,06 & 1,15 & 0,54 & 1,19 \\
\hline 87 & KRTEK & 0,64 & 0,64 & 0,63 & 0,55 & 0,77 & 0,87 & 0,60 & 0,89 \\
\hline 88 & KARTN & 1,35 & 1,23 & 1,31 & 1,33 & 1,19 & 1,59 & 1,24 & 35,36 \\
\hline 89 & KATMR & 0,56 & 0,97 & 0,92 & 1,01 & 0,93 & 1,29 & 1,10 & 1,32 \\
\hline 90 & KENT & 1,71 & 1,86 & 1,67 & 2,98 & 1,60 & 102,88 & 1,99 & 100,83 \\
\hline 91 & KERVT & 1,17 & 1,85 & 1,31 & 1,97 & 1,40 & 2,07 & 1,68 & 2,00 \\
\hline 92 & KLMSN & 1,31 & 1,13 & 1,37 & 1,38 & 1,22 & 1,28 & 1,52 & 1,43 \\
\hline 93 & KONFRT & 1,59 & 1,80 & 1,93 & 2,39 & 3,21 & 2,69 & 1,64 & 2,08 \\
\hline 94 & KONYA & 8,21 & 7,02 & 8,06 & 8,66 & 10,05 & 16,36 & 11,03 & 27,95 \\
\hline 95 & KORDS & 1,07 & 0,74 & 1,05 & 0,53 & 0,88 & 0,66 & 0,82 & 0,91 \\
\hline 96 & KRSTL & 3,30 & 5,73 & 3,98 & 10,49 & 5,06 & 10,94 & 6,57 & 17,56 \\
\hline 97 & KUTPO & 2,18 & 2,62 & 2,15 & 7,79 & 2,45 & 6,08 & 2,72 & 9,51 \\
\hline 98 & LUKSK & 1,24 & 1,45 & 1,20 & 1,77 & 1,29 & 2,57 & 1,66 & 2,82 \\
\hline 99 & MAKTK & 1,72 & 1,72 & 2,46 & 2,77 & 3,12 & 3,10 & 3,40 & 5,28 \\
\hline 100 & MRSHL & 0,85 & 1,10 & 0,79 & 1,32 & 0,97 & 4,94 & 1,92 & 5,12 \\
\hline 101 & MNDRS & 0,32 & 0,21 & 0,28 & 0,53 & 0,46 & 0,77 & 0,40 & 0,58 \\
\hline 102 & OLMK & 0,81 & 1,83 & 0,60 & 1,79 & 0,75 & 3,07 & 0,56 & 3,63 \\
\hline 103 & TIRE & 0,90 & 1,82 & 0,73 & 1,84 & 0,84 & 1,79 & 1,37 & 1,88 \\
\hline 104 & NIBAS & 13,70 & 7,73 & 12,15 & 11,71 & 15,04 & 26,53 & 9,38 & 64,49 \\
\hline 105 & NUHCM & 1,41 & 2,22 & 1,21 & 2,77 & 1,43 & 3,25 & 2,23 & 6,93 \\
\hline 106 & OTKAR & 1,15 & 1,21 & 1,68 & 1,57 & 1,80 & 1,32 & 1,82 & 2,20 \\
\hline 107 & OYLUM & 0,24 & 2,68 & 0,82 & 2,43 & 0,97 & 3,17 & 1,44 & 3,00 \\
\hline 108 & OZBAL & $-1,68$ & $-0,07$ & $-1,72$ & 0,64 & $-1,78$ & 1,13 & $-0,75$ & 0,78 \\
\hline 109 & PARSN & 0,50 & 0,58 & 0,74 & 0,74 & 0,56 & 0,75 & 0,75 & 0,92 \\
\hline 110 & PENGD & $-0,20$ & 0,11 & $-0,17$ & $-0,02$ & 0,09 & 3,16 & 0,01 & 3,96 \\
\hline 111 & PETKM & 1,03 & 0,94 & 1,18 & 1,05 & 1,07 & 1,00 & 1,00 & 1,29 \\
\hline 112 & PETUN & 1,35 & 1,63 & 1,52 & 2,18 & 1,69 & 2,43 & 1,86 & 2,46 \\
\hline 113 & PINSU & $-0,43$ & $-0,39$ & $-0,37$ & $-0,17$ & $-0,21$ & 0,15 & $-0,25$ & $-0,12$ \\
\hline 114 & PNSUT & 0,92 & 1,14 & 0,88 & 1,18 & 1,01 & 1,24 & 1,18 & 1,28 \\
\hline 115 & PRZMA & 4,15 & 12,33 & 4,49 & 9,65 & 8,29 & 3,29 & 8,93 & 5,56 \\
\hline 116 & ROYAL & $-0,04$ & 0,23 & 0,23 & 0,18 & 0,25 & 0,47 & 0,22 & 0,51 \\
\hline 117 & RTLAB & 4,35 & 6,13 & 3,66 & 18,64 & 6,95 & 18,88 & 5,12 & 9,54 \\
\hline 118 & SANFM & 0,31 & 0,80 & 0,28 & 0,82 & 0,40 & 1,19 & 0,81 & 1,53 \\
\hline 119 & SAMAT & 0,33 & 0,07 & 0,51 & 1,32 & 0,80 & 1,51 & 0,23 & 3,61 \\
\hline 120 & SARKY & 1,39 & 1,54 & 1,23 & 1,56 & 1,15 & 1,89 & 1,32 & 1,96 \\
\hline 121 & SAYAS & 0,34 & 1,76 & 0,61 & 4,16 & 0,95 & 2,97 & 1,50 & 2,21 \\
\hline 122 & SASA & 0,89 & 0,60 & 0,74 & 0,77 & 0,42 & 0,78 & 0,41 & 1,24 \\
\hline 123 & SEKUR & 0,79 & 0,59 & 0,49 & 0,68 & 0,53 & 0,94 & 0,71 & 0,98 \\
\hline 124 & SELGD & 3,10 & 4,39 & 2,88 & 5,71 & 3,25 & 18,12 & 5,27 & 32,95 \\
\hline 125 & SILVR & 0,57 & 0,79 & 0,81 & 1,09 & 0,85 & 1,17 & 1,02 & 1,74 \\
\hline 126 & SKTAS & $-0,08$ & $-0,59$ & $-0,44$ & $-0,55$ & $-0,53$ & $-0,14$ & $-0,37$ & $-0,04$ \\
\hline 127 & SNPAM & 2,81 & 4,42 & 3,23 & 6,90 & 3,64 & 10,73 & 4,56 & 13,80 \\
\hline 128 & TATGD & 2,25 & 2,68 & 1,99 & 2,87 & 1,95 & 2,45 & 2,35 & 2,42 \\
\hline 129 & TMPOL & 1,07 & 1,09 & 0,89 & 1,34 & 0,95 & 1,39 & 1,23 & 1,57 \\
\hline 130 & TOASO & 1,19 & 1,29 & 1,31 & 1,43 & 1,46 & 1,34 & 1,67 & 1,58 \\
\hline 131 & TUCLK & 0,64 & 0,73 & 0,58 & 0,79 & 0,64 & 1,07 & 0,73 & 1,02 \\
\hline 132 & TUKAS & 1,72 & 3,53 & 2,15 & 3,39 & 1,78 & 2,63 & 4,15 & 2,72 \\
\hline 133 & TMSN & 1,36 & 0,84 & 1,19 & 1,18 & 1,23 & 1,44 & 1,23 & 2,28 \\
\hline 134 & TUPRS & 1,07 & 0,62 & 1,11 & 0,66 & 1,14 & 0,84 & 0,98 & 0,88 \\
\hline 135 & PRKAB & 1,14 & 1,66 & 1,12 & 2,21 & 1,32 & 2,88 & 1,67 & 3,38 \\
\hline 136 & TTRAK & 1,12 & 1,46 & 1,07 & 2,15 & 1,47 & 2,32 & 1,82 & 3,17 \\
\hline 137 & TBORG & 1,42 & 2,38 & 1,42 & 2,47 & 2,10 & 3,22 & 2,66 & 3,13 \\
\hline 138 & ULUSE & 17,72 & 10,26 & 13,40 & 12,17 & 13,08 & 16,44 & 11,62 & 10,96 \\
\hline 139 & ULUUN & 1,29 & 1,19 & 1,23 & 1,34 & 1,23 & 1,15 & 1,13 & 1,21 \\
\hline 140 & USAK & 0,33 & 0,48 & 0,37 & 0,86 & 0,37 & 0,99 & 0,52 & 1,08 \\
\hline 141 & ULKER & 1,59 & 1,18 & 1,25 & 1,62 & 1,23 & 1,67 & 1,27 & 1,56 \\
\hline 142 & VESBE & 1,19 & 1,20 & 1,21 & 1,69 & 1,13 & 1,70 & 1,33 & 1,89 \\
\hline 143 & VESTL & 0,12 & 0,14 & 0,20 & 0,28 & 0,15 & 0,28 & 0,20 & 0,55 \\
\hline 144 & VKING & $-0,72$ & $-0,57$ & $-0,81$ & $-0,49$ & $-0,86$ & $\begin{array}{l}-0,58 \\
\end{array}$ & $-0,70$ & $-0,31$ \\
\hline 145 & YATAS & 1,53 & 1,68 & 1,65 & 1,85 & 1,83 & 2,06 & 1,94 & 2,52 \\
\hline 146 & YUNSA & 0,70 & 1,27 & 0,77 & 1,36 & 0,62 & 1,72 & 1,31 & 1,58 \\
\hline
\end{tabular}

Source: Authors' Compilation

The companies' determination within the research scope according to the Altman $\mathrm{Z}$ values in Table 4 for the years 2019-2020 according to the threshold values (Table 3) is given in Table 5. Again, RoFF (Risk of Financial Failure) was used for companies with a risk of financial failure, NRoFF (No Risk of Financial Failure) for companies with no risk of financial failure, and UN (Uncertain) for companies with an uncertain risk of financial failure.

PAGE 93| Journal of Corporate Governance, Insurance, and Risk Management | 2021, VOL. 8, Series. 2 
Table 5: Financial Status According to Altman Z Values of Manufacturing Companies for 2019-2020

\begin{tabular}{|c|c|c|c|c|c|c|}
\hline No & & & & & $1 \operatorname{ltman} \mathrm{Z}-1$ & cial Status \\
\hline No. & Code & $2019 / 3$ & $2020 / 3$ & $2019 / 6$ & $2020 / 6$ & $2019 / 9$ \\
\hline 1 & ACSEL & NRoFF & NRoFF & NRoFF & NRoFF & NRoFF \\
\hline 2 & ADEL & RoFF & RoFF & RoFF & RoFF & RoFF \\
\hline 3 & AFYON & RoFF & RoFF & RoFF & NRoFF & RoFF \\
\hline 4 & AKCNS & RoFF & RoFF & RoFF & RoFF & RoFF \\
\hline 5 & ATEKS & RoFF & RoFF & RoFF & RoFF & RoFF \\
\hline 6 & AKSA & RoFF & RoFF & RoFF & RoFF & RoFF \\
\hline 7 & ALCAR & NRoFF & UC & UC & NRoFF & NRoFF \\
\hline 8 & ALKA & RoFF & NRoFF & UC & NRoFF & UC \\
\hline 9 & ALKİM & $\mathrm{UC}$ & UC & $\mathrm{UC}$ & NRoFF & UC \\
\hline 10 & AEFES & RoFF & RoFF & RoFF & RoFF & RoFF \\
\hline 11 & ARCLK & RoFF & RoFF & RoFF & RoFF & RoFF \\
\hline 12 & ARSAN & RoFF & RoFF & RoFF & RoFF & RoFF \\
\hline 13 & ASUZU & RoFF & RoFF & RoFF & RoFF & RoFF \\
\hline 14 & AVOD & RoFF & RoFF & RoFF & RoFF & RoFF \\
\hline 15 & AYGAZ & RoFF & RoFF & RoFF & RoFF & RoFF \\
\hline 16 & BAGFS & RoFF & RoFF & RoFF & RoFF & RoFF \\
\hline 17 & BAKAB & RoFF & RoFF & RoFF & RoFF & RoFF \\
\hline 18 & BANVT & RoFF & RoFF & UC & UC & UC \\
\hline 19 & BRKSN & RoFF & RoFF & RoFF & RoFF & RoFF \\
\hline 20 & BLCYT & RoFF & NRoFF & RoFF & NRoFF & RoFF \\
\hline 21 & BNTAS & NRoFF & NRoFF & UC & NRoFF & NRoFF \\
\hline 22 & BSOKE & RoFF & RoFF & RoFF & RoFF & RoFF \\
\hline 23 & BTCIM & RoFF & RoFF & RoFF & RoFF & RoFF \\
\hline 24 & BRKO & RoFF & RoFF & RoFF & RoFF & RoFF \\
\hline 25 & BRMEN & RoFF & RoFF & RoFF & RoFF & RoFF \\
\hline 26 & BRSAN & RoFF & RoFF & RoFF & RoFF & RoFF \\
\hline 27 & BOSSA & RoFF & RoFF & RoFF & RoFF & RoFF \\
\hline 28 & BFREN & NRoFF & NRoFF & NRoFF & NRoFF & NRoFF \\
\hline 29 & BRISA & RoFF & RoFF & RoFF & RoFF & RoFF \\
\hline 30 & BURCE & RoFF & RoFF & RoFF & RoFF & RoFF \\
\hline 31 & BURVA & RoFF & NRoFF & RoFF & NRoFF & UC \\
\hline 32 & BUCIM & RoFF & UC & RoFF & UC & RoFF \\
\hline 33 & CCOLA & RoFF & RoFF & RoFF & RoFF & RoFF \\
\hline 34 & CELHA & RoFF & RoFF & RoFF & RoFF & RoFF \\
\hline 35 & CEMAS & RoFF & UC & RoFF & NRoFF & RoFF \\
\hline 36 & CEMTS & NRoFF & NRoFF & NRoFF & NRoFF & NRoFF \\
\hline 37 & CMBTN & RoFF & RoFF & RoFF & RoFF & RoFF \\
\hline 38 & CMENT & UC & RoFF & UC & UC & UC \\
\hline 39 & CIMSA & RoFF & RoFF & RoFF & RoFF & RoFF \\
\hline 40 & CUSAN & RoFF & RoFF & RoFF & UC & RoFF \\
\hline 41 & DAGI & RoFF & RoFF & RoFF & RoFF & RoFF \\
\hline 42 & DARDL & RoFF & RoFF & RoFF & RoFF & RoFF \\
\hline 43 & DMSAS & RoFF & RoFF & RoFF & RoFF & RoFF \\
\hline 44 & DERIM & RoFF & RoFF & RoFF & RoFF & RoFF \\
\hline 45 & DESA & RoFF & RoFF & RoFF & RoFF & RoFF \\
\hline 46 & DEVA & RoFF & UC & RoFF & UC & RoFF \\
\hline 47 & DIRIT & RoFF & RoFF & RoFF & RoFF & RoFF \\
\hline 48 & DITAS & RoFF & RoFF & RoFF & RoFF & RoFF \\
\hline 49 & DOBUR & RoFF & RoFF & $\mathrm{UC}$ & NRoFF & RoFF \\
\hline 50 & DGKLB & RoFF & RoFF & RoFF & RoFF & RoFF \\
\hline 51 & DOGUB & RoFF & UC & NRoFF & NRoFF & NRoFF \\
\hline 52 & DOKTA & RoFF & RoFF & RoFF & RoFF & RoFF \\
\hline 53 & DURDO & RoFF & RoFF & RoFF & RoFF & RoFF \\
\hline 54 & DYOBY & RoFF & RoFF & RoFF & RoFF & RoFF \\
\hline 55 & EGEEN & NRoFF & NRoFF & NRoFF & NRoFF & NRoFF \\
\hline 56 & EGGUB & RoFF & RoFF & RoFF & RoFF & RoFF \\
\hline 57 & EGPRO & RoFF & RoFF & RoFF & RoFF & RoFF \\
\hline 58 & EGESER & UC & RoFF & UC & UC & $\mathrm{UC}$ \\
\hline 59 & EPLAS & RoFF & NRoFF & RoFF & NRoFF & RoFF \\
\hline 60 & EKİZ & RoFF & RoFF & RoFF & RoFF & RoFF \\
\hline 61 & EMKEL & RoFF & RoFF & RoFF & RoFF & RoFF \\
\hline 62 & EMNIS & RoFF & RoFF & RoFF & RoFF & RoFF \\
\hline 63 & ERBOS & UC & $\mathrm{UC}$ & UC & NRoFF & UC \\
\hline 64 & EREGL & RoFF & RoFF & RoFF & RoFF & RoFF \\
\hline 65 & ERSU & RoFF & NRoFF & RoFF & NRoFF & RoFF \\
\hline 66 & FMZiP & NRoFF & NRoFF & NRoFF & NRoFF & NRoFF \\
\hline 67 & FROTO & $\mathrm{UC}$ & $\mathrm{UC}$ & UC & $\mathrm{UC}$ & $\mathrm{UC}$ \\
\hline 68 & FORMT & UC & RoFF & UC & RoFF & RoFF \\
\hline 69 & FRİGO & RoFF & RoFF & RoFF & UC & RoFF \\
\hline 70 & GEDZA & UC & UC & UC & NRoFF & UC \\
\hline 71 & GENTS & UC & $\mathrm{UC}$ & UC & NRoFF & $\mathrm{UC}$ \\
\hline 72 & GEREL & RoFF & RoFF & RoFF & $\mathrm{UC}$ & RoFF \\
\hline 73 & GOODY & RoFF & RoFF & RoFF & $\mathrm{UC}$ & RoFF \\
\hline 74 & GOLTS & RoFF & RoFF & RoFF & RoFF & RoFF \\
\hline 75 & GUBRF & RoFF & RoFF & RoFF & UC & RoFF \\
\hline 76 & HATEK & RoFF & RoFF & RoFF & RoFF & RoFF \\
\hline 77 & HEKTS & RoFF & RoFF & RoFF & RoFF & RoFF \\
\hline 78 & HURGZ & RoFF & RoFF & RoFF & RoFF & RoFF \\
\hline 79 & IHEVA & $\mathrm{UC}$ & NRoFF & $\mathrm{UC}$ & NRoFF & NRoFF \\
\hline 80 & IHGZT & RoFF & NRoFF & RoFF & $\mathrm{UC}$ & $\mathrm{UC}$ \\
\hline 81 & ISDMR & RoFF & UC & UC & $\mathrm{UC}$ & $\mathrm{UC}$ \\
\hline 82 & IZDMC & RoFF & RoFF & RoFF & RoFF & RoFF \\
\hline 83 & JANTS & RoFF & RoFF & RoFF & RoFF & RoFF \\
\hline 84 & KAPLM & RoFF & RoFF & RoFF & RoFF & RoFF \\
\hline 85 & KARDMD & RoFF & RoFF & RoFF & RoFF & RoFF \\
\hline 86 & KARSN & RoFF & RoFF & RoFF & RoFF & RoFF \\
\hline 87 & KRTEK & RoFF & RoFF & RoFF & RoFF & RoFF \\
\hline 88 & KARTN & RoFF & RoFF & RoFF & RoFF & RoFF \\
\hline 89 & KATMR & RoFF & RoFF & RoFF & RoFF & RoFF \\
\hline 90 & KENT & RoFF & $\mathrm{UC}$ & RoFF & $\mathrm{UC}$ & RoFF \\
\hline
\end{tabular}

PAGE 94| Journal of Corporate Governance, Insurance, and Risk Management | 2021, VOL. 8, Series. 2 


\begin{tabular}{|c|c|c|c|c|c|c|c|c|c|}
\hline 91 & KERVT & RoFF & $\mathrm{UC}$ & RoFF & $\mathrm{UC}$ & RoFF & $\mathrm{UC}$ & RoFF & $\mathrm{UC}$ \\
\hline 92 & KLMSN & RoFF & RoFF & RoFF & RoFF & RoFF & RoFF & RoFF & RoFF \\
\hline 93 & KONFRT & RoFF & RoFF & $\mathrm{UC}$ & $\mathrm{UC}$ & NRoFF & $\mathrm{UC}$ & RoFF & $\mathrm{UC}$ \\
\hline 94 & KONYA & NRoFF & NRoFF & NRoFF & NRoFF & NRoFF & NRoFF & NRoFF & NRoFF \\
\hline 95 & KORDS & RoFF & RoFF & RoFF & RoFF & RoFF & RoFF & RoFF & RoFF \\
\hline 96 & KRSTL & NRoFF & NRoFF & NRoFF & NRoFF & NRoFF & NRoFF & NRoFF & NRoFF \\
\hline 97 & KUTPO & $\mathrm{UC}$ & $\mathrm{UC}$ & UC & NRoFF & $\mathrm{UC}$ & NRoFF & UC & NRoFF \\
\hline 98 & LUKSK & RoFF & RoFF & RoFF & RoFF & RoFF & $\mathrm{UC}$ & RoFF & $\mathrm{UC}$ \\
\hline 99 & MAKTK & RoFF & RoFF & $\mathrm{UC}$ & UC & NRoFF & NRoFF & NRoFF & NRoFF \\
\hline 100 & MRSHL & RoFF & RoFF & RoFF & RoFF & RoFF & NRoFF & UC & NRoFF \\
\hline 101 & MNDRS & RoFF & RoFF & RoFF & RoFF & RoFF & RoFF & RoFF & RoFF \\
\hline 102 & OLMK & RoFF & $\mathrm{UC}$ & RoFF & RoFF & RoFF & NRoFF & RoFF & NRoFF \\
\hline 103 & TIRE & RoFF & $\mathrm{UC}$ & RoFF & $\mathrm{UC}$ & RoFF & RoFF & RoFF & $\mathrm{UC}$ \\
\hline 104 & NIBAS & NRoFF & NRoFF & NRoFF & NRoFF & NRoFF & NRoFF & NRoFF & NRoFF \\
\hline 105 & NUHCM & RoFF & $\mathrm{UC}$ & RoFF & $\mathrm{UC}$ & RoFF & NRoFF & $\mathrm{UC}$ & NRoFF \\
\hline 106 & OTKAR & RoFF & RoFF & RoFF & RoFF & RoFF & RoFF & $\mathrm{UC}$ & $\mathrm{UC}$ \\
\hline 107 & OYLUM & RoFF & $\mathrm{UC}$ & RoFF & $\mathrm{UC}$ & RoFF & NRoFF & RoFF & NRoFF \\
\hline 108 & OZBAL & RoFF & RoFF & RoFF & RoFF & RoFF & RoFF & RoFF & RoFF \\
\hline 109 & PARSN & RoFF & RoFF & RoFF & RoFF & RoFF & RoFF & RoFF & RoFF \\
\hline 110 & PENGD & RoFF & RoFF & RoFF & RoFF & RoFF & NRoFF & RoFF & NRoFF \\
\hline 111 & PETKM & RoFF & RoFF & RoFF & RoFF & RoFF & RoFF & RoFF & RoFF \\
\hline 112 & PETUN & RoFF & RoFF & RoFF & $\mathrm{UC}$ & RoFF & $\mathrm{UC}$ & $\mathrm{UC}$ & $\mathrm{UC}$ \\
\hline 113 & PINSU & RoFF & RoFF & RoFF & RoFF & RoFF & RoFF & RoFF & RoFF \\
\hline 114 & PNSUT & RoFF & RoFF & RoFF & RoFF & RoFF & RoFF & RoFF & RoFF \\
\hline 115 & PRZMA & NRoFF & NRoFF & NRoFF & NRoFF & NRoFF & NRoFF & NRoFF & NRoFF \\
\hline 116 & ROYAL & RoFF & RoFF & RoFF & RoFF & RoFF & RoFF & RoFF & RoFF \\
\hline 117 & RTLAB & NRoFF & NRoFF & NRoFF & NRoFF & NRoFF & NRoFF & NRoFF & NRoFF \\
\hline 118 & SANFM & RoFF & RoFF & RoFF & RoFF & RoFF & RoFF & RoFF & RoFF \\
\hline 119 & SAMAT & RoFF & RoFF & RoFF & RoFF & RoFF & RoFF & RoFF & NRoFF \\
\hline 120 & SARKY & RoFF & RoFF & RoFF & RoFF & RoFF & UC & RoFF & $\mathrm{UC}$ \\
\hline 121 & SAYAS & RoFF & RoFF & RoFF & NRoFF & RoFF & $\mathrm{UC}$ & RoFF & UC \\
\hline 122 & SASA & RoFF & RoFF & RoFF & RoFF & RoFF & RoFF & RoFF & RoFF \\
\hline 123 & SEKUR & RoFF & RoFF & RoFF & RoFF & RoFF & RoFF & RoFF & RoFF \\
\hline 124 & SELGD & NRoFF & NRoFF & $\mathrm{UC}$ & NRoFF & $\mathrm{NRoFF}$ & $\mathrm{NRoFF}$ & NRoFF & NRoFF \\
\hline 125 & SILVR & RoFF & RoFF & RoFF & RoFF & RoFF & RoFF & RoFF & RoFF \\
\hline 126 & SKTAS & RoFF & RoFF & RoFF & RoFF & RoFF & RoFF & RoFF & RoFF \\
\hline 127 & SNPAM & UC & NRoFF & NRoFF & NRoFF & NRoFF & NRoFF & NRoFF & NRoFF \\
\hline 128 & TATGD & UC & UC & $\mathrm{UC}$ & $\mathrm{UC}$ & UC & $\mathrm{UC}$ & $\mathrm{UC}$ & UC \\
\hline 129 & TMPOL & RoFF & RoFF & RoFF & RoFF & RoFF & RoFF & RoFF & RoFF \\
\hline 130 & TOASO & RoFF & RoFF & RoFF & RoFF & RoFF & RoFF & RoFF & RoFF \\
\hline 131 & TUCLK & RoFF & RoFF & RoFF & RoFF & RoFF & RoFF & RoFF & RoFF \\
\hline 132 & TUKAS & RoFF & NRoFF & UC & NRoFF & RoFF & UC & NRoFF & UC \\
\hline 133 & TMSN & RoFF & RoFF & RoFF & RoFF & RoFF & RoFF & RoFF & UC \\
\hline 134 & TUPRS & RoFF & RoFF & RoFF & RoFF & RoFF & RoFF & RoFF & RoFF \\
\hline 135 & PRKAB & RoFF & RoFF & RoFF & $\mathrm{UC}$ & RoFF & $\mathrm{UC}$ & RoFF & NRoFF \\
\hline 136 & TTRAK & RoFF & RoFF & RoFF & UC & RoFF & $\mathrm{UC}$ & $\mathrm{UC}$ & NRoFF \\
\hline 137 & TBORG & RoFF & $\mathrm{UC}$ & RoFF & $\mathrm{UC}$ & $\mathrm{UC}$ & NRoFF & UC & NRoFF \\
\hline 138 & ULUSE & NRoFF & NRoFF & NRoFF & NRoFF & NRoFF & NRoFF & NRoFF & NRoFF \\
\hline 139 & ULUUN & RoFF & RoFF & RoFF & RoFF & RoFF & RoFF & RoFF & RoFF \\
\hline 140 & USAK & RoFF & RoFF & RoFF & RoFF & RoFF & RoFF & RoFF & RoFF \\
\hline 141 & ULKER & RoFF & RoFF & RoFF & RoFF & RoFF & RoFF & RoFF & RoFF \\
\hline 142 & VESBE & RoFF & RoFF & RoFF & RoFF & RoFF & RoFF & RoFF & UC \\
\hline 143 & VESTL & RoFF & RoFF & RoFF & RoFF & RoFF & RoFF & RoFF & RoFF \\
\hline 144 & VKING & RoFF & RoFF & RoFF & RoFF & RoFF & RoFF & RoFF & RoFF \\
\hline 145 & YATAS & RoFF & RoFF & RoFF & UC & UC & UC & UC & UC \\
\hline 146 & YUNSA & RoFF & RoFF & RoFF & RoFF & RoFF & RoFF & RoFF & RoFF \\
\hline \multirow{3}{*}{ TOTAL } & NO RISK OF FAILURE & 14 & 22 & 13 & 32 & 19 & 41 & 22 & 51 \\
\hline & RISK OF FINANCIAL FAILURE & 120 & 104 & 112 & 88 & 111 & 75 & 96 & 64 \\
\hline & UNCERTAIN & 12 & 20 & 21 & 26 & 16 & 30 & 28 & 31 \\
\hline
\end{tabular}

Source: Authors' Compilation

When Table 5 is examined, it is seen that in the period of 2019/3, when the number of financially unsuccessful firms was the highest, 120 of 146 firms failed Altman Z scores. According to the Altman Z-score, it is seen that the period with the highest success belongs to 2020/12 where 51 out of 146 companies are successful. The companies that have been successful in each quarter with the Altman $\mathrm{Z}$ Score calculated for the period 2019-2020 are: ACSEL, BFREN, CEMTS, EGEEN, FMZIP, KONYA, KRSTL, NIBAS, PRZMA, RTLAB, ULUSE. Companies that failed in terms of Altman Z Score in all periods are: ADEL, AKSA, AEFES, ARCLK, BAGFS, BSOKE, BTCIM, BRKO, BRMEN, BRSAN, BOSSA, BRISA, BURCE, CCOLA, CELHA, CIMSA, DAGI, DARDNL, DERIM, DESA, DIRIT, DITAS, DGKLB, DOKTA, DURDO, DYOBY, EKIZ, EMKEL, GOLTS, HATEK, HEKTS, IZDMC, KARDMD, KARSN, KRTEK, KATMR, KLMSN, KORDS, MNDRS, PETKM, PINSU, PNSUT, ROYAL, SANFM, SASA, SEKUR, SILVR, SKTAS, TMPOL, TOASO, TUCLK, TUPRS, ULUUN, USAK, ULKER, VESTL, VKING, YUNSA.

PAGE 95| Journal of Corporate Governance, Insurance, and Risk Management | 2021, VOL. 8, Series. 2 
According to the formula in Table 3, the Springate model's values of the BIST manufacturing companies within the scope of the research for the years 2019-2020 are given in Table 6.

Table 6: Springate Values of BIST Manufacturing Companies for 2019-2020

\begin{tabular}{|c|c|c|c|c|c|c|c|c|c|}
\hline \multirow{2}{*}{ No. } & & & & & & & & & \\
\hline & Code & $2019 / 3$ & $2020 / 3$ & $2019 / 6$ & $2020 / 6$ & $2019 / 9$ & $2020 / 9$ & $2019 / 12$ & $2020 / 12$ \\
\hline 1 & ACSEL & 0,86 & 0,85 & 1,67 & 1,16 & $\begin{array}{l}0,91 \\
\end{array}$ & 1,09 & 0,96 & 1,38 \\
\hline 2 & ADEL & 0,83 & 0,68 & 0,61 & 0,66 & 0,62 & 0,65 & 0,50 & 0,42 \\
\hline 3 & AFYON & $-0,31$ & $-0,36$ & $-0,30$ & 0,05 & $-0,26$ & 0,10 & $-0,35$ & 0,06 \\
\hline 4 & AKCNS & 0,16 & 0,17 & 0,25 & 0,37 & 0,29 & 0,48 & 0,35 & 0,43 \\
\hline 5 & ATEKS & 0,28 & 0,22 & 0,29 & 0,14 & 0,25 & 0,24 & 0,30 & 0,55 \\
\hline 6 & AKSA & 0,38 & 0,34 & 0,34 & 0,19 & 0,34 & 0,51 & 0,28 & 0,69 \\
\hline 7 & ALCAR & 1,08 & 1,07 & 1,08 & 1,10 & 1,21 & 1,28 & 1,14 & 1,22 \\
\hline 8 & ALKA & 0,74 & 1,15 & 1,14 & 1,68 & 1,08 & 1,43 & 1,23 & 1,25 \\
\hline 9 & ALKİM & 1,05 & 1,27 & 1,22 & 1,30 & 1,17 & 1,26 & 1,23 & 1,16 \\
\hline 10 & AEFES & 0,27 & 0,33 & 0,48 & 0,47 & 0,53 & 0,56 & 0,42 & 0,43 \\
\hline 11 & ARCLK & 0,76 & 0,77 & 0,80 & 0,77 & 0,82 & 0,82 & 0,80 & 0,90 \\
\hline 12 & ARSAN & 0,21 & 0,44 & 0,27 & 0,24 & 0,33 & 0,61 & 0,20 & 0,77 \\
\hline 13 & ASUZU & 0,30 & 0,28 & 0,30 & 0,22 & 0,23 & 0,28 & 0,50 & 0,51 \\
\hline 14 & AVOD & 0,35 & 0,34 & 0,44 & 0,21 & 0,42 & 0,22 & 0,24 & 0,18 \\
\hline 15 & AYGAZ & 0,41 & 0,30 & 0,52 & 0,49 & 0,53 & 0,62 & 0,56 & 0,60 \\
\hline 16 & BAGFS & 0,25 & 0,21 & 0,20 & 0,16 & 0,31 & 0,13 & 0,05 & 0,30 \\
\hline 17 & BAKAB & 0,48 & 0,53 & 0,50 & 0,58 & 0,48 & 0,55 & 0,45 & 0,64 \\
\hline 18 & BANVT & 0,74 & 0,65 & 1,22 & 0,72 & 0,86 & 0,63 & 0,70 & 0,59 \\
\hline 19 & BRKSN & 0,73 & 0,51 & 0,39 & 0,47 & $\begin{array}{l}0,69 \\
\end{array}$ & 0,94 & 0,42 & 1,06 \\
\hline 20 & BLCYT & 0,65 & 0,89 & 0,73 & 0,39 & 0,92 & 0,94 & 1,38 & 0,82 \\
\hline 21 & BNTAS & 0,79 & 0,94 & 0,85 & 0,95 & 0,73 & 1,09 & 1,01 & 0,74 \\
\hline 22 & BSOKE & $-0,03$ & $-0,12$ & $-0,21$ & $-0,17$ & $-0,04$ & $-0,21$ & $-0,32$ & $-0,14$ \\
\hline 23 & BTCIM & 0,02 & $\begin{array}{l}-0,04 \\
\end{array}$ & $-0,04$ & $\begin{array}{c}-0,08 \\
\end{array}$ & 0,03 & $-0,09$ & $-0,15$ & $-0,02$ \\
\hline 24 & BRKO & $-0,05$ & $\begin{array}{l}-0,10 \\
\end{array}$ & $\begin{array}{l}-0,01 \\
\end{array}$ & $\begin{array}{l}-0,24 \\
\end{array}$ & $-0,23$ & $\begin{array}{l}-0,01 \\
\end{array}$ & $-0,16$ & 0,03 \\
\hline 25 & BRMEN & $-0,62$ & $\begin{array}{l}-1,39 \\
\end{array}$ & $-0,65$ & 0,09 & $-0,69$ & $-1,13$ & $-0,66$ & $-0,85$ \\
\hline 26 & BRSAN & 0,23 & 0,15 & 0,27 & 0,07 & 0,18 & 0,11 & 0,22 & 0,18 \\
\hline 27 & BOSSA & 0,39 & 0,37 & 0,44 & 0,19 & 0,47 & 0,44 & 0,46 & 0,71 \\
\hline 28 & BFREN & 1,61 & 1,26 & 1,44 & 0,62 & 0,85 & 1,29 & 1,06 & 1,04 \\
\hline 29 & BRİSA & 0,32 & 0,25 & 0,34 & 0,18 & 0,39 & 0,38 & 0,27 & 0,66 \\
\hline 30 & BURCE & 0,14 & 0,06 & 0,20 & 0,07 & 0,24 & 0,31 & 0,18 & 0,24 \\
\hline 31 & BURVA & 0,68 & 0,50 & 0,73 & 0,43 & 0,88 & 0,82 & 0,85 & 0,80 \\
\hline 32 & BUCIM & 0,79 & 0,78 & 0,86 & 0,76 & 0,70 & 0,98 & 0,76 & 1,12 \\
\hline 33 & CCOLA & 0,46 & 0,52 & 0,68 & 0,66 & 0,75 & 0,87 & 0,50 & 0,58 \\
\hline 34 & CELHA & 0,30 & 0,29 & 0,44 & 0,11 & 0,33 & 0,30 & 0,26 & 0,42 \\
\hline 35 & CEMAS & 0,26 & 0,60 & 0,33 & 1,00 & 0,22 & 0,83 & $-0,10$ & $-0,05$ \\
\hline 36 & CEMTS & 1,00 & 1,05 & 1,25 & 1,20 & 0,86 & 1,22 & 1,14 & 1,59 \\
\hline 37 & CMBTN & 0,49 & 0,20 & 0,41 & 0,18 & 0,45 & 0,24 & 0,44 & 0,26 \\
\hline 38 & $\begin{array}{l}\text { CMENT } \\
\end{array}$ & 0,15 & 0,13 & 0,25 & 0,13 & 0,24 & 0,31 & 0,27 & 0,42 \\
\hline 39 & CIMSA & 0,04 & 0,07 & 0,03 & 0,15 & 0,12 & 0,13 & 0,08 & 0,08 \\
\hline 40 & CUSAN & 0,53 & 0,74 & 0,68 & 0,68 & 0,62 & 0,70 & 0,68 & 0,75 \\
\hline 41 & DAGI & 0,54 & 0,59 & 0,67 & 0,45 & 0,68 & 0,61 & 0,76 & 0,50 \\
\hline 42 & DARDL & $-0,02$ & 1,31 & 0,34 & 0,84 & 0,51 & 0,40 & 0,83 & 0,60 \\
\hline 43 & DMSAS & 0,66 & 0,56 & 0,55 & 0,46 & 0,46 & 0,67 & 0,62 & 0,89 \\
\hline 44 & DERIMM & 0,54 & 0,54 & 0,47 & 0,43 & 0,49 & 0,49 & 0,64 & 0,46 \\
\hline 45 & DESA & 0,75 & 0,65 & 0,78 & 0,29 & 0,72 & 0,84 & 0,94 & 0,86 \\
\hline 46 & DEVA & 0,80 & 0,96 & 0,79 & 0,83 & 0,71 & 0,90 & 0,82 & 0,92 \\
\hline 47 & DIRIT & $-0,35$ & 0,13 & $-0,55$ & $-0,12$ & $-1,01$ & $-0,41$ & $-0,40$ & $-0,60$ \\
\hline 48 & DİTAS & 0,73 & 0,55 & 0,76 & 0,44 & 0,78 & 0,77 & 0,58 & 0,89 \\
\hline 49 & DOBUR & 1,64 & 1,13 & 1,35 & 1,73 & 1,15 & 1,20 & 1,51 & 1,61 \\
\hline 50 & DGKLB & 0,29 & 0,41 & 0,49 & 0,33 & 0,33 & 0,55 & 0,38 & 0,65 \\
\hline 51 & DOGUB & $-0,32$ & $-0,12$ & $-0,18$ & $-0,02$ & 0,02 & 0,07 & $-0,03$ & 0,14 \\
\hline 52 & DOKTA & 0,17 & 0,22 & 0,12 & 0,04 & 0,32 & 0,06 & 0,31 & 0,33 \\
\hline 53 & DURDO & 0,46 & 0,42 & 0,57 & 0,34 & 0,76 & 0,42 & 0,66 & 0,65 \\
\hline 54 & DYOBY & 0,38 & 0,31 & 0,44 & 0,45 & 0,40 & 0,66 & 0,48 & 0,70 \\
\hline 55 & EGEEN & 1,33 & 0,90 & 1,14 & 0,61 & 0,97 & 0,99 & 0,95 & 0,94 \\
\hline 56 & EGGUB & 0,57 & 0,39 & 0,57 & 0,32 & 0,55 & 0,38 & 0,13 & 0,40 \\
\hline 57 & EGPRO & 0,44 & 0,58 & 0,53 & 0,53 & 0,57 & 0,60 & 0,59 & 0,79 \\
\hline 58 & EGESER & 0,74 & 0,76 & 0,87 & 0,83 & 0,77 & 1,00 & 0,84 & 1,10 \\
\hline 59 & EPLAS & 0,71 & 1,18 & 0,69 & 0,98 & 0,77 & 1,24 & 0,73 & 1,40 \\
\hline 60 & EKİZ & $-0,32$ & $-0,17$ & $-0,27$ & $-0,30$ & $-0,17$ & $-0,29$ & $-0,17$ & $-0,04$ \\
\hline 61 & EMKEL & 0,01 & 0,03 & 0,04 & 0,05 & 0,00 & 0,03 & 0,03 & 0,14 \\
\hline 62 & EMNIS & $-0,41$ & 0,25 & 0,17 & 0,53 & 0,11 & 0,73 & 0,00 & 1,86 \\
\hline 63 & ERBOS & 1,17 & 0,90 & 1,19 & 0,92 & 0,95 & 1,10 & 0,72 & 1,31 \\
\hline 64 & EREGL & 0,56 & 0,52 & 0,69 & 0,53 & 0,58 & 0,56 & 0,49 & 0,72 \\
\hline 65 & ERSU & 0,23 & 0,38 & 0,29 & 0,35 & 0,66 & 0,38 & 0,41 & 0,39 \\
\hline 66 & FMZIP & 2,15 & 2,09 & 2,75 & 1,42 & 2,02 & 1,58 & 2,14 & 2,28 \\
\hline 67 & FROTO & 0,54 & 0,63 & 0,57 & 0,43 & 0,63 & 0,78 & 0,73 & 1,11 \\
\hline 68 & FORMT & 0,65 & 0,46 & 0,68 & 0,54 & 0,67 & 0,43 & 0,77 & 0,61 \\
\hline 69 & FRIGO & 0,74 & 0,75 & 0,85 & 0,92 & 0,56 & 0,81 & 0,65 & 0,73 \\
\hline 70 & GEDZA & 0,89 & 0,91 & 0,90 & 0,94 & 0,81 & 0,93 & 0,80 & 1,06 \\
\hline 71 & GENTS & 0,72 & 0,91 & 0,92 & 0,88 & 0,88 & 1,27 & 0,81 & 1,38 \\
\hline 72 & GEREL & 0,58 & 0,54 & 0,59 & 0,62 & 0,59 & 0,60 & 0,44 & 0,60 \\
\hline 73 & GOODY & 0,56 & 0,67 & 0,68 & 0,67 & 0,67 & 1,16 & 1,22 & 0,94 \\
\hline 74 & GOLTS & 0,49 & 0,38 & 0,36 & 0,41 & 0,30 & 0,35 & 0,21 & 0,26 \\
\hline 75 & GUBRF & 0,34 & 0,56 & 0,27 & 0,32 & 0,25 & 0,43 & 0,21 & 0,47 \\
\hline 76 & HATEK & 0,18 & 0,07 & 0,15 & 0,19 & 0,24 & 0,27 & 0,40 & 0,53 \\
\hline 77 & HEKTS & 1,01 & 0,94 & 0,80 & 0,46 & 0,59 & 0,53 & 0,73 & 0,42 \\
\hline 78 & HURGZ & 0,46 & 0,32 & 0,36 & 0,13 & 0,26 & 0,28 & 0,42 & 0,18 \\
\hline 79 & IHEVA & 0,72 & 0,70 & 0,69 & 0,77 & 0,89 & 0,91 & 0,90 & 1,17 \\
\hline 80 & IHGZT & 0,40 & 0,11 & 0,36 & 0,30 & 0,48 & 0,33 & 1,18 & 0,49 \\
\hline 81 & ISDMR & 0,52 & 0,61 & 0,87 & 0,62 & 0,68 & 0,65 & 0,59 & 0,98 \\
\hline 82 & IZDMC & $-0,29$ & $-0,29$ & $-0,26$ & $-0,34$ & $-0,17$ & $-0,28$ & $-0,23$ & $-0,12$ \\
\hline 83 & JANTS & 0,88 & 0,80 & 1,05 & 0,96 & 0,64 & 1,04 & 0,80 & 1,21 \\
\hline 84 & KAPLM & 0,18 & 0,23 & 0,07 & 0,04 & $\begin{array}{l}-0,01 \\
\end{array}$ & 0,28 & 0,10 & 0,27 \\
\hline 85 & KARDMD & 0,22 & 0,08 & 0,27 & 0,10 & 0,20 & 0,14 & 0,10 & 0,37 \\
\hline
\end{tabular}

PAGE 96| Journal of Corporate Governance, Insurance, and Risk Management | 2021, VOL. 8, Series. 2 


\begin{tabular}{|c|c|c|c|c|c|c|c|c|c|}
\hline 86 & KARSN & 0,06 & 0,16 & 0,17 & 0,24 & 0,14 & 0,41 & 0,23 & 0,61 \\
\hline 87 & KRTEK & 0,50 & 0,42 & 0,53 & 0,32 & 0,57 & 0,41 & 0,30 & 0,43 \\
\hline 88 & KARTN & 1,16 & 1,03 & 1,12 & 1,13 & 0,93 & 1,32 & 0,97 & 1,35 \\
\hline 89 & $\begin{array}{l}\text { KATMR } \\
\end{array}$ & 0,43 & 0,74 & 0,78 & 0,75 & 0,84 & 0,91 & 0,84 & 0,86 \\
\hline 90 & KENT & 0,65 & 0,70 & 0,72 & 0,68 & 0,57 & 0,67 & 0,63 & 0,53 \\
\hline 91 & KERVT & 0,73 & 0,79 & 0,83 & 0,76 & 0,83 & 0,78 & 0,75 & 1,02 \\
\hline 92 & KLMSN & 0,81 & 0,60 & 0,84 & 0,72 & 0,67 & 0,70 & 0,73 & 0,79 \\
\hline 93 & $\begin{array}{l}\text { KONFRT } \\
\end{array}$ & 0,79 & 0,53 & 1,00 & 0,63 & 0,61 & 0,45 & 0,32 & 0,46 \\
\hline 94 & KONYA & 0,56 & 0,40 & 0,56 & 0,59 & 0,77 & 0,90 & 0,71 & 0,70 \\
\hline 95 & KORDS & 0,46 & 0,31 & 0,46 & 0,07 & 0,37 & 0,21 & 0,33 & 0,36 \\
\hline 96 & KRSTL & 0,79 & 0,79 & 0,66 & 0,78 & 0,74 & 0,78 & 0,67 & 0,51 \\
\hline 97 & KUTPO & 0,99 & 0,89 & 0,95 & 0,90 & 0,90 & 0,93 & 1,12 & 1,33 \\
\hline 98 & LUKSK & 0,44 & 0,37 & 0,57 & 0,36 & 0,45 & 0,54 & 0,52 & 0,83 \\
\hline 99 & $\begin{array}{l}\text { MAKTK } \\
\end{array}$ & 0,86 & 0,73 & 0,89 & 0,49 & 0,94 & 0,68 & 0,64 & 0,71 \\
\hline 100 & MRSHL & 0,65 & 0,59 & 0,63 & 0,52 & 0,60 & 0,49 & 0,84 & 0,51 \\
\hline 101 & MNDRS & 0,15 & 0,04 & 0,12 & 0,19 & 0,28 & 0,36 & 0,13 & 0,39 \\
\hline 102 & OLMK & 0,49 & 0,57 & 0,38 & 0,58 & 0,47 & 0,56 & 0,32 & 0,77 \\
\hline 103 & TIRE & 0,41 & 0,43 & 0,35 & 0,47 & 0,41 & 0,41 & 0,37 & 0,54 \\
\hline 104 & NiBAS & 0,15 & 0,24 & 0,16 & 0,17 & 0,27 & 0,10 & 0,46 & $-0,15$ \\
\hline 105 & NUHCM & 0,38 & 0,75 & 0,37 & 0,80 & 0,42 & 0,80 & 0,56 & 0,70 \\
\hline 106 & OTKAR & 0,58 & 0,68 & 1,16 & 0,81 & 1,08 & 0,67 & 0,89 & 0,99 \\
\hline 107 & OYLUM & 0,33 & 0,62 & 0,36 & 0,68 & 0,33 & 0,66 & 0,44 & 0,62 \\
\hline 108 & OZBAL & $-0,64$ & 0,06 & $-0,76$ & 0,19 & $-0,72$ & 0,16 & $-0,27$ & $-0,01$ \\
\hline 109 & PARSN & 0,13 & 0,13 & 0,07 & 0,04 & 0,02 & 0,05 & 0,03 & 0,27 \\
\hline 110 & PENGD & 0,01 & 0,04 & 0,13 & $-0,07$ & 0,25 & 0,45 & 0,03 & 0,56 \\
\hline 111 & PETKM & 0,59 & 0,51 & 0,62 & 0,56 & 0,56 & 0,57 & 0,51 & 0,62 \\
\hline 112 & PETUN & 0,36 & 0,46 & 0,47 & 0,42 & 0,55 & 0,42 & 0,47 & 0,47 \\
\hline 113 & PINSU & 0,10 & 0,09 & 0,19 & 0,10 & 0,35 & 0,27 & 0,14 & $-0,03$ \\
\hline 114 & PNSUT & 0,38 & 0,47 & 0,35 & 0,37 & 0,34 & 0,34 & 0,43 & 0,37 \\
\hline 115 & PRZMA & 0,32 & 0,71 & 0,41 & 0,41 & 0,54 & 0,46 & 0,60 & 0,64 \\
\hline 116 & ROYAL & 0,20 & 0,44 & 0,46 & 0,27 & 0,28 & 0,47 & 0,25 & 0,58 \\
\hline 117 & RTLAB & 0,78 & 0,96 & 1,15 & 1,64 & 0,09 & 1,05 & 0,34 & 1,32 \\
\hline 118 & SANFM & 0,24 & 0,33 & 0,25 & 0,32 & 0,32 & 0,38 & 0,37 & 0,46 \\
\hline 119 & SAMAT & 0,19 & 0,18 & 0,29 & 0,50 & 0,44 & 0,41 & $-0,02$ & 0,60 \\
\hline 120 & SARKY & 0,70 & 0,73 & 0,62 & 0,69 & 0,58 & 0,84 & 0,58 & 0,82 \\
\hline 121 & SAYAS & 0,42 & 0,87 & 0,66 & 1,35 & 0,70 & 0,79 & 0,69 & 1,19 \\
\hline 122 & SASA & 0,13 & $-0,16$ & 0,03 & $-0,04$ & $-0,11$ & $-0,10$ & $-0,15$ & 0,15 \\
\hline 123 & SEKUR & 0,57 & 0,37 & 0,40 & 0,40 & 0,36 & 0,31 & 0,42 & 0,26 \\
\hline 124 & SELGD & 0,67 & 0,47 & 0,67 & 0,45 & 0,68 & 0,69 & 1,04 & 0,85 \\
\hline 125 & SILVR & 0,16 & 0,40 & 0,33 & 0,27 & 0,33 & 0,46 & 0,42 & 0,50 \\
\hline 126 & SKTAS & 0,22 & $-0,08$ & 0,07 & $-0,16$ & $-0,01$ & $-0,02$ & 0,06 & 0,05 \\
\hline 127 & SNPAM & 0,33 & 0,39 & 0,32 & 0,09 & 0,25 & 0,21 & 0,77 & 0,59 \\
\hline 128 & TATGD & 1,01 & 1,22 & 1,00 & 1,06 & 0,88 & 0,87 & 1,04 & 0,58 \\
\hline 129 & TMPOL & 0,60 & 0,38 & 0,47 & 0,44 & 0,46 & 0,52 & 0,46 & 0,62 \\
\hline 130 & TOASO & 0,44 & 0,49 & 0,47 & 0,42 & 0,48 & 0,52 & 0,55 & 0,61 \\
\hline 131 & TUCLK & 0,26 & 0,22 & 0,23 & 0,17 & 0,29 & 0,26 & 0,17 & 0,41 \\
\hline 132 & TUKAS & 0,40 & 0,57 & 0,41 & 0,49 & 0,38 & 0,56 & 0,50 & 0,50 \\
\hline 133 & TMSN & 0,34 & 0,23 & 0,30 & 0,38 & 0,24 & 0,46 & 0,24 & 0,70 \\
\hline 134 & TUPRS & 0,29 & 0,01 & 0,36 & 0,16 & 0,29 & 0,28 & 0,24 & 0,23 \\
\hline 135 & PRKAB & 0,65 & 0,69 & 0,65 & 0,76 & 0,75 & 0,71 & 0,78 & 0,77 \\
\hline 136 & TTRAK & 0,51 & 0,71 & 0,47 & 0,78 & 0,60 & 0,96 & 0,80 & 1,21 \\
\hline 137 & TBORG & 0,53 & 0,61 & 0,70 & 0,77 & 1,16 & 1,14 & 1,04 & 1,00 \\
\hline 138 & ULUSE & 1,61 & 0,87 & 1,17 & 0,94 & 1,31 & 0,98 & 1,20 & 0,92 \\
\hline 139 & ULUUN & 0,67 & 0,62 & 0,72 & 0,63 & 0,61 & 0,56 & 0,61 & 0,66 \\
\hline 140 & USAK & 0,16 & 0,30 & 0,22 & 0,38 & 0,20 & 0,41 & 0,32 & 0,37 \\
\hline 141 & ULKER & 0,91 & 0,46 & 0,54 & 0,71 & 0,58 & 0,68 & 0,47 & 0,98 \\
\hline 142 & VESBE & 0,36 & 0,40 & 0,45 & 0,53 & 0,41 & 0,60 & 0,43 & 0,73 \\
\hline 143 & VESTL & 0,09 & 0,16 & 0,15 & 0,18 & 0,13 & 0,25 & 0,17 & 0,38 \\
\hline 144 & VKING & $-0,06$ & $-0,05$ & $-0,20$ & $-0,07$ & $-0,23$ & $-0,20$ & $-0,17$ & $-0,20$ \\
\hline 145 & YATAS & 0,88 & 1,08 & 1,15 & 0,83 & 1,06 & 1,34 & 1,22 & 1,41 \\
\hline 146 & YUNSA & 0,51 & 0,75 & 0,53 & 0,46 & 0,41 & 0,68 & 0,61 & 0,57 \\
\hline
\end{tabular}

Source: Authors' Compilation

The determination of the companies within the scope of the research according to the Springate values in Table 6 for the years 2019-2020 according to the threshold values (Table 3) is given in Table 7. RoFF (Risk of Financial Failure) was used for companies with a risk of financial failure and NRoFF (No Risk of Financial Failure) for companies with no risk of financial failure.

Table 7: Financial Status According to Springate Values of Manufacturing Companies for 2019-2020

\begin{tabular}{|c|c|c|c|c|c|c|c|c|c|}
\hline \multirow[b]{2}{*}{ No. } & \multirow{2}{*}{ Code } & \multicolumn{8}{|c|}{ Springate-Financial Status } \\
\hline & & $2019 / 3$ & $2020 / 3$ & $2019 / 6$ & $2020 / 6$ & $2019 / 9$ & $2020 / 9$ & $2019 / 12$ & $2020 / 12$ \\
\hline 1 & ACSEL & NRoFF & RoFF & $\mathrm{NRoFF}$ & NRoFF & NRoFF & NRoFF & NRoFF & NRoFF \\
\hline 2 & ADEL & RoFF & RoFF & RoFF & RoFF & RoFF & RoFF & RoFF & RoFF \\
\hline 3 & AFYLN & RoFF & RoFF & RoFF & RoFF & RoFF & RoFF & RoFF & RoFF \\
\hline 4 & AKCNS & RoFF & RoFF & RoFF & RoFF & RoFF & RoFF & RoFF & RoFF \\
\hline 5 & ATEKS & RoFF & RoFF & RoFF & RoFF & RoFF & RoFF & RoFF & RoFF \\
\hline 6 & AKSA & RoFF & RoFF & RoFF & RoFF & RoFF & RoFF & RoFF & RoFF \\
\hline 7 & ALCAR & NRoFF & NRoFF & NRoFF & NRoFF & NRoFF & NRoFF & NRoFF & NRoFF \\
\hline 8 & ALKA & RoFF & NRoFF & NRoFF & NRoFF & NRoFF & NRoFF & NRoFF & $\mathrm{NRoFF}$ \\
\hline 9 & ALKIM & NRoFF & NRoFF & NRoFF & NRoFF & NRoFF & NRoFF & NRoFF & NRoFF \\
\hline 10 & AEFES & RoFF & RoFF & RoFF & RoFF & RoFF & RoFF & RoFF & RoFF \\
\hline 11 & ARCLK & RoFF & RoFF & RoFF & RoFF & RoFF & RoFF & RoFF & $\mathrm{NRoFF}$ \\
\hline 12 & ARSAN & RoFF & RoFF & RoFF & RoFF & RoFF & RoFF & RoFF & RoFF \\
\hline 13 & ASUZU & RoFF & RoFF & RoFF & RoFF & RoFF & RoFF & RoFF & RoFF \\
\hline 14 & AVOD & RoFF & RoFF & RoFF & RoFF & RoFF & RoFF & RoFF & RoFF \\
\hline 15 & AYGAZ & RoFF & RoFF & RoFF & RoFF & RoFF & RoFF & RoFF & RoFF \\
\hline
\end{tabular}

PAGE 97| Journal of Corporate Governance, Insurance, and Risk Management | 2021, VOL. 8, Series. 2 


\begin{tabular}{|c|c|c|c|c|}
\hline 16 & BAGFS & RoFF & RoFF & RoFF \\
\hline 17 & BAKAB & RoFF & RoFF & RoFF \\
\hline 18 & BANVT & RoFF & RoFF & NRoFF \\
\hline 19 & BRKSN & RoFF & RoFF & RoFF \\
\hline 20 & BLCYT & RoFF & NRoFF & RoFF \\
\hline 21 & BNTAS & RoFF & NRoFF & RoFF \\
\hline 22 & BSOKE & RoFF & RoFF & RoFF \\
\hline 23 & BTCIM & RoFF & RoFF & RoFF \\
\hline 24 & BRKO & RoFF & RoFF & RoFF \\
\hline 25 & BRMEN & RoFF & RoFF & RoFF \\
\hline 26 & BRSAN & RoFF & RoFF & RoFF \\
\hline 27 & BOSSA & RoFF & RoFF & RoFF \\
\hline 28 & BFREN & NRoFF & NRoFF & NRoFF \\
\hline 29 & BRİSA & RoFF & RoFF & RoFF \\
\hline 30 & BURCE & RoFF & RoFF & RoFF \\
\hline 31 & BURVA & RoFF & RoFF & RoFF \\
\hline 32 & BUCIM & RoFF & RoFF & RoFF \\
\hline 33 & CCOLA & RoFF & RoFF & RoFF \\
\hline 34 & CELHA & RoFF & RoFF & RoFF \\
\hline 35 & CEMAS & RoFF & RoFF & RoFF \\
\hline 36 & CEMTS & NRoFF & NRoFF & NRoFF \\
\hline 37 & CMBTN & RoFF & RoFF & RoFF \\
\hline 38 & CMENT & RoFF & RoFF & RoFF \\
\hline 39 & CIMSA & RoFF & RoFF & RoFF \\
\hline 40 & CUSAN & RoFF & RoFF & RoFF \\
\hline 41 & DAGI & RoFF & RoFF & RoFF \\
\hline 42 & DARDL & RoFF & RoFF & RoFF \\
\hline 43 & DMSAS & RoFF & RoFF & RoFF \\
\hline 44 & DERIM & RoFF & RoFF & RoFF \\
\hline 45 & DESA & RoFF & RoFF & RoFF \\
\hline 46 & DEVA & RoFF & NRoFF & RoFF \\
\hline 47 & DIRIT & RoFF & RoFF & RoFF \\
\hline 48 & DİTAS & RoFF & RoFF & RoFF \\
\hline 49 & DOBUR & NRoFF & RoFF & NRoFF \\
\hline 50 & DGKLB & RoFF & RoFF & RoFF \\
\hline 51 & DOGUB & RoFF & RoFF & RoFF \\
\hline 52 & DOKTA & RoFF & RoFF & RoFF \\
\hline 53 & DURDO & RoFF & RoFF & RoFF \\
\hline 54 & DYOBY & RoFF & RoFF & RoFF \\
\hline 55 & EGEEN & NRoFF & NRoFF & NRoFF \\
\hline 56 & EGGUB & RoFF & RoFF & RoFF \\
\hline 57 & EGPRO & RoFF & RoFF & RoFF \\
\hline 58 & $\begin{array}{l}\text { EGESER } \\
\end{array}$ & RoFF & RoFF & NRoFF \\
\hline 59 & EPLAS & RoFF & NRoFF & RoFF \\
\hline 60 & EKİZ & RoFF & RoFF & RoFF \\
\hline 61 & EMKEL & RoFF & RoFF & RoFF \\
\hline 62 & EMNIS & RoFF & RoFF & RoFF \\
\hline 63 & ERBOS & NRoFF & NRoFF & NRoFF \\
\hline 64 & EREGL & RoFF & RoFF & RoFF \\
\hline 65 & ERSU & RoFF & RoFF & RoFF \\
\hline 66 & FMZIP & NRoFF & RoFF & NRoFF \\
\hline 67 & FROTO & RoFF & RoFF & RoFF \\
\hline 68 & FORMT & RoFF & RoFF & RoFF \\
\hline 69 & FRIGO & RoFF & RoFF & RoFF \\
\hline 70 & GEDZA & NRoFF & NRoFF & NRoFF \\
\hline 71 & GENTS & RoFF & NRoFF & NRoFF \\
\hline 72 & GEREL & RoFF & RoFF & RoFF \\
\hline 73 & GOODY & RoFF & RoFF & RoFF \\
\hline 74 & GOLTS & RoFF & RoFF & RoFF \\
\hline 75 & GUBRF & RoFF & RoFF & RoFF \\
\hline 76 & HATEK & RoFF & RoFF & RoFF \\
\hline 77 & HEKTS & NRoFF & NRoFF & RoFF \\
\hline 78 & HURGZ & RoFF & RoFF & RoFF \\
\hline 79 & IHEVA & RoFF & RoFF & RoFF \\
\hline 80 & IHGZT & RoFF & RoFF & RoFF \\
\hline 81 & ISDMR & RoFF & RoFF & NRoFF \\
\hline 82 & IZDMC & RoFF & RoFF & RoFF \\
\hline 83 & JANTS & NRoFF & RoFF & NRoFF \\
\hline 84 & KAPLM & RoFF & RoFF & RoFF \\
\hline 85 & KARDMD & RoFF & RoFF & RoFF \\
\hline 86 & KARSN & RoFF & RoFF & RoFF \\
\hline 87 & KRTEK & RoFF & RoFF & RoFF \\
\hline 88 & KARTN & NRoFF & RoFF & NRoFF \\
\hline 89 & KATMR & RoFF & RoFF & RoFF \\
\hline 90 & KENT & RoFF & RoFF & RoFF \\
\hline 91 & KERVT & RoFF & RoFF & RoFF \\
\hline 92 & KLMSN & RoFF & RoFF & RoFF \\
\hline 93 & KONFRT & RoFF & RoFF & NRoFF \\
\hline 94 & KONYA & RoFF & RoFF & RoFF \\
\hline 95 & KORDS & RoFF & RoFF & RoFF \\
\hline 96 & KRSTL & RoFF & RoFF & RoFF \\
\hline 97 & KUTPO & NRoFF & NRoFF & NRoFF \\
\hline 98 & LUKSK & RoFF & RoFF & RoFF \\
\hline 99 & MAKTK & NRoFF & RoFF & NRoFF \\
\hline 100 & MRSHL & RoFF & RoFF & RoFF \\
\hline 101 & MNDRS & RoFF & RoFF & RoFF \\
\hline 102 & OLMK & RoFF & RoFF & RoFF \\
\hline 103 & TIRE & RoFF & RoFF & RoFF \\
\hline 104 & NİBAS & RoFF & RoFF & RoFF \\
\hline 105 & NUHCM & RoFF & RoFF & RoFF \\
\hline 106 & OTKAR & NRoFF & RoFF & NRoFF \\
\hline 107 & OYLUM & RoFF & RoFF & RoFF \\
\hline 108 & OZBAL & RoFF & RoFF & RoFF \\
\hline 109 & PARSN & RoFF & RoFF & RoFF \\
\hline
\end{tabular}

PAGE 98| Journal of Corporate Governance, Insurance, and Risk Management | 2021, VOL. 8, Series. 2 


\begin{tabular}{|c|c|c|c|c|c|c|c|c|c|}
\hline 110 & PENGD & RoFF & RoFF & RoFF & RoFF & RoFF & RoFF & RoFF & RoFF \\
\hline 111 & PETKM & RoFF & RoFF & RoFF & RoFF & RoFF & RoFF & RoFF & RoFF \\
\hline 112 & PETUN & RoFF & RoFF & RoFF & RoFF & RoFF & RoFF & RoFF & RoFF \\
\hline 113 & PINSU & RoFF & RoFF & RoFF & RoFF & RoFF & RoFF & RoFF & RoFF \\
\hline 114 & PNSUT & RoFF & RoFF & RoFF & RoFF & RoFF & RoFF & RoFF & RoFF \\
\hline 115 & PRZMA & RoFF & RoFF & RoFF & RoFF & RoFF & RoFF & RoFF & RoFF \\
\hline 116 & ROYAL & RoFF & RoFF & RoFF & RoFF & RoFF & RoFF & RoFF & RoFF \\
\hline 117 & RTLAB & RoFF & NRoFF & NRoFF & NRoFF & RoFF & NRoFF & RoFF & NRoFF \\
\hline 118 & SANFM & RoFF & RoFF & RoFF & RoFF & RoFF & RoFF & RoFF & RoFF \\
\hline 119 & SAMAT & RoFF & RoFF & RoFF & RoFF & RoFF & RoFF & RoFF & RoFF \\
\hline 120 & SARKY & RoFF & RoFF & RoFF & RoFF & RoFF & RoFF & RoFF & RoFF \\
\hline 121 & SAYAS & RoFF & NRoFF & RoFF & NRoFF & RoFF & RoFF & RoFF & NRoFF \\
\hline 122 & SASA & RoFF & RoFF & RoFF & RoFF & RoFF & RoFF & RoFF & RoFF \\
\hline 123 & SEKUR & RoFF & RoFF & RoFF & RoFF & RoFF & RoFF & RoFF & RoFF \\
\hline 124 & SELGD & RoFF & RoFF & RoFF & RoFF & RoFF & RoFF & NRoFF & RoFF \\
\hline 125 & $\begin{array}{l}\text { SILVR } \\
\end{array}$ & RoFF & RoFF & RoFF & RoFF & RoFF & RoFF & NRoFF & RoFF \\
\hline 126 & SKTAS & RoFF & RoFF & RoFF & RoFF & RoFF & RoFF & NRoFF & RoFF \\
\hline 127 & SNPAM & RoFF & RoFF & RoFF & RoFF & RoFF & RoFF & NRoFF & RoFF \\
\hline 128 & TATGD & NRoFF & NRoFF & NRoFF & NRoFF & NRoFF & NRoFF & NRoFF & RoFF \\
\hline 129 & TMPOL & RoFF & RoFF & RoFF & RoFF & RoFF & RoFF & NRoFF & RoFF \\
\hline 130 & TOASO & RoFF & RoFF & RoFF & RoFF & RoFF & RoFF & RoFF & RoFF \\
\hline 131 & TUCLK & RoFF & RoFF & RoFF & RoFF & RoFF & RoFF & RoFF & RoFF \\
\hline 132 & TUKAS & RoFF & RoFF & RoFF & RoFF & RoFF & RoFF & RoFF & RoFF \\
\hline 133 & TMSN & RoFF & RoFF & RoFF & RoFF & RoFF & RoFF & RoFF & RoFF \\
\hline 134 & TUPRS & RoFF & RoFF & RoFF & RoFF & RoFF & RoFF & RoFF & RoFF \\
\hline 135 & PRKAB & RoFF & RoFF & RoFF & RoFF & RoFF & RoFF & RoFF & RoFF \\
\hline 136 & TTRAK & RoFF & RoFF & RoFF & RoFF & RoFF & NRoFF & RoFF & NRoFF \\
\hline 137 & TBORG & RoFF & RoFF & RoFF & RoFF & NRoFF & NRoFF & NRoFF & NRoFF \\
\hline 138 & ULUSE & NRoFF & NRoFF & NRoFF & NRoFF & NRoFF & NRoFF & NRoFF & NRoFF \\
\hline 139 & ULUUN & RoFF & RoFF & RoFF & RoFF & RoFF & RoFF & RoFF & RoFF \\
\hline 140 & USAK & RoFF & RoFF & RoFF & RoFF & RoFF & RoFF & RoFF & RoFF \\
\hline 141 & ULKER & NRoFF & RoFF & RoFF & RoFF & RoFF & RoFF & RoFF & NRoFF \\
\hline 142 & VESBE & RoFF & RoFF & RoFF & RoFF & RoFF & RoFF & RoFF & RoFF \\
\hline 143 & VESTL & RoFF & RoFF & RoFF & RoFF & RoFF & RoFF & RoFF & RoFF \\
\hline 144 & VKING & RoFF & RoFF & RoFF & RoFF & RoFF & RoFF & RoFF & RoFF \\
\hline 145 & YATAS & NRoFF & NRoFF & NRoFF & RoFF & NRoFF & NRoFF & NRoFF & NRoFF \\
\hline 146 & YUNSA & RoFF & RoFF & RoFF & RoFF & RoFF & RoFF & RoFF & RoFF \\
\hline \multirow{2}{*}{ TOTAL } & NO RISK OF FAILURE & 20 & 20 & 25 & 23 & 21 & 36 & 27 & 38 \\
\hline & RISK OF FINANCIAL FAILURE & 126 & 126 & 121 & 123 & 125 & 110 & 119 & 108 \\
\hline
\end{tabular}

Source: Authors' Compilation

When Table 7 is examined, the period of 2020/12 is seen as the period with the highest success, and the Springate scores show a total of 38 companies being successful. The least successful periods were experienced in 2019/3 and 2019/6. Only 20 companies scored successfully within those periods. In Table 7, companies that are financially successful in all quarters in terms of Springate scores as of 2019-2020 and quarterly periods are as follows: ALCAR, ALKIM, CEMTS, KUTPO, ULUSE. The companies that failed in all periods are ADEL, AFYON, AKCNS, ATEKS, AKSA, AEFES, ARSAN, ASUZU, AVOD, AYGAZ, BAGFS, BAKAB, BTCIM, BRKO, BRMEN BRSAN, BOSSA, BRISA, BURCE, CEMAS, CMBTN, CMENT, CIMSA, CUSAN, DAGI, DARDL, DERIM, DIRIT, DGKLB, DOGUB, DOKTA, DURDO, DYOBY, EGGUB, EGPRO, EKIZ, EMKEL, EREGL, ERSU, FORMT, GEREL, GUBRF, IZDMC, KAPLM, KARDMD, KARSN, KENT, KLMSN, KORDS, KRSTL, LUKSK, MRSHL, MNDRS, OLMK, TIRE, NIBAS, NUHCM, OYLUM, OZBAL, PARSN, PENGD, PETKM, PETUN, PINSU, PNSUT, PRZMA, ROYAL, SANFM, SAMAT, SARKY, TOASO, TUCLK, TUKAS, TMSN, TUPRS, PRKAB, ULUUN, USAK, VESBE, VESTL, VKING and YUNSA.

According to the formula in Table 3, the Taffler model's values of the BIST manufacturing companies within the scope of the research for the years 2019-2020 are given in Table 8.

Table 8: Taffler Model's Values of BIST Manufacturing Companies for 2019-2020

\begin{tabular}{|c|c|c|c|c|c|c|c|c|c|}
\hline \multirow{2}{*}{ No. } & \multirow{2}{*}{ Code } & \multicolumn{8}{|c|}{ Taffler } \\
\hline & & $2019 / 3$ & $2020 / 3$ & $2019 / 6$ & $2020 / 6$ & $2019 / 9$ & $2020 / 9$ & $2019 / 12$ & $2020 / 12$ \\
\hline 1 & ACSEL & 9,41 & 13,79 & 12,59 & 18,67 & 9,47 & 18,67 & 10,22 & 23,28 \\
\hline 2 & ADEL & 0,76 & $\begin{array}{l}-0,24 \\
\end{array}$ & $-0,67$ & $-0,64$ & $-0,62$ & $-0,80$ & 0,46 & $-1,28$ \\
\hline 3 & AFYON & $-1,77$ & $-2,50$ & $-1,82$ & 2,05 & $-1,59$ & 2,56 & $-2,23$ & 2,50 \\
\hline 4 & AKCNS & 0,23 & 0,35 & 0,41 & 1,96 & 1,07 & 3,00 & 2,18 & 2,47 \\
\hline 5 & ATEKS & 3,35 & 2,47 & 3,24 & 2,36 & 2,86 & 2,63 & 2,93 & 7,16 \\
\hline 6 & AKSA & 0,97 & 0,52 & 0,57 & 0,22 & 0,71 & 2,11 & 0,29 & 3,18 \\
\hline
\end{tabular}

PAGE 99| Journal of Corporate Governance, Insurance, and Risk Management |2021, VOL. 8, Series. 2 


\begin{tabular}{|c|c|c|c|c|}
\hline 7 & ALCAR & 8,34 & 7,13 & 6,21 \\
\hline 8 & ALKA & 2,54 & 7,03 & 6,39 \\
\hline 9 & ALKIM & 8,22 & 11,93 & 9,70 \\
\hline 10 & AEFES & 1,83 & 1,97 & 2,82 \\
\hline 11 & ARCLK & 1,23 & 1,41 & 1,09 \\
\hline 12 & ARSAN & 3,55 & 5,56 & 3,90 \\
\hline 13 & ASUZU & $-0,40$ & $-0,33$ & $-0,85$ \\
\hline 14 & AVOD & 1,08 & $-0,43$ & 1,15 \\
\hline 15 & AYGAZ & 0,68 & 0,17 & 2,39 \\
\hline 16 & BAGFS & 1,56 & 0,46 & 1,06 \\
\hline 17 & BAKAB & 1,32 & 1,61 & 1,23 \\
\hline 18 & BANVT & 1,00 & 1,96 & 4,02 \\
\hline 19 & BRKSN & 0,14 & 0,26 & 0,23 \\
\hline 20 & BLCYT & 6,31 & 14,16 & 7,72 \\
\hline 21 & BNTAS & 13,03 & 17,20 & 13,67 \\
\hline 22 & BSOKE & $-1,42$ & $-2,55$ & $-1,19$ \\
\hline 23 & BTCIM & $-0,21$ & $-1,41$ & $-0,19$ \\
\hline 24 & BRKO & 0,71 & 0,04 & 1,18 \\
\hline 25 & BRMEN & $-4,85$ & $-15,04$ & $-4,99$ \\
\hline 26 & BRSAN & 1,09 & 0,58 & 1,68 \\
\hline 27 & BOSSA & 0,78 & 0,39 & 0,80 \\
\hline 28 & BFREN & 14,76 & 10,86 & 9,69 \\
\hline 29 & BRİSA & 0,88 & $-0,36$ & 0,89 \\
\hline 30 & BURCE & $-0,37$ & 0,63 & $-0,26$ \\
\hline 31 & BURVA & 0,54 & $-0,15$ & 0,41 \\
\hline 32 & BUCIM & 8,62 & 9,04 & 9,10 \\
\hline 33 & CCOLA & 2,42 & 2,73 & 2,93 \\
\hline 34 & CELHA & $-3,28$ & $-4,07$ & $-3,07$ \\
\hline 35 & CEMAS & 2,41 & 8,07 & 2,22 \\
\hline 36 & CEMTS & 11,51 & 14,45 & 15,88 \\
\hline 37 & CMBTN & 0,86 & $-2,46$ & 0,04 \\
\hline 38 & CMENT & 2,92 & 1,18 & 3,25 \\
\hline 39 & CIMSA & 0,30 & $-0,35$ & $-0,35$ \\
\hline 40 & CUSAN & 1,05 & 2,75 & 2,21 \\
\hline 41 & DAGİ & 0,60 & 0,69 & 1,81 \\
\hline 42 & DARDL & $-26,09$ & $-5,45$ & $-26,42$ \\
\hline 43 & DMSAS & 2,55 & 1,70 & 1,31 \\
\hline 44 & DERIM & $-0,69$ & $-0,57$ & $-1,46$ \\
\hline 45 & DESA & $-0,45$ & $-1,33$ & $-0,44$ \\
\hline 46 & DEVA & 3,63 & 5,49 & 3,06 \\
\hline 47 & DIRIT & $-3,60$ & 1,47 & $-5,02$ \\
\hline 48 & DİTAS & 0,77 & $-1,74$ & 1,00 \\
\hline 49 & DOBUR & 2,53 & $\begin{array}{l}0,68 \\
\end{array}$ & 2,17 \\
\hline 50 & DGKLB & $-2,55$ & $-3,06$ & $-2,05$ \\
\hline 51 & DOGUB & $-3,35$ & 1,17 & 2,20 \\
\hline 52 & DOKTA & $-0,68$ & 0,06 & $-0,23$ \\
\hline 53 & DURDO & 0,25 & $-0,10$ & 1,00 \\
\hline 54 & DYOBY & $-2,56$ & $-2,80$ & $-2,74$ \\
\hline 55 & EGEEN & 18,29 & 11,32 & 14,06 \\
\hline 56 & EGGUB & 1,10 & $-0,32$ & 1,85 \\
\hline 57 & EGPRO & $-0,25$ & 0,77 & $-0,14$ \\
\hline 58 & $\begin{array}{l}\text { EGESER } \\
\end{array}$ & 7,11 & 5,25 & 6,17 \\
\hline 59 & EPLAS & 0,95 & 6,97 & 0,98 \\
\hline 60 & EKİZ & $\begin{array}{l}-4,03 \\
\end{array}$ & $\begin{array}{l}-2,04 \\
\end{array}$ & $\begin{array}{l}-4,66 \\
\end{array}$ \\
\hline 61 & EMKEL & $-2,00$ & $-0,94$ & $-1,33$ \\
\hline 62 & EMNIS & $-6,67$ & $\begin{array}{ll}-0,28 \\
\end{array}$ & 1,56 \\
\hline 63 & ERBOS & 8,33 & 6,69 & 6,95 \\
\hline 64 & EREGL & 5,54 & 6,01 & 8,06 \\
\hline 65 & ERSU & $\begin{array}{l}4,55 \\
\end{array}$ & 6,16 & 4,33 \\
\hline 66 & FMZİP & 27,25 & 22,72 & 38,86 \\
\hline 67 & FROTO & $\begin{array}{l}-0,78 \\
\end{array}$ & 0,17 & $\begin{array}{l}-0,33 \\
\end{array}$ \\
\hline 68 & FORMT & 2,84 & 0,35 & 2,65 \\
\hline 69 & FRİGO & 2,15 & 2,34 & 4,87 \\
\hline 70 & GEDZA & 9,46 & 9,49 & 9,21 \\
\hline 71 & GENTS & 6,30 & 8,73 & 6,99 \\
\hline 72 & GEREL & 2,58 & 1,09 & 0,38 \\
\hline 73 & GOODY & $-0,06$ & 1,26 & 0,55 \\
\hline 74 & GOLTS & 1,52 & 0,86 & 0,96 \\
\hline 75 & GUBRF & $-1,59$ & $-2,17$ & $-2,04$ \\
\hline 76 & HATEK & 2,59 & 2,01 & 1,47 \\
\hline 77 & HEKTS & 3,16 & 4,99 & 1,93 \\
\hline 78 & HURGZ & 4,57 & 3,55 & 3,42 \\
\hline 79 & IHEVA & 7,96 & 9,82 & 5,81 \\
\hline 80 & IHGZT & 7,01 & 0,73 & 5,22 \\
\hline 81 & ISDMR & 5,08 & 7,60 & 11,34 \\
\hline 82 & IZDMC & $-4,98$ & $-5,13$ & $-3,82$ \\
\hline 83 & JANTS & 5,65 & 4,22 & 6,48 \\
\hline 84 & KAPLM & $-1,96$ & $-1,50$ & $-2,36$ \\
\hline 85 & KARDMD & 1,46 & $-0,14$ & 2,17 \\
\hline 86 & KARSN & $-2,17$ & $\begin{array}{l}-0,85 \\
\end{array}$ & $-1,12$ \\
\hline 87 & KRTEK & 1,26 & 0,75 & 1,31 \\
\hline 88 & KARTN & 15,23 & 13,05 & 13,76 \\
\hline 89 & $\begin{array}{l}\text { KATMR } \\
\end{array}$ & $-1,10$ & 2,20 & 2,28 \\
\hline 90 & KENT & 1,60 & 2,04 & 1,13 \\
\hline 91 & KERVT & 3,03 & 3,65 & 4,28 \\
\hline 92 & KLMSN & 2,22 & 0,39 & 2,26 \\
\hline 93 & KONFRT & 5,20 & 1,31 & 7,94 \\
\hline 94 & KONYA & 8,06 & 5,84 & 7,98 \\
\hline 95 & KORDS & 3,25 & 2,64 & 2,91 \\
\hline 96 & KRSTL & 9,01 & 7,96 & 6,60 \\
\hline 97 & KUTPO & 6,36 & 6,18 & 6,67 \\
\hline 98 & LUKSK & 3,56 & 2,93 & 4,45 \\
\hline 99 & MAKTK & 6,06 & 7,14 & 6,86 \\
\hline 100 & MRSHL & $-2,60$ & $-2,23$ & $-2,77$ \\
\hline
\end{tabular}

PAGE 100| Journal of Corporate Governance, Insurance, and Risk Management | 2021, VOL. 8, Series. 2 


\begin{tabular}{|c|c|c|c|c|c|c|c|c|c|}
\hline 101 & MNDRS & $-1,44$ & $-2,81$ & $-1,85$ & $-1,39$ & $-0,45$ & $-2,19$ & $-1,82$ & 2,17 \\
\hline 102 & OLMK & $-1,20$ & $-0,07$ & $-2,59$ & 0,07 & $-1,30$ & 0,03 & $-2,72$ & 0,41 \\
\hline 103 & TIRE & $-1,54$ & $-1,37$ & $-2,00$ & $-1,30$ & $-1,43$ & $-1,26$ & $-1,39$ & $-0,70$ \\
\hline 104 & NIBAS & 3,68 & 6,21 & 3,05 & 4,56 & 3,78 & 2,91 & 10,49 & $-3,13$ \\
\hline 105 & NUHCM & 2,91 & 6,35 & 3,09 & 9,06 & 3,34 & 8,06 & 5,13 & 7,32 \\
\hline 106 & OTKAR & $-0,44$ & 1,10 & 2,88 & 2,09 & 3,98 & 1,33 & 2,12 & 2,59 \\
\hline 107 & OYLUM & 1,23 & 3,81 & 2,00 & 4,57 & 2,02 & 5,23 & 1,99 & 3,92 \\
\hline 108 & OZBAL & $-8,01$ & $-0,97$ & $-8,34$ & $-0,47$ & $-8,33$ & 1,40 & $-4,50$ & $-1,74$ \\
\hline 109 & PARSN & 1,61 & 2,31 & 1,05 & 0,88 & 0,51 & 2,32 & 0,96 & 3,52 \\
\hline 110 & PENGD & $-3,00$ & $-0,90$ & $-3,34$ & $-1,42$ & $-2,71$ & 3,31 & $-0,48$ & 4,65 \\
\hline 111 & PETKM & 3,24 & 2,41 & 3,17 & 3,01 & 2,73 & 2,82 & 2,27 & 4,00 \\
\hline 112 & PETUN & 3,67 & 5,09 & 4,99 & 4,73 & 5,85 & 5,07 & 6,02 & 6,16 \\
\hline 113 & PINSU & $-3,78$ & $-3,61$ & $-3,99$ & $-3,65$ & $-2,88$ & $-3,34$ & $-2,67$ & $-3,68$ \\
\hline 114 & PNSUT & 2,00 & 2,53 & 1,48 & 1,84 & 1,53 & 1,60 & 2,29 & 1,47 \\
\hline 115 & PRZMA & 7,65 & 20,13 & 10,31 & 10,38 & 10,53 & 3,77 & 11,24 & 14,72 \\
\hline 116 & ROYAL & $-3,66$ & $-0,62$ & $-1,69$ & $-1,29$ & $-1,88$ & $-0,41$ & $-2,47$ & 0,34 \\
\hline 117 & RTLAB & 19,13 & 16,33 & 20,10 & 23,52 & 13,09 & 12,23 & 9,70 & 9,71 \\
\hline 118 & SANFM & 2,59 & 3,66 & 3,88 & 4,02 & 3,21 & 5,26 & 16,58 & 6,14 \\
\hline 119 & SAMAT & $-1,22$ & $-2,06$ & $-1,53$ & 0,17 & $-1,35$ & 0,36 & $-4,01$ & 0,74 \\
\hline 120 & SARKY & 0,50 & 1,12 & $-0,37$ & 1,46 & $-0,25$ & 1,71 & $-0,13$ & 1,11 \\
\hline 121 & SAYAS & 0,75 & 0,74 & 1,28 & 4,38 & 1,64 & 0,74 & $-0,29$ & 3,51 \\
\hline 122 & SASA & 1,30 & $-1,60$ & 0,34 & 0,09 & 0,07 & $-2,17$ & $-1,41$ & 0,89 \\
\hline 123 & SEKUR & 0,51 & 0,97 & 1,02 & 0,54 & 1,30 & 0,66 & 1,32 & 0,38 \\
\hline 124 & SELGD & 7,37 & 7,60 & 7,48 & 7,07 & 7,63 & 10,40 & 14,97 & 15,64 \\
\hline 125 & SILVR & $-2,56$ & $-1,80$ & $-1,01$ & $-1,94$ & $-1,76$ & $-2,37$ & $-1,44$ & $-1,65$ \\
\hline 126 & SKTAS & 0,29 & $-2,76$ & $-1,18$ & $-3,07$ & $-0,77$ & $-0,97$ & $-1,52$ & $-0,44$ \\
\hline 127 & SNPAM & 4,72 & 5,51 & 5,73 & 1,70 & 3,90 & 2,69 & 14,48 & 8,90 \\
\hline 128 & $\begin{array}{l}\text { TATGD } \\
\end{array}$ & 5,45 & 7,19 & 5,66 & 5,72 & 3,99 & 3,28 & 5,45 & 4,71 \\
\hline 129 & TMPOL & 2,18 & 0,07 & 0,65 & 0,25 & 0,77 & 0,31 & 1,34 & 0,57 \\
\hline 130 & TOASO & 0,23 & 0,69 & 0,12 & 0,69 & 0,75 & 0,43 & 1,04 & 0,30 \\
\hline 131 & TUCLK & 0,86 & 0,92 & 0,40 & 0,56 & 1,50 & 0,42 & $-0,59$ & 0,51 \\
\hline 132 & TUKAS & $-0,01$ & 1,84 & 0,51 & 1,64 & 0,00 & 1,82 & 1,39 & 2,51 \\
\hline 133 & TMSN & 1,27 & 1,93 & 0,79 & 2,67 & 1,57 & 3,14 & 2,19 & 4,69 \\
\hline 134 & TUPRS & $-0,30$ & $-1,93$ & 0,09 & $-0,53$ & $-0,43$ & $-0,01$ & $-0,82$ & $-0,26$ \\
\hline 135 & PRKAB & 0,10 & 0,84 & $-0,17$ & 0,98 & 0,61 & 0,47 & 1,35 & 0,43 \\
\hline 136 & TTRAK & 0,23 & 1,00 & $-0,43$ & 2,26 & 0,65 & 3,03 & 2,18 & 5,00 \\
\hline 137 & TBORG & $-0,12$ & 1,08 & 0,04 & 1,74 & 3,50 & 4,99 & 3,79 & 3,13 \\
\hline 138 & ULUSE & 23,87 & 7,39 & 14,81 & 8,81 & 14,54 & 8,61 & 11,81 & 3,50 \\
\hline 139 & ULUUN & $-0,32$ & $-0,79$ & $-0,11$ & $-0,50$ & $-0,64$ & $-0,71$ & $-0,68$ & $-0,50$ \\
\hline 140 & USAK & $-0,61$ & $-0,13$ & $-0,68$ & 0,86 & $-0,97$ & 1,39 & $-0,11$ & 0,85 \\
\hline 141 & ULKER & 6,82 & $-0,17$ & 2,56 & 4,30 & 3,19 & 3,65 & 1,00 & 5,87 \\
\hline 142 & VESBE & 0,18 & 0,01 & $-0,28$ & 0,82 & $-0,31$ & 0,65 & $-0,20$ & 2,35 \\
\hline 143 & VESTL & $-3,58$ & $-2,55$ & $-3,39$ & $-2,39$ & $-3,39$ & $-2,27$ & $-3,24$ & $-1,12$ \\
\hline 144 & VKING & $-4,04$ & $-3,95$ & $-4,92$ & $-4,20$ & $-5,23$ & $-5,11$ & $-4,40$ & $-5,55$ \\
\hline 145 & YATAS & 1,57 & 2,16 & 2,56 & 2,01 & 2,52 & 3,98 & 1,99 & 3,60 \\
\hline 146 & $\begin{array}{l}\text { YUNSA } \\
\end{array}$ & $-1,76$ & 0,09 & $-1,59$ & 0,07 & $-2,25$ & 1,14 & $-1,11$ & 0,78 \\
\hline
\end{tabular}

Source: Authors' Compilation

The companies' determination within the research scope according to the Taffler values in Table 8 for the years 2019-2020 according to the threshold values (Table 3) is given in Table 9. RoFF (Risk of Financial Failure) was used for companies with a risk of financial failure and NRoFF (No Risk of Financial Failure) for companies with no risk of financial failure.

Table 9: Financial Status According to Taffler Model's Values of Manufacturing Companies for 2019-2020

\begin{tabular}{|c|c|c|c|c|c|c|c|c|c|}
\hline \multirow{2}{*}{ No. } & \multirow{2}{*}{ Code } & \multicolumn{8}{|c|}{ Taffler-Financial Status } \\
\hline & & $2019 / 3$ & $2020 / 3$ & $2019 / 6$ & $2020 / 6$ & $2019 / 9$ & $2020 / 9$ & $2019 / 12$ & $2020 / 12$ \\
\hline 1 & ACSEL & NRoFF & NRoFF & NRoFF & NRoFF & NRoFF & NRoFF & NRoFF & NRoFF \\
\hline 2 & ADEL & NRoFF & RoFF & RoFF & RoFF & RoFF & RoFF & NRoFF & RoFF \\
\hline 3 & AFYON & RoFF & RoFF & RoFF & NRoFF & RoFF & NRoFF & RoFF & NRoFF \\
\hline 4 & AKCNS & RoFF & NRoFF & NRoFF & NRoFF & NRoFF & NRoFF & NRoFF & NRoFF \\
\hline 5 & ATEKS & NRoFF & NRoFF & NRoFF & NRoFF & NRoFF & NRoFF & NRoFF & NRoFF \\
\hline 6 & AKSA & NRoFF & NRoFF & NRoFF & RoFF & NRoFF & NRoFF & RoFF & NRoFF \\
\hline 7 & ALCAR & NRoFF & NRoFF & NRoFF & NRoFF & NRoFF & NRoFF & NRoFF & NRoFF \\
\hline 8 & ALKA & NRoFF & NRoFF & NRoFF & NRoFF & NRoFF & NRoFF & NRoFF & NRoFF \\
\hline 9 & ALKİM & NRoFF & NRoFF & NRoFF & NRoFF & NRoFF & NRoFF & NRoFF & NRoFF \\
\hline 10 & AEFES & NRoFF & NRoFF & NRoFF & NRoFF & NRoFF & NRoFF & NRoFF & NRoFF \\
\hline 11 & ARCLK & NRoFF & NRoFF & NRoFF & NRoFF & NRoFF & NRoFF & NRoFF & NRoFF \\
\hline 12 & ARSAN & NRoFF & NRoFF & NRoFF & NRoFF & NRoFF & NRoFF & NRoFF & NRoFF \\
\hline 13 & ASUZU & RoFF & RoFF & RoFF & RoFF & RoFF & RoFF & RoFF & RoFF \\
\hline 14 & AVOD & NRoFF & RoFF & NRoFF & RoFF & NRoFF & RoFF & RoFF & RoFF \\
\hline 15 & AYGAZ & NRoFF & NRoFF & NRoFF & NRoFF & NRoFF & NRoFF & NRoFF & NRoFF \\
\hline 16 & BAGFS & NRoFF & NRoFF & NRoFF & RoFF & NRoFF & RoFF & RoFF & NRoFF \\
\hline 17 & BAKAB & NRoFF & NRoFF & NRoFF & NRoFF & NRoFF & NRoFF & NRoFF & NRoFF \\
\hline 18 & BANVT & NRoFF & NRoFF & NRoFF & NRoFF & NRoFF & NRoFF & NRoFF & RoFF \\
\hline 19 & BRKSN & RoFF & RoFF & RoFF & NRoFF & RoFF & NRoFF & NRoFF & NRoFF \\
\hline 20 & BLCYT & NRoFF & NRoFF & NRoFF & NRoFF & NRoFF & NRoFF & NRoFF & NRoFF \\
\hline 21 & BNTAS & NRoFF & NRoFF & NRoFF & NRoFF & NRoFF & NRoFF & NRoFF & NRoFF \\
\hline 22 & BSOKE & RoFF & RoFF & RoFF & RoFF & NRoFF & RoFF & RoFF & RoFF \\
\hline 23 & BTCIM & RoFF & RoFF & RoFF & RoFF & NRoFF & RoFF & RoFF & RoFF \\
\hline 24 & BRKO & NRoFF & RoFF & NRoFF & RoFF & RoFF & NRoFF & RoFF & NRoFF \\
\hline 25 & BRMEN & RoFF & RoFF & RoFF & RoFF & RoFF & RoFF & RoFF & RoFF \\
\hline 26 & BRSAN & NRoFF & NRoFF & NRoFF & NRoFF & NRoFF & NRoFF & NRoFF & NRoFF \\
\hline 27 & BOSSA & NRoFF & NRoFF & NRoFF & RoFF & NRoFF & RoFF & NRoFF & NRoFF \\
\hline 28 & BFREN & NRoFF & NRoFF & NRoFF & NRoFF & NRoFF & NRoFF & NRoFF & NRoFF \\
\hline 29 & BRİSA & NRoFF & RoFF & NRoFF & RoFF & NRoFF & NRoFF & RoFF & NRoFF \\
\hline 30 & BURCE & RoFF & NRoFF & RoFF & NRoFF & RoFF & NRoFF & NRoFF & NRoFF \\
\hline
\end{tabular}

PAGE 101| Journal of Corporate Governance, Insurance, and Risk Management |2021, VOL. 8, Series. 2 


\begin{tabular}{|c|c|c|c|c|}
\hline 31 & BURVA & NRoFF & RoFF & NRoFF \\
\hline 32 & BUCIM & NRoFF & NRoFF & NRoFF \\
\hline 33 & CCOLA & NRoFF & NRoFF & NRoFF \\
\hline 34 & CELHA & RoFF & RoFF & RoFF \\
\hline 35 & CEMAS & NRoFF & NRoFF & NRoFF \\
\hline 36 & CEMTS & NRoFF & NRoFF & NRoFF \\
\hline 37 & CMBTN & NRoFF & RoFF & RoFF \\
\hline 38 & CMENT & NRoFF & NRoFF & NRoFF \\
\hline 39 & CIMSA & RoFF & RoFF & RoFF \\
\hline 40 & CUSAN & NRoFF & NRoFF & NRoFF \\
\hline 41 & DAGİ & NRoFF & NRoFF & NRoFF \\
\hline 42 & DARDL & RoFF & RoFF & RoFF \\
\hline 43 & DMSAS & NRoFF & NRoFF & NRoFF \\
\hline 44 & DERIM & RoFF & RoFF & RoFF \\
\hline 45 & DESA & NRoFF & NRoFF & NRoFF \\
\hline 46 & DEVA & NRoFF & NRoFF & NRoFF \\
\hline 47 & DIRIT & RoFF & NRoFF & RoFF \\
\hline 48 & DITAS & NRoFF & RoFF & NRoFF \\
\hline 49 & DOBUR & NRoFF & NRoFF & NRoFF \\
\hline 50 & DGKLB & RoFF & RoFF & RoFF \\
\hline 51 & DOGUB & NRoFF & NRoFF & NRoFF \\
\hline 52 & DOKTA & RoFF & RoFF & RoFF \\
\hline 53 & $\begin{array}{l}\text { DURDO } \\
\end{array}$ & RoFF & RoFF & NRoFF \\
\hline 54 & DYOBY & RoFF & RoFF & RoFF \\
\hline 55 & EGEEN & NRoFF & NRoFF & NRoFF \\
\hline 56 & EGGUB & NRoFF & RoFF & NRoFF \\
\hline 57 & EGPRO & RoFF & NRoFF & RoFF \\
\hline 58 & EGESER & NRoFF & NRoFF & NRoFF \\
\hline 59 & EPLAS & NRoFF & NRoFF & NRoFF \\
\hline 60 & EKİZ & RoFF & RoFF & RoFF \\
\hline 61 & EMKEL & RoFF & RoFF & RoFF \\
\hline 62 & EMNIS & RoFF & RoFF & NRoFF \\
\hline 63 & ERBOS & NRoFF & NRoFF & NRoFF \\
\hline 64 & EREGL & NRoFF & NRoFF & NRoFF \\
\hline 65 & ERSU & NRoFF & NRoFF & NRoFF \\
\hline 66 & FMZİP & NRoFF & NRoFF & NRoFF \\
\hline 67 & FROTO & RoFF & RoFF & RoFF \\
\hline 68 & FORMT & NRoFF & NRoFF & NRoFF \\
\hline 69 & FRİGO & NRoFF & NRoFF & NRoFF \\
\hline 70 & GEDZA & NRoFF & NRoFF & NRoFF \\
\hline 71 & GENTS & NRoFF & NRoFF & NRoFF \\
\hline 72 & GEREL & NRoFF & NRoFF & NRoFF \\
\hline 73 & GOODY & RoFF & NRoFF & NRoFF \\
\hline 74 & GOLTS & NRoFF & NRoFF & NRoFF \\
\hline 75 & GUBRF & RoFF & RoFF & RoFF \\
\hline 76 & $\begin{array}{l}\text { HATEK } \\
\end{array}$ & NRoFF & NRoFF & NRoFF \\
\hline 77 & HEKTS & NRoFF & NRoFF & NRoFF \\
\hline 78 & HURGZ & NRoFF & NRoFF & NRoFF \\
\hline 79 & IHEVA & NRoFF & NRoFF & NRoFF \\
\hline 80 & IHGZT & NRoFF & NRoFF & NRoFF \\
\hline 81 & ISDMR & NRoFF & NRoFF & NRoFF \\
\hline 82 & IZDMC & RoFF & RoFF & RoFF \\
\hline 83 & JANTS & NRoFF & NRoFF & NRoFF \\
\hline 84 & KAPLM & RoFF & RoFF & RoFF \\
\hline 85 & KARDMD & NRoFF & RoFF & NRoFF \\
\hline 86 & KARSN & RoFF & RoFF & RoFF \\
\hline 87 & KRTEK & NRoFF & NRoFF & NRoFF \\
\hline 88 & KARTN & NRoFF & NRoFF & NRoFF \\
\hline 89 & KATMR & RoFF & NRoFF & NRoFF \\
\hline 90 & KENT & NRoFF & NRoFF & NRoFF \\
\hline 91 & KERVT & NRoFF & NRoFF & NRoFF \\
\hline 92 & KLMSN & NRoFF & NRoFF & NRoFF \\
\hline 93 & KONFRT & NRoFF & NRoFF & NRoFF \\
\hline 94 & KONYA & NRoFF & NRoFF & NRoFF \\
\hline 95 & KORDS & NRoFF & NRoFF & NRoFF \\
\hline 96 & KRSTL & NRoFF & NRoFF & NRoFF \\
\hline 97 & KUTPO & NRoFF & NRoFF & NRoFF \\
\hline 98 & LUKSK & NRoFF & NRoFF & NRoFF \\
\hline 99 & MAKTK & NRoFF & NRoFF & NRoFF \\
\hline 100 & MRSHL & RoFF & RoFF & RoFF \\
\hline 101 & MNDRS & RoFF & RoFF & RoFF \\
\hline 102 & OLMK & RoFF & RoFF & RoFF \\
\hline 103 & TiRE & RoFF & RoFF & RoFF \\
\hline 104 & NIBAS & NRoFF & NRoFF & NRoFF \\
\hline 105 & NUHCM & NRoFF & NRoFF & NRoFF \\
\hline 106 & $\begin{array}{l}\text { OTKAR } \\
\end{array}$ & NRoFF & NRoFF & NRoFF \\
\hline 107 & OYLUM & NRoFF & NRoFF & NRoFF \\
\hline 108 & OZBAL & RoFF & RoFF & RoFF \\
\hline 109 & PARSN & NRoFF & NRoFF & NRoFF \\
\hline 110 & PENGD & RoFF & RoFF & RoFF \\
\hline 111 & PETKM & NRoFF & NRoFF & NRoFF \\
\hline 112 & PETUN & NRoFF & NRoFF & NRoFF \\
\hline 113 & PINSU & RoFF & RoFF & RoFF \\
\hline 114 & $\begin{array}{l}\text { PNSUT } \\
\end{array}$ & NRoFF & NRoFF & NRoFF \\
\hline 115 & PRZMA & NRoFF & NRoFF & NRoFF \\
\hline 116 & ROYAL & RoFF & RoFF & RoFF \\
\hline 117 & RTLAB & NRoFF & NRoFF & NRoFF \\
\hline 118 & SANFM & NRoFF & NRoFF & NRoFF \\
\hline 119 & $\begin{array}{l}\text { SAMAT } \\
\end{array}$ & RoFF & RoFF & RoFF \\
\hline 120 & SARKY & RoFF & NRoFF & RoFF \\
\hline 121 & SAYAS & NRoFF & NRoFF & NRoFF \\
\hline 122 & SASA & NRoFF & RoFF & NRoFF \\
\hline 123 & SEKUR & NRoFF & NRoFF & NRoFF \\
\hline 124 & SELGD & NRoFF & NRoFF & NRoFF \\
\hline
\end{tabular}

PAGE 102| Journal of Corporate Governance, Insurance, and Risk Management | 2021, VOL. 8, Series. 2 


\begin{tabular}{|c|c|c|c|c|c|c|c|c|c|}
\hline 125 & SILVR & RoFF & RoFF & RoFF & RoFF & RoFF & RoFF & RoFF & RoFF \\
\hline 126 & SKTAS & RoFF & RoFF & RoFF & RoFF & RoFF & RoFF & RoFF & RoFF \\
\hline 127 & SNPAM & NRoFF & NRoFF & NRoFF & NRoFF & NRoFF & NRoFF & NRoFF & NRoFF \\
\hline 128 & TATGD & NRoFF & NRoFF & NRoFF & NRoFF & NRoFF & NRoFF & NRoFF & NRoFF \\
\hline 129 & TMPOL & NRoFF & RoFF & NRoFF & RoFF & NRoFF & NRoFF & NRoFF & NRoFF \\
\hline 130 & TOASO & RoFF & NRoFF & RoFF & NRoFF & NRoFF & NRoFF & NRoFF & RoFF \\
\hline 131 & TUCLK & NRoFF & NRoFF & NRoFF & NRoFF & NRoFF & NRoFF & RoFF & NRoFF \\
\hline 132 & TUKAS & RoFF & NRoFF & NRoFF & NRoFF & RoFF & $\mathrm{NRoFF}$ & NRoFF & NRoFF \\
\hline 133 & TMSN & NRoFF & NRoFF & NRoFF & NRoFF & NRoFF & NRoFF & NRoFF & NRoFF \\
\hline 134 & TUPRS & RoFF & RoFF & RoFF & RoFF & RoFF & RoFF & RoFF & RoFF \\
\hline 135 & PRKAB & RoFF & NRoFF & RoFF & NRoFF & NRoFF & NRoFF & NRoFF & NRoFF \\
\hline 136 & $\begin{array}{l}\text { TTRAK } \\
\end{array}$ & RoFF & NRoFF & RoFF & NRoFF & NRoFF & NRoFF & NRoFF & NRoFF \\
\hline 137 & TBORG & RoFF & NRoFF & RoFF & NRoFF & NRoFF & NRoFF & NRoFF & NRoFF \\
\hline 138 & ULUSE & NRoFF & NRoFF & NRoFF & NRoFF & NRoFF & NRoFF & NRoFF & NRoFF \\
\hline 139 & ULUUN & RoFF & RoFF & RoFF & RoFF & RoFF & RoFF & RoFF & RoFF \\
\hline 140 & USAK & RoFF & RoFF & RoFF & NRoFF & RoFF & NRoFF & RoFF & NRoFF \\
\hline 141 & ULKER & NRoFF & RoFF & NRoFF & NRoFF & NRoFF & NRoFF & NRoFF & NRoFF \\
\hline 142 & VESBE & RoFF & RoFF & RoFF & NRoFF & RoFF & NRoFF & RoFF & NRoFF \\
\hline 143 & VESTL & RoFF & RoFF & RoFF & RoFF & RoFF & RoFF & RoFF & RoFF \\
\hline 144 & VKING & RoFF & RoFF & RoFF & RoFF & RoFF & RoFF & RoFF & RoFF \\
\hline 145 & YATAS & NRoFF & $\mathrm{NRoFF}$ & NRoFF & NRoFF & NRoFF & NRoFF & NRoFF & $\mathrm{NRoFF}$ \\
\hline 146 & YUNSA & RoFF & RoFF & RoFF & RoFF & RoFF & NRoFF & RoFF & NRoFF \\
\hline \multirow{2}{*}{ TOTAL } & NO RISK OF FAILURE & 93 & 93 & 97 & 93 & 103 & 107 & 92 & 112 \\
\hline & RISK OF FINANCIAL FAILURE & 53 & 53 & 49 & 53 & 43 & 39 & 54 & 34 \\
\hline
\end{tabular}

Source: Authors' Compilation

When Table 9 is examined, a total of 112 companies showed success in the period of 2020/12, when the number of companies with the highest Taffler score was successful. It is seen that the Taffler score shows a total of 54 companies that failed in the 2019/12 period when the success was the lowest. Companies with financially successful Taffler score in all periods include: ACSEL, ATEKS, ALCAR, ALKA, ALKIM, AEFES, ARCLK, ARSAN, AYGAZ, BAKAB, BLCYT, BNTAS, BRSAN, BFREN, BUCIM, CCOLA, CEMTS, CMENT, CUSAN, DAGİ , DMSAS, DEVA, EGEEN, EGESER, EPLAS, ERBOS, EREGL, ERSU, FMZIP, FRIG, GEDZA, GENTS, HATEK, HEKTS, HURGZ, IHEVA, IHGZT, ISDMR, JANTS, KARTN, KENT, KERVTYA, KLMSN, RSTLKON , KUTPO, LUKSK, MAKTK, NUHCM, OTKAR, OYLUM, PARSN, PETKM, PETUN, PNSUT, PRZMA, RTLAB, SANFM, SEKUR, SELGD, SNPAM, TATGD, ULUSE, YATAS. Companies with unsuccessful Taffler scores in all periods include: ASUZU, BRMEN, CELHA, DGKLB, DYOBY, EKIZ, EMKEL, GUBRF, IZDMC, KAPLM, MRSHL, TIRE, SILVR, SKTAS, TUPRS, ULUUN, VESTL, VKING.

According to the formula in Table 3, the Zmijewski model's values of the BIST manufacturing companies within the scope of the research for the years 2019-2020 are given in Table 10.

Table 10: Zmijewski Values of BIST Manufacturing Companies for 2019-2020

\begin{tabular}{|c|c|c|c|c|c|c|c|c|c|}
\hline \multirow{2}{*}{ No. } & \multirow{2}{*}{ Code } & \multicolumn{8}{|c|}{ Zmijewski } \\
\hline & & $2019 / 3$ & $2020 / 3$ & $2019 / 6$ & $2020 / 6$ & $2019 / 9$ & $2020 / 9$ & $2019 / 12$ & $2020 / 12$ \\
\hline 1 & ACSEL & $-3,49$ & $-3,56$ & $-4,07$ & $-3,78$ & $-4,14$ & $-4,00$ & $-4,29$ & $-4,20$ \\
\hline 2 & ADEL & $-0,94$ & $-0,42$ & $-0,46$ & $-0,29$ & $-0,44$ & $-0,03$ & $-0,81$ & 0,10 \\
\hline 3 & AFYON & $-0,34$ & 0,11 & $-0,11$ & $-2,53$ & 0,10 & $-2,68$ & 0,33 & $-2,68$ \\
\hline 4 & AKCNS & $-1,21$ & $-1,08$ & $-1,34$ & $-1,30$ & $-1,55$ & $-1,61$ & $-1,72$ & $-1,64$ \\
\hline 5 & ATEKS & $-2,57$ & $-2,30$ & $-2,57$ & $-2,33$ & $-2,42$ & $-2,19$ & $-2,36$ & $-2,71$ \\
\hline 6 & AKSA & $-0,73$ & $-0,80$ & $-0,73$ & $-0,47$ & $-0,95$ & $-0,63$ & $-0,98$ & $-1,15$ \\
\hline 7 & ALCAR & $-2,62$ & $-2,30$ & $-2,27$ & $-2,18$ & $-2,66$ & $-2,34$ & $-2,63$ & $-2,55$ \\
\hline 8 & ALKA & $-1,26$ & $-2,28$ & $-2,27$ & $-3,47$ & $-2,46$ & $-3,80$ & $-3,29$ & $-3,98$ \\
\hline 9 & ALKIM & $-2,58$ & $-3,00$ & $-3,00$ & $-2,91$ & $-3,30$ & $-3,24$ & $-4,03$ & $-3,88$ \\
\hline 10 & AEFES & $-1,55$ & $-1,41$ & $-1,48$ & $-1,46$ & $-1,60$ & $-1,43$ & $-1,70$ & $-1,49$ \\
\hline 11 & ARCLK & $-0,19$ & $-0,19$ & $-0,20$ & $-0,32$ & $-0,26$ & $-0,38$ & $-0,31$ & $\begin{array}{l}-0,58 \\
\end{array}$ \\
\hline 12 & ARSAN & $-2,19$ & $-2,41$ & $-2,62$ & $-2,58$ & $-2,83$ & $-2,98$ & $-2,50$ & $-3,62$ \\
\hline 13 & ASUZU & $-0,39$ & $-0,35$ & $-0,32$ & $-0,28$ & $-0,49$ & $-0,17$ & $-0,69$ & $-0,31$ \\
\hline 14 & AVOD & $-0,95$ & $-0,44$ & $-1,07$ & $-0,34$ & $-0,92$ & $-0,03$ & $-0,76$ & $-0,21$ \\
\hline 15 & AYGAZ & $-1,00$ & $-0,99$ & $-1,36$ & $-0,72$ & $-1,47$ & $-0,67$ & $-1,70$ & $-1,02$ \\
\hline 16 & BAGFS & $-0,08$ & 0,08 & $-0,13$ & 0,27 & $-0,58$ & 0,73 & $-0,35$ & 0,34 \\
\hline 17 & BAKAB & $-0,95$ & $-0,95$ & $-0,84$ & $-0,76$ & $-1,09$ & $-0,86$ & $-1,11$ & $-1,13$ \\
\hline 18 & BANVT & $-1,08$ & $-1,22$ & $-1,57$ & $-1,03$ & $-1,62$ & $-0,93$ & $-1,89$ & $-0,39$ \\
\hline 19 & BRKSN & $-0,98$ & $-0,76$ & $-0,81$ & $-0,72$ & $-0,72$ & $-1,01$ & $-0,69$ & $-1,34$ \\
\hline 20 & BLCYT & $-2,27$ & $-3,71$ & $-2,61$ & $-3,57$ & $-3,37$ & $-3,92$ & $-4,25$ & $-3,97$ \\
\hline 21 & BNTAS & $-3,50$ & $-3,63$ & $-3,61$ & $-3,65$ & $-3,69$ & $-3,72$ & $-3,95$ & $-3,84$ \\
\hline 22 & BSOKE & $-0,17$ & 0,94 & 0,14 & 1,51 & 0,12 & 2,89 & 0,85 & 2,83 \\
\hline 23 & BTCIM & $-0,57$ & 0,13 & $-0,43$ & 0,49 & $-0,46$ & 1,33 & $-0,01$ & 1,26 \\
\hline 24 & BRKO & $-2,13$ & $-1,99$ & $-2,18$ & $-1,80$ & $-1,87$ & $-1,96$ & $-1,67$ & $-2,32$ \\
\hline
\end{tabular}

PAGE 103| Journal of Corporate Governance, Insurance, and Risk Management | 2021, VOL. 8, Series. 2 


\begin{tabular}{|c|c|c|c|c|}
\hline 25 & BRMEN & $-0,50$ & 1,56 & $-0,11$ \\
\hline 26 & BRSAN & $-1,05$ & $-1,06$ & $-1,23$ \\
\hline 27 & BOSSA & 0,34 & 0,08 & 0,28 \\
\hline 28 & BFREN & $-2,94$ & $-2,73$ & $-2,89$ \\
\hline 29 & BRİSA & 0,35 & 0,39 & 0,42 \\
\hline 30 & BURCE & $-0,59$ & $-1,37$ & $-0,56$ \\
\hline 31 & BURVA & $-0,30$ & $-0,16$ & $-0,34$ \\
\hline 32 & BUCiM & $-3,03$ & $-3,00$ & $-3,12$ \\
\hline 33 & CCOLA & 1,44 & 1,41 & 1,32 \\
\hline 34 & CELHA & 0,41 & 0,82 & 0,49 \\
\hline 35 & CEMAS & $-0,97$ & $-3,02$ & $-1,18$ \\
\hline 36 & CEMTS & $-3,26$ & $-3,46$ & $-3,76$ \\
\hline 37 & CMBTN & $-1,31$ & 0,00 & $-1,07$ \\
\hline 38 & CMENT & $-2,81$ & $-2,27$ & $-2,64$ \\
\hline 39 & CIMSA & $\begin{array}{ll}-0,88 \\
\end{array}$ & $-0,45$ & $-0,82$ \\
\hline 40 & CUSAN & $-1,25$ & $-1,66$ & $-1,48$ \\
\hline 41 & DAGI & $-1,20$ & $-1,07$ & $-1,52$ \\
\hline 42 & DARDL & 18,74 & 4,92 & 18,98 \\
\hline 43 & DMSAS & $-0,93$ & $-0,71$ & $-0,85$ \\
\hline 44 & DERIMM & 0,58 & 0,13 & 0,75 \\
\hline 45 & DESA & 0,15 & 0,03 & 0,09 \\
\hline 46 & DEVA & $-1,35$ & $-1,72$ & $-1,60$ \\
\hline 47 & DIRIT & 0,42 & 0,21 & $-0,73$ \\
\hline 48 & DİTAS & $-0,33$ & 0,06 & $-0,48$ \\
\hline 49 & DOBUR & $-0,80$ & $-0,56$ & $-0,83$ \\
\hline 50 & DGKLB & 1,08 & 1,51 & 1,25 \\
\hline 51 & DOGUB & $\begin{array}{c}-0,51 \\
\end{array}$ & $-2,66$ & $-2,82$ \\
\hline 52 & DOKTA & 0,71 & 1,13 & 0,51 \\
\hline 53 & DURDO & $-0,06$ & 0,30 & $-0,12$ \\
\hline 54 & DYOBY & 0,83 & 0,80 & 0,72 \\
\hline 55 & EGEEN & $-3,32$ & $-3,08$ & $-3,46$ \\
\hline 56 & EGGUB & $-1,55$ & $-0,79$ & $-1,70$ \\
\hline 57 & EGPRO & $-0,37$ & $-0,58$ & $-0,46$ \\
\hline 58 & EGESER & $-2,79$ & $-2,20$ & $-2,61$ \\
\hline 59 & EPLAS & $-0,62$ & $-1,93$ & $-0,77$ \\
\hline 60 & EKIZZ & 0,89 & 0,33 & 1,19 \\
\hline 61 & EMKEL & 0,67 & 0,18 & 0,33 \\
\hline 62 & EMNIS & 3,25 & 5,09 & 3,46 \\
\hline 63 & ERBOS & $-2,57$ & $-2,38$ & $-2,55$ \\
\hline 64 & EREGL & $-2,15$ & $-2,46$ & $-2,69$ \\
\hline 65 & ERSU & $-2,85$ & $-2,95$ & $-2,62$ \\
\hline 66 & FMZIP & $-4,34$ & $-4,31$ & $-5,08$ \\
\hline 67 & FROTO & $-0,05$ & $-0,13$ & $-0,32$ \\
\hline 68 & FORMT & $-1,32$ & $-0,48$ & $-1,23$ \\
\hline 69 & FRİGO & $-0,56$ & $-0,82$ & $-1,14$ \\
\hline 70 & GEDZA & $-2,76$ & $-2,72$ & $-2,76$ \\
\hline 71 & GENTS & $-2,68$ & $-2,82$ & $-2,58$ \\
\hline 72 & GEREL & $-1,62$ & $-0,83$ & $-1,03$ \\
\hline 73 & GOODY & $-0,59$ & $-1,01$ & $-0,78$ \\
\hline 74 & GOLTS & $\begin{array}{l}-0,73 \\
\end{array}$ & $-0,70$ & $-0,74$ \\
\hline 75 & GUBRF & 0,00 & 0,20 & 0,14 \\
\hline 76 & HATEK & $-2,38$ & $-2,61$ & $-1,96$ \\
\hline 77 & HEKTS & $-0,70$ & $-1,32$ & $-0,82$ \\
\hline 78 & HURGZ & $-2,70$ & $-2,59$ & $-2,58$ \\
\hline 79 & IHEVA & $-2,77$ & $-3,20$ & $-2,65$ \\
\hline 80 & IHGZT & $-3,22$ & $-3,06$ & $-3,07$ \\
\hline 81 & ISDMR & $-2,13$ & $-2,91$ & $-3,15$ \\
\hline 82 & IZDMC & 1,49 & 1,49 & 1,09 \\
\hline 83 & JANTS & $-2,25$ & $-1,92$ & $-2,70$ \\
\hline 84 & KAPLM & $\begin{array}{l}-0,49 \\
\end{array}$ & $-0,52$ & $-0,35$ \\
\hline 85 & KARDMD & $-1,28$ & $-0,83$ & $-1,39$ \\
\hline 86 & KARSN & 0,77 & $-0,04$ & 0,63 \\
\hline 87 & KRTEK & 0,30 & 0,56 & 0,41 \\
\hline 88 & KARTN & $\begin{array}{l}3,56 \\
\end{array}$ & $-3,41$ & $-3,70$ \\
\hline 89 & $\begin{array}{l}\text { KATMR } \\
\end{array}$ & 0,94 & 0,90 & 0,93 \\
\hline 90 & KENT & $-1,50$ & $-1,48$ & $-1,43$ \\
\hline 91 & KERVT & $\begin{array}{l}-0,13 \\
\end{array}$ & $\begin{array}{l}-0,69 \\
\end{array}$ & $-0,16$ \\
\hline 92 & KLMSN & $-0,08$ & 0,18 & $-0,49$ \\
\hline 93 & KONFRT & $-2,02$ & $-1,15$ & $-2,77$ \\
\hline 94 & KONYA & $-3,43$ & $-3,31$ & $-3,35$ \\
\hline 95 & KORDS & $-2,20$ & $-2,32$ & $-2,14$ \\
\hline 96 & KRSTL & $-2,89$ & $-2,67$ & $-2,60$ \\
\hline 97 & KUTPO & $-2,62$ & $-2,66$ & $-2,60$ \\
\hline 98 & LUKSK & $-1,57$ & $-1,49$ & $-1,58$ \\
\hline 99 & MAKTK & $-2,27$ & $-2,55$ & $-2,58$ \\
\hline 100 & MRSHL & 0,44 & 0,31 & 0,42 \\
\hline 101 & MNDRS & 0,55 & 1,27 & 0,76 \\
\hline 102 & OLMK & $-0,40$ & $-0,97$ & 0,13 \\
\hline 103 & TiRE & $-0,45$ & $-0,50$ & $-0,21$ \\
\hline 104 & NIBAS & $-2,88$ & $-3,26$ & $-2,44$ \\
\hline 105 & NUHCM & $-2,07$ & $-2,65$ & $-1,99$ \\
\hline 106 & OTKAR & 0,81 & 0,60 & 0,19 \\
\hline 107 & OYLUM & $\begin{array}{l}-1,08 \\
\end{array}$ & $-1,83$ & $-1,43$ \\
\hline 108 & OZBAL & 2,08 & $-0,06$ & 2,47 \\
\hline 109 & PARSN & $-0,73$ & $-1,11$ & $-0,83$ \\
\hline 110 & PENGD & 0,61 & $-1,11$ & 0,89 \\
\hline 111 & PETKM & $-0,45$ & $-0,41$ & $-0,49$ \\
\hline 112 & PETUN & $-2,65$ & $-2,85$ & $-2,96$ \\
\hline 113 & PINSU & 0,56 & 1,02 & 0,86 \\
\hline 114 & PNSUT & $-1,80$ & $-1,77$ & $-1,81$ \\
\hline 115 & PRZMA & $\begin{array}{l}-3,48 \\
\end{array}$ & \begin{tabular}{|l|}
$-3,72$ \\
\end{tabular} & $-3,36$ \\
\hline 116 & ROYAL & 1,83 & 1,30 & 1,91 \\
\hline 117 & RTLAB & \begin{tabular}{|l|}
$-3,93$ \\
\end{tabular} & $-3,36$ & $-4,16$ \\
\hline 118 & SANFM & 0,30 & 0,19 & 0,34 \\
\hline
\end{tabular}

PAGE 104| Journal of Corporate Governance, Insurance, and Risk Management | 2021, VOL. 8, Series. 2 


\begin{tabular}{|c|c|c|c|c|c|c|c|c|c|}
\hline 119 & SAMAT & 0,09 & 0,62 & 0,19 & $-0,37$ & 0,39 & $-0,39$ & 1,50 & $-0,78$ \\
\hline 120 & SARKY & $-0,48$ & $-0,20$ & $-0,33$ & $-0,21$ & $-0,42$ & $-0,30$ & $-0,31$ & $-0,54$ \\
\hline 121 & SAYAS & $-0,57$ & $-0,43$ & $-0,63$ & $-1,36$ & $-0,83$ & $-0,36$ & $-0,53$ & $-0,51$ \\
\hline 122 & SASA & $-1,10$ & $-0,42$ & $-1,09$ & $-0,40$ & $-1,28$ & 0,05 & $-1,11$ & $-0,24$ \\
\hline 123 & SEKUR & 0,01 & $-0,79$ & $\begin{array}{l}-0,73 \\
\end{array}$ & $-0,65$ & $-1,13$ & $-0,72$ & $-0,87$ & $-0,70$ \\
\hline 124 & SELGD & $-2,66$ & $-2,94$ & $-2,65$ & $-3,00$ & $-2,70$ & $-3,23$ & $-3,37$ & $-3,64$ \\
\hline 125 & SILVR & $-0,07$ & 0,32 & $-0,11$ & 0,37 & $-0,02$ & 0,48 & 0,33 & 0,21 \\
\hline 126 & SKTAS & 0,56 & 1,32 & 0,74 & 1,51 & $\begin{array}{l}0,78 \\
\end{array}$ & 0,77 & 0,93 & 0,39 \\
\hline 127 & SNPAM & $-2,27$ & $-2,75$ & $-2,98$ & $-2,64$ & $-3,13$ & $-2,42$ & $-3,44$ & $-2,85$ \\
\hline 128 & TATGD & $-2,00$ & $-1,78$ & $-1,75$ & $-1,74$ & $-1,69$ & $-1,43$ & $-2,00$ & $-2,24$ \\
\hline 129 & TMPOL & $-0,47$ & $-0,43$ & $-0,57$ & $-0,44$ & $-0,90$ & $-0,34$ & $-1,10$ & $-0,44$ \\
\hline 130 & TOASO & $-0,09$ & $-0,15$ & \begin{tabular}{|c|}
$-0,33$ \\
\end{tabular} & $-0,24$ & $-0,85$ & $-0,03$ & $-1,03$ & $-0,30$ \\
\hline 131 & TUCLK & $-0,82$ & $-0,62$ & $\begin{array}{l}-0,77 \\
\end{array}$ & $-0,47$ & $\begin{array}{l}-0,91 \\
\end{array}$ & $-0,39$ & $\begin{array}{l}-0,78 \\
\end{array}$ & $-0,52$ \\
\hline 132 & TUKAS & $\begin{array}{l}-0,39 \\
\end{array}$ & $-0,96$ & $\begin{array}{l}-0,70 \\
\end{array}$ & $-1,09$ & $-0,85$ & $-0,86$ & $-1,17$ & $-1,56$ \\
\hline 133 & TMSN & $-1,62$ & $-1,86$ & $-1,46$ & $-1,58$ & $-1,68$ & $-1,70$ & $-1,89$ & $-2,02$ \\
\hline 134 & TUPRS & 0,20 & 0,42 & 0,06 & 0,56 & 0,06 & 0,62 & $\begin{array}{l}0,03 \\
\end{array}$ & 0,52 \\
\hline 135 & PRKAB & $-0,69$ & $-0,91$ & $-0,65$ & $-1,04$ & $-0,97$ & $-0,86$ & $-1,15$ & $-0,93$ \\
\hline 136 & TTRAK & 0,30 & 0,09 & 0,27 & $-0,16$ & 0,02 & $-0,15$ & $-0,09$ & $-0,38$ \\
\hline 137 & TBORG & $-0,73$ & $-1,32$ & $-0,91$ & $-1,33$ & $-1,76$ & $-2,06$ & $-2,31$ & $-2,30$ \\
\hline 138 & ULUSE & $-3,83$ & $\begin{array}{l}-2,61 \\
\end{array}$ & $-3,65$ & $-2,74$ & $-3,68$ & $-2,87$ & $-3,67$ & $-2,11$ \\
\hline 139 & ULUUN & 0,15 & 0,22 & $-0,11$ & 0,14 & 0,04 & 0,19 & 0,11 & 0,00 \\
\hline 140 & USAK & $-0,60$ & $-0,29$ & $\begin{array}{l}-0,59 \\
\end{array}$ & $\begin{array}{l}-0,73 \\
\end{array}$ & $-0,46$ & $-1,02$ & $-0,40$ & $-1,04$ \\
\hline 141 & ULKER & $-0,74$ & $-0,66$ & $-0,86$ & $-0,90$ & $-0,97$ & $-0,91$ & $-1,11$ & $-0,89$ \\
\hline 142 & VESBE & $-0,91$ & $-0,79$ & $-0,72$ & $-1,17$ & $-1,05$ & $-1,04$ & $-1,17$ & $-1,65$ \\
\hline 143 & VESTL & 0,46 & 0,24 & 0,34 & 0,06 & 0,30 & 0,03 & 0,24 & $-0,44$ \\
\hline 144 & VKING & 1,05 & 1,54 & 1,42 & 1,72 & 1,74 & 2,15 & 1,87 & 2,25 \\
\hline 145 & YATAS & $-0,81$ & $-0,99$ & $-1,12$ & $-0,85$ & $-1,37$ & $-1,04$ & $-1,38$ & $-1,26$ \\
\hline 146 & YUNSA & 0,01 & $-0,21$ & $-0,06$ & $-0,22$ & 0,13 & $-0,45$ & $-0,19$ & $-0,46$ \\
\hline
\end{tabular}

Source: Authors' Compilation

The companies' determination within the research scope according to the Zmijewski values in Table 10 for the years 2019-2020 according to the threshold values (Table 3) is given in Table 11. RoFF (Risk of Financial Failure) was used for companies with a risk of financial failure and NRoFF (No Risk of Financial Failure) for companies with no risk of financial failure.

Table 11: Financial Status According to Zmijewski Model's Values of Manufacturing Companies for 20192020

\begin{tabular}{|c|c|c|c|c|c|c|c|c|c|}
\hline \multirow{2}{*}{ No. } & \multirow{2}{*}{ Code } & \multicolumn{8}{|c|}{ Zmijewski-Financial Status } \\
\hline & & $2019 / 3$ & $2020 / 3$ & $2019 / 6$ & $2020 / 6$ & $2019 / 9$ & $2020 / 9$ & 2019/12 & $2020 / 12$ \\
\hline 1 & ACSEL & NRoFF & NRoFF & NRoFF & NRoFF & NRoFF & NRoFF & NRoFF & $\mathrm{NRoFF}$ \\
\hline 2 & ADEL & NRoFF & NRoFF & NRoFF & NRoFF & NRoFF & NRoFF & NRoFF & RoFF \\
\hline 3 & AFYON & NRoFF & RoFF & NRoFF & NRoFF & RoFF & NRoFF & RoFF & NRoFF \\
\hline 4 & AKCNS & NRoFF & NRoFF & NRoFF & NRoFF & NRoFF & NRoFF & NRoFF & $\mathrm{NRoFF}$ \\
\hline 5 & ATEKS & NRoFF & NRoFF & NRoFF & NRoFF & NRoFF & NRoFF & NRoFF & $\mathrm{NRoFF}$ \\
\hline 6 & AKSA & NRoFF & NRoFF & NRoFF & NRoFF & NRoFF & NRoFF & NRoFF & NRoFF \\
\hline 7 & ALCAR & NRoFF & NRoFF & NRoFF & NRoFF & NRoFF & NRoFF & NRoFF & NRoFF \\
\hline 8 & ALKA & NRoFF & NRoFF & NRoFF & NRoFF & NRoFF & NRoFF & NRoFF & NRoFF \\
\hline 9 & ALKİM & NRoFF & NRoFF & NRoFF & NRoFF & NRoFF & NRoFF & NRoFF & NRoFF \\
\hline 10 & AEFES & NRoFF & NRoFF & NRoFF & NRoFF & NRoFF & NRoFF & NRoFF & NRoFF \\
\hline 11 & ARCLK & NRoFF & NRoFF & NRoFF & NRoFF & NRoFF & NRoFF & NRoFF & NRoFF \\
\hline 12 & ARSAN & NRoFF & NRoFF & NRoFF & NRoFF & NRoFF & NRoFF & NRoFF & NRoFF \\
\hline 13 & ASUZU & NRoFF & NRoFF & NRoFF & NRoFF & NRoFF & NRoFF & NRoFF & NRoFF \\
\hline 14 & AVOD & NRoFF & NRoFF & NRoFF & NRoFF & NRoFF & NRoFF & NRoFF & NRoFF \\
\hline 15 & AYGAZ & NRoFF & NRoFF & NRoFF & NRoFF & NRoFF & NRoFF & NRoFF & NRoFF \\
\hline 16 & BAGFS & NRoFF & RoFF & NRoFF & RoFF & NRoFF & RoFF & NRoFF & RoFF \\
\hline 17 & BAKAB & NRoFF & NRoFF & NRoFF & NRoFF & NRoFF & NRoFF & NRoFF & NRoFF \\
\hline 18 & BANVT & NRoFF & NRoFF & NRoFF & NRoFF & NRoFF & NRoFF & NRoFF & NRoFF \\
\hline 19 & BRKSN & NRoFF & NRoFF & NRoFF & NRoFF & NRoFF & NRoFF & NRoFF & NRoFF \\
\hline 20 & BLCYT & NRoFF & NRoFF & NRoFF & NRoFF & NRoFF & NRoFF & NRoFF & $\mathrm{NRoFF}$ \\
\hline 21 & BNTAS & NRoFF & NRoFF & NRoFF & NRoFF & NRoFF & NRoFF & NRoFF & NRoFF \\
\hline 22 & BSOKE & NRoFF & RoFF & RoFF & RoFF & RoFF & RoFF & RoFF & RoFF \\
\hline 23 & BTCIM & NRoFF & RoFF & NRoFF & RoFF & NRoFF & RoFF & NRoFF & RoFF \\
\hline 24 & BRKO & NRoFF & NRoFF & NRoFF & NRoFF & NRoFF & NRoFF & NRoFF & NRoFF \\
\hline 25 & BRMEN & NRoFF & RoFF & NRoFF & RoFF & RoFF & RoFF & RoFF & NRoFF \\
\hline 26 & BRSAN & NRoFF & NRoFF & NRoFF & NRoFF & NRoFF & NRoFF & NRoFF & NRoFF \\
\hline 27 & BOSSA & RoFF & RoFF & RoFF & RoFF & NRoFF & RoFF & NRoFF & NRoFF \\
\hline 28 & BFREN & NRoFF & NRoFF & NRoFF & NRoFF & NRoFF & NRoFF & NRoFF & NRoFF \\
\hline 29 & BRÍSA & RoFF & RoFF & RoFF & RoFF & RoFF & RoFF & RoFF & NRoFF \\
\hline 30 & BURCE & NRoFF & NRoFF & NRoFF & NRoFF & NRoFF & NRoFF & NRoFF & NRoFF \\
\hline 31 & BURVA & NRoFF & NRoFF & NRoFF & NRoFF & NRoFF & NRoFF & NRoFF & NRoFF \\
\hline 32 & BUCIM & NRoFF & NRoFF & NRoFF & NRoFF & NRoFF & NRoFF & NRoFF & NRoFF \\
\hline 33 & CCOLA & RoFF & RoFF & RoFF & RoFF & RoFF & RoFF & RoFF & RoFF \\
\hline 34 & CELHA & RoFF & RoFF & RoFF & RoFF & RoFF & RoFF & RoFF & RoFF \\
\hline 35 & CEMAS & NRoFF & NRoFF & NRoFF & NRoFF & NRoFF & NRoFF & NRoFF & NRoFF \\
\hline 36 & CEMTS & NRoFF & NRoFF & NRoFF & NRoFF & NRoFF & NRoFF & NRoFF & NRoFF \\
\hline 37 & CMBTN & NRoFF & RoFF & NRoFF & RoFF & NRoFF & RoFF & NRoFF & RoFF \\
\hline 38 & CMENT & NRoFF & NRoFF & NRoFF & NRoFF & NRoFF & NRoFF & NRoFF & NRoFF \\
\hline 39 & CIMSA & NRoFF & NRoFF & NRoFF & NRoFF & NRoFF & NRoFF & NRoFF & NRoFF \\
\hline 40 & CUSAN & NRoFF & NRoFF & NRoFF & NRoFF & NRoFF & NRoFF & NRoFF & NRoFF \\
\hline 41 & DAGİ & NRoFF & NRoFF & NRoFF & NRoFF & NRoFF & NRoFF & NRoFF & NRoFF \\
\hline 42 & DARDL & RoFF & RoFF & RoFF & RoFF & RoFF & NRoFF & RoFF & NRoFF \\
\hline 43 & DMSAS & NRoFF & NRoFF & NRoFF & NRoFF & NRoFF & NRoFF & NRoFF & NRoFF \\
\hline 44 & DERIM & RoFF & RoFF & RoFF & RoFF & RoFF & RoFF & RoFF & RoFF \\
\hline 45 & DESA & RoFF & RoFF & RoFF & RoFF & NRoFF & NRoFF & NRoFF & NRoFF \\
\hline 46 & DEVA & NRoFF & NRoFF & NRoFF & NRoFF & NRoFF & NRoFF & NRoFF & NRoFF \\
\hline
\end{tabular}

PAGE 105| Journal of Corporate Governance, Insurance, and Risk Management | 2021, VOL. 8, Series. 2 


\begin{tabular}{|c|c|c|c|c|}
\hline 47 & DIRIT & RoFF & RoFF & NRoFF \\
\hline 48 & DİTAS & NRoFF & RoFF & NRoFF \\
\hline 49 & DOBUR & NRoFF & NRoFF & NRoFF \\
\hline 50 & DGKLB & RoFF & RoFF & RoFF \\
\hline 51 & DOGUB & NRoFF & NRoFF & NRoFF \\
\hline 52 & DOKTA & RoFF & RoFF & RoFF \\
\hline 53 & DURDO & NRoFF & RoFF & NRoFF \\
\hline 54 & DYOBY & RoFF & RoFF & RoFF \\
\hline 55 & EGEEN & NRoFF & NRoFF & NRoFF \\
\hline 56 & EGGUB & NRoFF & NRoFF & NRoFF \\
\hline 57 & EGPRO & NRoFF & NRoFF & NRoFF \\
\hline 58 & $\begin{array}{l}\text { EGESER } \\
\end{array}$ & NRoFF & NRoFF & NRoFF \\
\hline 59 & EPLAS & NRoFF & NRoFF & NRoFF \\
\hline 60 & EKİZ & RoFF & RoFF & RoFF \\
\hline 61 & EMKEL & RoFF & RoFF & RoFF \\
\hline 62 & EMNIS & RoFF & RoFF & RoFF \\
\hline 63 & ERBOS & NRoFF & NRoFF & NRoFF \\
\hline 64 & EREGL & NRoFF & NRoFF & NRoFF \\
\hline 65 & ERSU & NRoFF & NRoFF & NRoFF \\
\hline 66 & FMZIP & NRoFF & NRoFF & NRoFF \\
\hline 67 & FROTO & NRoFF & NRoFF & NRoFF \\
\hline 68 & FORMT & NRoFF & NRoFF & NRoFF \\
\hline 69 & FRİGO & NRoFF & NRoFF & NRoFF \\
\hline 70 & GEDZA & NRoFF & NRoFF & NRoFF \\
\hline 71 & GENTS & NRoFF & NRoFF & NRoFF \\
\hline 72 & GEREL & NRoFF & NRoFF & NRoFF \\
\hline 73 & GOODY & NRoFF & NRoFF & NRoFF \\
\hline 74 & GOLTS & NRoFF & NRoFF & NRoFF \\
\hline 75 & GUBRF & RoFF & RoFF & RoFF \\
\hline 76 & HATEK & NRoFF & NRoFF & NRoFF \\
\hline 77 & HEKTS & NRoFF & NRoFF & NRoFF \\
\hline 78 & HURGZ & NRoFF & NRoFF & NRoFF \\
\hline 79 & IHEVA & NRoFF & NRoFF & NRoFF \\
\hline 80 & IHGZT & NRoFF & NRoFF & NRoFF \\
\hline 81 & ISDMR & NRoFF & NRoFF & NRoFF \\
\hline 82 & IZDMC & RoFF & RoFF & RoFF \\
\hline 83 & JANTS & NRoFF & NRoFF & NRoFF \\
\hline 84 & KAPLM & NRoFF & NRoFF & NRoFF \\
\hline 85 & KARDMD & NRoFF & NRoFF & NRoFF \\
\hline 86 & KARSN & RoFF & NRoFF & RoFF \\
\hline 87 & KRTEK & RoFF & RoFF & RoFF \\
\hline 88 & KARTN & NRoFF & NRoFF & NRoFF \\
\hline 89 & KATMR & RoFF & RoFF & RoFF \\
\hline 90 & KENT & NRoFF & NRoFF & NRoFF \\
\hline 91 & KERVT & NRoFF & NRoFF & NRoFF \\
\hline 92 & KLMSN & NRoFF & RoFF & NRoFF \\
\hline 93 & KONFRT & NRoFF & NRoFF & NRoFF \\
\hline 94 & KONYA & NRoFF & NRoFF & NRoFF \\
\hline 95 & KORDS & NRoFF & NRoFF & NRoFF \\
\hline 96 & KRSTL & NRoFF & NRoFF & NRoFF \\
\hline 97 & KUTPO & NRoFF & NRoFF & NRoFF \\
\hline 98 & LUKSK & NRoFF & NRoFF & NRoFF \\
\hline 99 & MAKTK & NRoFF & NRoFF & NRoFF \\
\hline 100 & MRSHL & RoFF & RoFF & RoFF \\
\hline 101 & MNDRS & RoFF & RoFF & RoFF \\
\hline 102 & OLMK & NRoFF & NRoFF & RoFF \\
\hline 103 & TIRE & NRoFF & NRoFF & NRoFF \\
\hline 104 & NIBAS & NRoFF & NRoFF & NRoFF \\
\hline 105 & NUHCM & NRoFF & NRoFF & NRoFF \\
\hline 106 & OTKAR & RoFF & RoFF & RoFF \\
\hline 107 & OYLUM & NRoFF & NRoFF & NRoFF \\
\hline 108 & OZBAL & RoFF & NRoFF & RoFF \\
\hline 109 & PARSN & NRoFF & NRoFF & NRoFF \\
\hline 110 & PENGD & RoFF & NRoFF & RoFF \\
\hline 111 & PETKM & NRoFF & NRoFF & NRoFF \\
\hline 112 & PETUN & NRoFF & NRoFF & NRoFF \\
\hline 113 & PINSU & RoFF & RoFF & RoFF \\
\hline 114 & PNSUT & NRoFF & NRoFF & NRoFF \\
\hline 115 & PRZMA & NRoFF & NRoFF & NRoFF \\
\hline 116 & ROYAL & RoFF & RoFF & RoFF \\
\hline 117 & RTLAB & NRoFF & NRoFF & NRoFF \\
\hline 118 & SANFM & RoFF & RoFF & RoFF \\
\hline 119 & SAMAT & RoFF & RoFF & RoFF \\
\hline 120 & SARKY & NRoFF & NRoFF & NRoFF \\
\hline 121 & SAYAS & NRoFF & NRoFF & NRoFF \\
\hline 122 & SASA & NRoFF & NRoFF & NRoFF \\
\hline 123 & SEKUR & RoFF & NRoFF & NRoFF \\
\hline 124 & SELGD & NRoFF & NRoFF & NRoFF \\
\hline 125 & SILVR & NRoFF & RoFF & NRoFF \\
\hline 126 & SKTAS & RoFF & RoFF & RoFF \\
\hline 127 & SNPAM & NRoFF & NRoFF & NRoFF \\
\hline 128 & TATGD & NRoFF & NRoFF & NRoFF \\
\hline 129 & TMPOL & NRoFF & NRoFF & NRoFF \\
\hline 130 & TOASO & NRoFF & NRoFF & NRoFF \\
\hline 131 & TUCLK & NRoFF & NRoFF & NRoFF \\
\hline 132 & TUKAS & NRoFF & NRoFF & NRoFF \\
\hline 133 & TMSN & NRoFF & NRoFF & NRoFF \\
\hline 134 & TUPRS & RoFF & RoFF & RoFF \\
\hline 135 & PRKAB & NRoFF & NRoFF & NRoFF \\
\hline 136 & TTRAK & RoFF & RoFF & RoFF \\
\hline 137 & TBORG & NRoFF & NRoFF & NRoFF \\
\hline 138 & ULUSE & NRoFF & NRoFF & NRoFF \\
\hline 139 & ULUUN & RoFF & RoFF & NRoFF \\
\hline 140 & USAK & NRoFF & NRoFF & NRoFF \\
\hline
\end{tabular}

PAGE 106| Journal of Corporate Governance, Insurance, and Risk Management | 2021, VOL. 8, Series. 2 


\begin{tabular}{|c|l|c|c|c|c|c|c|c|c|}
\hline 141 & ULKER & NRoFF & NRoFF & NRoFF & NRoFF & NRoFF & NRoFF & NRoFF & NRoFF \\
\hline 142 & VESBE & NRoFF & NRoFF & NRoFF & NRoFF & NRoFF & NRoFF & NRoFF & NRoFF \\
\hline 143 & VESTL & RoFF & RoFF & RoFF & RoFF & RoFF & RoFF & RoFF & NRoFF \\
\hline 144 & VKING & RoFF & RoFF & RoFF & RoFF & RoFF & RoFF & RoFF & RoFF \\
\hline 145 & YATAS & NRoFF & NRoFF & NRoFF & NRoFF & NRoFF & NRoFF & NRoFF & NRoFF \\
\hline 146 & YUNSA & RoFF & NRoFF & NRoFF & NRoFF & NRoFF & NRoFF & NRoFF & NRoFF \\
\hline \multirow{2}{*}{ TOTAL } & NO RISK OF FAILURE & $\mathbf{1 1 0}$ & $\mathbf{1 0 5}$ & $\mathbf{1 1 2}$ & $\mathbf{1 0 8}$ & $\mathbf{1 1 1}$ & $\mathbf{1 1 0}$ & $\mathbf{1 1 6}$ & $\mathbf{1 2 3}$ \\
\cline { 2 - 17 } & RISK OF FINANCIAL FAILURE & $\mathbf{3 6}$ & $\mathbf{4 1}$ & $\mathbf{3 4}$ & $\mathbf{3 8}$ & $\mathbf{3 5}$ & $\mathbf{3 6}$ & $\mathbf{3 0}$ & $\mathbf{2 3}$ \\
\hline
\end{tabular}

Source: Authors' Compilation

According to Table 11, the period with the highest number of successful companies with a Zmijewski score is 2020/12 with a total of 123 companies. The period with the highest number of unsuccessful companies is 2020/3. The manufacturing companies that failed in all periods are: CCOLA, CELHA, DERIM, DGKLB, DOKTA, IZDMC, KATMR, MNDRS, PINSU, ROYAL, SKTAS, TUPRS, VKING.

Those with successful financial scores in all periods include: ACSEL, AKCNS, ATEKS, AKSA, ALCAR, ALKA, ALKIM, AEFES, ARCLK, ARSAN, ASUZU, AVOD, AYGAZ, BAKAB, BANVT, BRKSN, BLCYT, BNTAS, BRKO, BRSAN, BFREN, BURCE , BURVA, BUCIM, CEMAS, CEMTS, CMENT, CIMSA, CUSAN, DAGI, DMSAS, DEVA, DOBUR, DOGUB, EGEEN, EGGUB, EGPRO, EGESER, EPLAS, ERBOS, EREGL, ERSU, FMZIP, FROTO, FORMT, FRIG, GED , GENTS, GEREL, GOODY, GOLTS, HATEK, HEKTS, HURGZ, IHEVA, IHGZT, ISDMR, JANTS, KAPLM, KARDMD, KARTN, KENT, KERVT, KONFRT, KONYA, KORDS, KRSTL, KUTPO, LUKSK, MAKTK, TIRE, NIBAS, NUHCM, OYLUM, PARSN, PETKM, YATAS PETUN, PNSUT, PRZMA, RTLAB, SARKY, SAYDAS, SARKY, SAYDAS , TATGD, TMPOL, TOASO, TUCLK, TUKAS, TMSN, PRKAB, TBORG, ULUSE, USAK, ULKER, VESBE.

Comparative information on the financial status of the BIST manufacturing companies for the periods 2019/12 and 2020/12 for financial failure models within the scope of the research is given in Table 12.

Table 12: Evaluation of Financial Failure Models Used in the Research for the Periods of 2019/12 and 2020/12

\begin{tabular}{|c|c|c|c|c|c|c|c|c|c|}
\hline \multirow{2}{*}{ No. } & \multirow{2}{*}{ Code } & \multicolumn{4}{|c|}{$2019 / 12$} & \multicolumn{4}{|c|}{$2020 / 12$} \\
\hline & & Altman & Springate & Taffler & Zmijewski & Altman & Springate & Taffler & Zmijewski \\
\hline 1 & ACSEL & NRoFF & NRoFF & NRoFF & NRoFF & NRoFF & NRoFF & NRoFF & NRoFF \\
\hline 2 & \begin{tabular}{|l|} 
ADEL \\
\end{tabular} & RoFF & RoFF & NRoFF & NRoFF & RoFF & RoFF & RoFF & RoFF \\
\hline 3 & AFYON & RoFF & RoFF & RoFF & RoFF & NRoFF & RoFF & RoFF & NRoFF \\
\hline 4 & AKCNS & RoFF & RoFF & NRoFF & NRoFF & UN & RoFF & NRoFF & NRoFF \\
\hline 5 & ATEKS & RoFF & RoFF & NRoFF & NRoFF & UN & RoFF & NRoFF & NRoFF \\
\hline 6 & \begin{tabular}{|l|} 
AKSA \\
\end{tabular} & RoFF & RoFF & NRoFF & NRoFF & RoFF & RoFF & NRoFF & NRoFF \\
\hline 7 & ALCAR & NRoFF & NRoFF & NRoFF & NRoFF & NRoFF & NRoFF & NRoFF & NRoFF \\
\hline 8 & \begin{tabular}{|l|} 
ALKA \\
\end{tabular} & NRoFF & NRoFF & NRoFF & NRoFF & NRoFF & NRoFF & NRoFF & NRoFF \\
\hline 9 & ALKIM & UN & NRoFF & NRoFF & NRoFF & NRoFF & NRoFF & NRoFF & NRoFF \\
\hline 10 & AEFES & RoFF & RoFF & NRoFF & NRoFF & RoFF & RoFF & NRoFF & NRoFF \\
\hline 11 & ARCLK & RoFF & RoFF & NRoFF & NRoFF & RoFF & NRoFF & NRoFF & NRoFF \\
\hline 12 & \begin{tabular}{|l|} 
ARSAN \\
\end{tabular} & RoFF & RoFF & NRoFF & NRoFF & UN & RoFF & NRoFF & NRoFF \\
\hline 13 & ASUZU & RoFF & RoFF & RoFF & NRoFF & RoFF & RoFF & RoFF & NRoFF \\
\hline 14 & AVOD & RoFF & RoFF & RoFF & NRoFF & UN & RoFF & RoFF & NRoFF \\
\hline 15 & AYGAZ & UN & RoFF & NRoFF & NRoFF & RoFF & RoFF & NRoFF & NRoFF \\
\hline 16 & BAGFS & RoFF & RoFF & RoFF & NRoFF & RoFF & RoFF & NRoFF & RoFF \\
\hline 17 & BAKAB & RoFF & RoFF & NRoFF & NRoFF & UN & RoFF & NRoFF & NRoFF \\
\hline 18 & BANVT & UN & RoFF & NRoFF & NRoFF & UN & RoFF & RoFF & NRoFF \\
\hline 19 & BRKSN & RoFF & RoFF & NRoFF & NRoFF & UN & NRoFF & NRoFF & NRoFF \\
\hline 20 & BLCYT & UN & NRoFF & NRoFF & NRoFF & NRoFF & RoFF & NRoFF & NRoFF \\
\hline 21 & BNTAS & NRoFF & NRoFF & NRoFF & NRoFF & NRoFF & RoFF & NRoFF & NRoFF \\
\hline 22 & BSOKE & RoFF & RoFF & RoFF & RoFF & RoFF & RoFF & RoFF & RoFF \\
\hline 23 & BTCIM & RoFF & RoFF & RoFF & NRoFF & RoFF & RoFF & RoFF & RoFF \\
\hline 24 & \begin{tabular}{|l|} 
BRKO \\
\end{tabular} & RoFF & RoFF & RoFF & NRoFF & RoFF & RoFF & NRoFF & NRoFF \\
\hline 25 & BRMEN & RoFF & RoFF & RoFF & RoFF & RoFF & RoFF & RoFF & NRoFF \\
\hline 26 & BRSAN & RoFF & RoFF & NRoFF & NRoFF & RoFF & RoFF & NRoFF & NRoFF \\
\hline 27 & BOSSA & RoFF & RoFF & NRoFF & NRoFF & RoFF & RoFF & RoFF & NRoFF \\
\hline 28 & BFREN & NRoFF & NRoFF & NRoFF & NRoFF & NRoFF & NRoFF & NRoFF & NRoFF \\
\hline 29 & \begin{tabular}{|l|} 
BRİSA \\
\end{tabular} & RoFF & RoFF & RoFF & RoFF & RoFF & RoFF & NRoFF & NRoFF \\
\hline 30 & BURCE & RoFF & RoFF & NRoFF & NRoFF & RoFF & RoFF & NRoFF & NRoFF \\
\hline 31 & BURVA & NRoFF & NRoFF & NRoFF & RoFF & NRoFF & RoFF & NRoFF & NRoFF \\
\hline 32 & \begin{tabular}{|l|} 
BUCIM \\
\end{tabular} & UN & RoFF & NRoFF & NRoFF & NRoFF & NRoFF & NRoFF & NRoFF \\
\hline 33 & CCOLA & RoFF & RoFF & NRoFF & RoFF & RoFF & RoFF & NRoFF & RoFF \\
\hline 34 & CELHA & RoFF & RoFF & RoFF & RoFF & RoFF & RoFF & RoFF & RoFF \\
\hline 35 & CEMAS & RoFF & RoFF & RoFF & NRoFF & NRoFF & RoFF & NRoFF & NRoFF \\
\hline
\end{tabular}

PAGE 107| Journal of Corporate Governance, Insurance, and Risk Management | 2021, VOL. 8, Series. 2 


\begin{tabular}{|c|c|c|c|c|}
\hline 36 & CEMTS & NRoFF & NRoFF & RoFF \\
\hline 37 & CMBTN & RoFF & RoFF & RoFF \\
\hline 38 & CMENT & UN & RoFF & NRoFF \\
\hline 39 & CIMSA & RoFF & RoFF & RoFF \\
\hline 40 & CUSAN & RoFF & RoFF & NRoFF \\
\hline 41 & \begin{tabular}{|l|} 
DAGİ \\
\end{tabular} & RoFF & RoFF & NRoFF \\
\hline 42 & DARDL & RoFF & RoFF & RoFF \\
\hline 43 & DMSAS & RoFF & RoFF & NRoFF \\
\hline 44 & DERIM & RoFF & RoFF & RoFF \\
\hline 45 & DESA & RoFF & NRoFF & RoFF \\
\hline 46 & \begin{tabular}{|l|} 
DEVA \\
\end{tabular} & UN & RoFF & NRoFF \\
\hline 47 & \begin{tabular}{|l|} 
DIRIT \\
\end{tabular} & RoFF & RoFF & RoFF \\
\hline 48 & \begin{tabular}{|l|} 
DİTAS \\
\end{tabular} & RoFF & RoFF & RoFF \\
\hline 49 & DOBUR & UN & NRoFF & NRoFF \\
\hline 50 & DGKLB & RoFF & RoFF & RoFF \\
\hline 51 & DOGUB & NRoFF & RoFF & NRoFF \\
\hline 52 & DOKTA & RoFF & RoFF & NRoFF \\
\hline 53 & DURDO & RoFF & RoFF & NRoFF \\
\hline 54 & DYOBY & RoFF & RoFF & RoFF \\
\hline 55 & EGEEN & NRoFF & NRoFF & NRoFF \\
\hline 56 & EGGUB & UN & RoFF & RoFF \\
\hline 57 & EGPRO & RoFF & RoFF & NRoFF \\
\hline 58 & \begin{tabular}{|l|} 
EGESER \\
\end{tabular} & UN & RoFF & NRoFF \\
\hline 59 & \begin{tabular}{|l|} 
EPLAS \\
\end{tabular} & UN & RoFF & NRoFF \\
\hline 60 & EKíz & RoFF & RoFF & RoFF \\
\hline 61 & EMKEL & RoFF & RoFF & RoFF \\
\hline 62 & \begin{tabular}{|l|} 
EMNIS \\
\end{tabular} & RoFF & RoFF & NRoFF \\
\hline 63 & \begin{tabular}{|l|} 
ERBOS \\
\end{tabular} & UN & RoFF & NRoFF \\
\hline 64 & \begin{tabular}{|l|} 
EREGL \\
\end{tabular} & RoFF & RoFF & NRoFF \\
\hline 65 & \begin{tabular}{|l|} 
ERSU \\
\end{tabular} & UN & RoFF & NRoFF \\
\hline 66 & \begin{tabular}{|l|} 
FMZIP \\
\end{tabular} & NRoFF & NRoFF & NRoFF \\
\hline 67 & FROTO & UN & RoFF & NRoFF \\
\hline 68 & FORMT & RoFF & RoFF & RoFF \\
\hline 69 & \begin{tabular}{|l|} 
FRİGO \\
\end{tabular} & RoFF & RoFF & NRoFF \\
\hline 70 & GEDZA & UN & RoFF & NRoFF \\
\hline 71 & GENTS & UN & RoFF & NRoFF \\
\hline 72 & GEREL & RoFF & RoFF & RoFF \\
\hline 73 & GOODY & $\mathrm{UN}$ & NRoFF & NRoFF \\
\hline 74 & GOLTS & RoFF & RoFF & NRoFF \\
\hline 75 & GUBRF & RoFF & RoFF & RoFF \\
\hline 76 & HATEK & RoFF & RoFF & NRoFF \\
\hline 77 & HEKTS & RoFF & RoFF & NRoFF \\
\hline 78 & HURGZ & RoFF & RoFF & NRoFF \\
\hline 79 & \begin{tabular}{|l|} 
IHEVA \\
\end{tabular} & NRoFF & NRoFF & NRoFF \\
\hline 80 & \begin{tabular}{|l|} 
IHGZT \\
\end{tabular} & NRoFF & NRoFF & NRoFF \\
\hline 81 & \begin{tabular}{|l|} 
ISDMR \\
\end{tabular} & UN & RoFF & NRoFF \\
\hline 82 & IZDMC & RoFF & RoFF & RoFF \\
\hline 83 & \begin{tabular}{|l|} 
JANTS \\
\end{tabular} & RoFF & RoFF & NRoFF \\
\hline 84 & KAPLM & RoFF & RoFF & RoFF \\
\hline 85 & \begin{tabular}{|l|} 
KARDMD \\
\end{tabular} & RoFF & RoFF & NRoFF \\
\hline 86 & KARSN & RoFF & RoFF & RoFF \\
\hline 87 & KRTEK & RoFF & RoFF & RoFF \\
\hline 88 & KARTN & RoFF & NRoFF & NRoFF \\
\hline 89 & KATMR & RoFF & RoFF & NRoFF \\
\hline 90 & KENT & NRoFF & RoFF & NRoFF \\
\hline 91 & KERVT & RoFF & RoFF & NRoFF \\
\hline 92 & KLMSN & RoFF & RoFF & NRoFF \\
\hline 93 & \begin{tabular}{|l|} 
KONFRT \\
\end{tabular} & RoFF & RoFF & RoFF \\
\hline 94 & KONYA & NRoFF & RoFF & NRoFF \\
\hline 95 & KORDS & RoFF & RoFF & NRoFF \\
\hline 96 & \begin{tabular}{|l|} 
KRSTLL \\
\end{tabular} & NRoFF & RoFF & NRoFF \\
\hline 97 & KUTPO & UN & NRoFF & NRoFF \\
\hline 98 & LUKSK & RoFF & RoFF & NRoFF \\
\hline 99 & MAKTK & NRoFF & RoFF & NRoFF \\
\hline 100 & MRSHL & UN & RoFF & RoFF \\
\hline 101 & \begin{tabular}{|l|} 
MNDRS \\
\end{tabular} & RoFF & RoFF & RoFF \\
\hline 102 & OLMK & RoFF & RoFF & RoFF \\
\hline 103 & TIRE & RoFF & RoFF & RoFF \\
\hline 104 & NIBAS & NRoFF & RoFF & NRoFF \\
\hline 105 & \begin{tabular}{|l|} 
NUHCM \\
\end{tabular} & UN & RoFF & NRoFF \\
\hline 106 & OTKAR & UN & NRoFF & NRoFF \\
\hline 107 & \begin{tabular}{|l|} 
OYLUM \\
\end{tabular} & RoFF & RoFF & NRoFF \\
\hline 108 & OZBAL & RoFF & RoFF & RoFF \\
\hline 109 & PARSN & RoFF & RoFF & NRoFF \\
\hline 110 & PENGD & RoFF & RoFF & RoFF \\
\hline 111 & \begin{tabular}{|l|} 
PETKM \\
\end{tabular} & RoFF & RoFF & NRoFF \\
\hline 112 & PETUN & UN & RoFF & NRoFF \\
\hline 113 & \begin{tabular}{|l|} 
PINSU \\
\end{tabular} & RoFF & RoFF & RoFF \\
\hline 114 & \begin{tabular}{|l|} 
PNSUT \\
\end{tabular} & RoFF & RoFF & NRoFF \\
\hline 115 & PRZMA & NRoFF & RoFF & NRoFF \\
\hline 116 & ROYAL & RoFF & RoFF & RoFF \\
\hline 117 & RTLAB & NRoFF & RoFF & NRoFF \\
\hline 118 & \begin{tabular}{|l|} 
SANFM \\
\end{tabular} & RoFF & RoFF & NRoFF \\
\hline 119 & \begin{tabular}{|l|} 
SAMAT \\
\end{tabular} & RoFF & RoFF & RoFF \\
\hline 120 & SARKY & RoFF & RoFF & RoFF \\
\hline 121 & SAYAS & RoFF & RoFF & RoFF \\
\hline 122 & \begin{tabular}{|l|} 
SASA \\
\end{tabular} & RoFF & RoFF & RoFF \\
\hline 123 & \begin{tabular}{|l|} 
SEKUR \\
\end{tabular} & RoFF & RoFF & NRoFF \\
\hline 124 & \begin{tabular}{|l|} 
SELGD \\
\end{tabular} & NRoFF & NRoFF & NRoFF \\
\hline 125 & \begin{tabular}{|l|} 
SILVR \\
\end{tabular} & RoFF & RoFF & RoFF \\
\hline 126 & \begin{tabular}{|l|} 
SKTAS \\
\end{tabular} & RoFF & RoFF & RoFF \\
\hline 127 & SNPAM & NRoFF & RoFF & NRoFF \\
\hline 128 & TATGD & UN & NRoFF & NRoFF \\
\hline 129 & TMPOL & RoFF & RoFF & NRoFF \\
\hline
\end{tabular}

PAGE 108| Journal of Corporate Governance, Insurance, and Risk Management | 2021, VOL. 8, Series. 2 


\begin{tabular}{|c|c|c|c|c|c|c|c|c|c|}
\hline 130 & TOASO & RoFF & RoFF & NRoFF & NRoFF & RoFF & RoFF & RoFF & NRoFF \\
\hline 131 & TUCLK & RoFF & RoFF & RoFF & NRoFF & RoFF & RoFF & NRoFF & NRoFF \\
\hline 132 & TUKAS & NRoFF & RoFF & NRoFF & NRoFF & UN & RoFF & NRoFF & NRoFF \\
\hline 133 & \begin{tabular}{|l|} 
TMSN \\
\end{tabular} & RoFF & RoFF & NRoFF & NRoFF & UN & RoFF & NRoFF & NRoFF \\
\hline 134 & \begin{tabular}{|l|l} 
TUPRS \\
\end{tabular} & RoFF & RoFF & RoFF & RoFF & RoFF & RoFF & RoFF & RoFF \\
\hline 135 & PRKAB & RoFF & RoFF & NRoFF & NRoFF & NRoFF & RoFF & NRoFF & NRoFF \\
\hline 136 & TTRAK & UN & RoFF & NRoFF & NRoFF & NRoFF & NRoFF & NRoFF & NRoFF \\
\hline 137 & TBORG & UN & NRoFF & NRoFF & NRoFF & NRoFF & NRoFF & NRoFF & NRoFF \\
\hline 138 & ULUSE & NRoFF & NRoFF & NRoFF & NRoFF & NRoFF & NRoFF & NRoFF & NRoFF \\
\hline 139 & ULUUN & RoFF & RoFF & RoFF & RoFF & RoFF & RoFF & RoFF & NRoFF \\
\hline 140 & \begin{tabular}{|l|} 
USAK \\
\end{tabular} & RoFF & RoFF & RoFF & NRoFF & RoFF & RoFF & NRoFF & NRoFF \\
\hline 141 & ULKER & RoFF & RoFF & NRoFF & NRoFF & RoFF & RoFF & NRoFF & NRoFF \\
\hline 142 & VESBE & RoFF & RoFF & RoFF & NRoFF & UN & RoFF & NRoFF & NRoFF \\
\hline 143 & \begin{tabular}{|l|} 
VESTL \\
\end{tabular} & RoFF & RoFF & RoFF & RoFF & RoFF & RoFF & RoFF & NRoFF \\
\hline 144 & VKING & RoFF & RoFF & RoFF & RoFF & RoFF & RoFF & RoFF & RoFF \\
\hline 145 & $\begin{array}{l}\text { YATAS } \\
\end{array}$ & UN & NRoFF & NRoFF & NRoFF & UN & NRoFF & NRoFF & NRoFF \\
\hline 146 & YUNSA & RoFF & RoFF & RoFF & NRoFF & RoFF & RoFF & NRoFF & $\mathrm{NRoFF}$ \\
\hline \multirow{3}{*}{ TOTAL } & NO RISK OF FAILURE & 23 & 24 & 92 & 114 & 50 & 37 & 110 & 124 \\
\hline & RISK OF FINANCIAL FAILURE & 96 & 122 & 54 & 32 & 65 & 109 & 36 & 22 \\
\hline & \begin{tabular}{|l|} 
UNCERTAIN \\
\end{tabular} & 27 & - & - & - & 31 & - & - & - \\
\hline
\end{tabular}

Source: Authors' Compilation

According to the data in Table 12, it is seen that the BIST manufacturing companies with successful Altman Z, Springate, Taffler, Zmijewski scores in the quarterly periods of 2019 and 2020 are: ACSEL, ALCAR, ALKA, BFREN, EGEEN, FMZIP, IHEVA, ULUSE. BIST companies with unsuccessful scores were: BSOKE, CELHA, DERIM, DIRIT, DGKLB, EMKEL, IZDMC, PINSU, SILVR, SKTAS, TUPRS, VKING.

\section{CONCLUSION}

This study aimed to analyze the financial failure risk of BIST manufacturing companies in the COVID-19 period by comparing 2019 and 2020 with four different financial failure models. Analysis was carried out with the most used Altman (1968), Springate (1978), Taffler (1983) and Zmijewski (1984) financial failure models in the literature, with quarterly data from 146 companies.

The analysis results show that while the risk of financial failure decreased in all four models, the number of financially successful companies increased when the 2019 and 2020 quarterly data were compared. This can be interpreted as meaning that the BIST manufacturing industry companies are less affected by the COVID-19 process when comparing 2019 and 2020. While the risk of financial failure decreased in the quarter of 2019 , the number of financially successful companies increased. This increase is also valid for the quarters of 2020. While the number of financially failed companies in the first quarter of 2019 and 2020 decreased in Altman and Zmijewski models, there was no change in the number of financially successful or unsuccessful companies in Springate and Tafller models. The difference between these models in the first quarter is not found in the following quarters. In the Zmijewski and Taffler models, financially successful companies by quarter are higher than in the Altman and Springate models.

On the other hand, in Altman and Springate models, the number of companies at risk of financial failure is considerably higher than in Zmijewski and Taffler models. This may be due to the fact that the variables in the models are similar to each other. According to the Springate model, the number of 
companies at risk of financial failure is higher than the number of companies calculated according to the Altman model. It can be explained by the Altman model using market value, total debt, and retained earnings as variables. As a result of the calculations made according to the four financial failure models, some companies are at risk of financial failure in all quarters. Companies at risk of financial failure in all quarters in both the Altman and Springate models are; ADEL, AKSA, AEFES, BAGFS, BTCIM, BRKO, BRMEN, BRSAN, BOSSA, BRISA, BURCE, CELHA, CIMSA, DAGI, DARDNL, DERIM, DIRIT, DGKLB, DOKTA, DURDO, DYOBY, EKIZ, EMKEL, IZDMC, KAR KARSN, KLMSN, KORDS, MNDRS, PINSU, PNSUT, ROYAL, SANFM, TOASO, TUCLK, TUPRS, ULUUN, USAK, VESTL, VKING, YUNSA. Companies at risk of financial failure in all quarters in the Altman, Springate, and Taffler models include CELHA, DERIM, DGKLB, DOKTA, IZDMC, MNDRS, PINSU, TUPRS, VKING.

As a result of these two (Altman and Springate) or three models (Altman, Springate and Taffler), a detailed study can be made about the financial ratios of companies with financial failure risk in all quarters. Therefore, a more robust model can be proposed for the risk of financial failure. While this will benefit companies in shaping their financial policies, it will enable them to reach more reliable results in their decision making. In the literature, financially unsuccessful and financially successful companies are examined, while financially unsuccessful companies are bankrupt. In this study, one notes that companies that have not gone bankrupt may also risk financial failure. For this reason, companies with and without the risk of financial failure can be identified with sector-specific financial ratios, and even different thresholds can be found.

\section{REFERENCES}

Aktaş, R. (1993). Endüstri İşletmeleri için Mali Başarısızlık Tahmini (Çok Boyutlu Model Uygulaması). 1. Baskı, Türkiye İş Bankası Kültür Yayınları, Ankara.

Aktaş, R., Doğanay, M. M. and Yıldız, B. (2003). "Mali Başarısızlığın Öngörülmesi: İstatistiksel Yöntemler ve Yapay Sinir Ağı Karşılaştırması”, Ankara Üniversitesi Sosyal Bilimler Fakültesi Dergisi, 58(4), 1-24.

Akyüz, K. C., Yıldırım, İ., Akyüz, İ. and Tugay, T. (2017). "Borsa İstanbul’da İşlem Gören Kâğıt ve Kâğıt Ürünleri Sanayi İşletmelerinin Finansal Başarısızlık Düzeylerinin Oran Analizi ve Diskriminant Analizi Yöntemleri Kullanılarak Ölçülmesi”, Journal of Forestry, 13(1), 60-74.

Almansour, B. Y. (2015). "Empirical Model for Predicting Financial Failure", American Journal of Economics, Finance and Management, 1(3), 113-124.

Altman, E. I. (1968). "Financial Ratios, Discriminant Analysis and the Prediction of Corporate Bankruptcy”, The Journal of Finance, 23(4), 589-609.

Altman, E. I. (1983). "Why Business Fail”, The Journal Of Business Strategy, 3(4), 15-22. 
Altman, E. I. (1993). Corporate Financial Distress: A Complete Guide to Predicting, Avoiding and Dealing with Bankruptcy. 2nd Edition, New York: Wiley and Sons.

Arslantürk Çöllü, D., Akgün, L. and Eyduran, E. (2020). "Karar Ağacı Algoritmalarıyla Finansal Başarısızlık Tahmini: Dokuma, Giyim Eşyası ve Deri Sektörü Uygulaması”, Uluslararası Ekonomi ve Yenilik Dergisi, 6(2), 225-246.

Beaver, W. H. (1966). "Financial Ratios as Predictors of Failure", Journal of Accounting Research, 4, 71-111.

Chen, J., Marshall, B. R., Zang, J. and Ganesh, S. (2006). "Financial Distress Prediction in China", Review of Pacific Basin Financial Markets and Policies, 9(2), 317-336.

Deloitte (2020). COVID-19 - Measures aiming to tackle the effects of the Coronavirus pandemic. April 2020, Tax Alert.

Ertan, A. S. and Ersan, Ö. (2018). "Finansal Başarısızlığı Belirleyen Etkenler: Türkiye Imalat Sektörü Örneği”, Marmara Üniversitesi İktisadi ve İdari Bilimler Dergisi, 40(2), 181-207.

Fejér-Király, G., Ágoston, N. and Varga, J. (2019). "Modelling the Financial Failure of Romanian Stock Companies", Acta Universitatis Sapientiae, Economics and Business, 7(1), 65-82.

Frydman, H., Altman, E. I. and Kao, D. L. (1985). "Introducing Recursive Partitioning for Financial Classification: The Case of Financial Distress", Journal of Finance, 40(1), 269-291.

Ganesalingam, S. and Kumar, K. (2001). "Detection of Financial Distress via Multivariate Statistical Analysis", Managerial Finance, 27(4), 45-55.

Göktan, E. (1981). Muhasebe Oranları Yardımıyla ve Diskriminant Analizi Tekniği Kullanılarak Endüstri İşletmelerinin Mali Başarısızlığının Tahmini Üzerine Ampirik Bir Araştırma. Doçentlik tezi, Ankara.

Gruszczynski, M. (2004). "Financial Distress of Companies in Poland", International Advances in Economic Research, 10(4), 249-256.

İçerli, M. Y. and Akkaya, G. C. (2006). "Finansal Açıdan Başarılı Olan İşletmelerle Başarısız Olan İşletmeler Arasında Finansal Oranlar Yardımıyla Farklılıkların Tespiti”, Atatürk Üniversitesi İktisadi ve İdari Bilimler Dergisi, 20(1), 413-421.

Ijaz, M. S., Hunjra, A. I., Hameed, Z., Maqbool, A. and Azam, R. (2013). "Assessing the Financial Failure Using Z-Score and Current Ratio: A Case of Sugar Sector Listed Companies of Karachi Stock Exchange", World Applied Sciences Journal, 23(6), 863-870.

Jones, S. and Hensher, D. A. (2004). "Predicting Firm Financial Distress: A Mixed Logit Model", The Accounting Review, 79(4), 1011-1038.

KAP (2021). "BIST Manufacturing Companies", available at: https://www.kap.org.tr/tr/Sektorler, (accessed 25 September 2021).

Karaca, S., and Özen, E. (2017). Financial failure estimation of companies in BIST tourism index by Altman Model and its effect on market prices. BRAND. Broad Research in Accounting, Negotiation, and Distribution, 8(2), 11-23.

PAGE 111| Journal of Corporate Governance, Insurance, and Risk Management | 2021, VOL. 8, Series. 2 
Karadeniz, E. and Öcek, C. (2019). "Finansal Başarısızlık Riski Taşıyan ve Taşımayan Işletmelerin Finansal Oranlarının Karşılaştırmalı Analizi: Borsa İstanbul Turizm Işletmelerinde Bir Araştırma”, Seyahat ve Otel İşletmeciliği Dergisi, 16(2), 191-206.

Keener, M. H. (2013). "Predicting The Financial Failure of Retail Companies in the United States", Journal of Business \& Economics Research, 11(8), 373-380.

Kelley, N. S. and Osterholm, M. T. (2008). "Pandemic influenza, electricity, and the coal supply chain: addressing crucial preparedness gaps in the United States. Center for Infectious Disease Research and Policy (CIDRAP)" University of Minnesota, available at: https://www.cidrap.umn.edu/sites/default/files/public/downloads/cidrap_coal_report.pdf (accessed 30 September 2021).

Li, L. and Faff, R. (2019). “Predicting Corporate Bankruptcy: What Matters?”, International Review of Economics and Finance, 62, 1-19.

Merwin, C. (1942). Financing Small Corporations: In Five Manifacturing Industries, 1926-36 New York: NationalBureau Of Economic Research.

Odom, M. D. and Sharda, R. (1990). "A Neural Network Model for Bankruptcy Prediction”, IJCNN international Joint Conference On Neural Networks, 17-21 June, Sand Diego, CA, USA, 163168.

Ohlson, J. A. (1980). "Financial Ratios and the Probabilistic Prediction of Bunkruptcy", Journal of Accounting Research, 18(1), 109-131.

Salehi, M. and Abedini, B. (2009). "Financial Distress Prediction in Emerging Market: Empirical Evidence from Iran”, Business Intelligence Journal, 2(2), 398-409.

Springate, G. L. (1978). Predicting the Possibility of Failure in a Canadian firm. British Columbia, Simon Fraser University, Canada.

Taffler, R. J. (1982). "Forecasting Company Failure in the UK Using Discriminant Analysis and Financial Ratio Data", Journal of the Royal Statistical Society, 145(3), 342-358.

Taffler, R. J. (1983). "The Assesment of Company Solvency and Performance Using A Statistical Model”, Accounting and Business Research, 13(52), 295-307.

Tamari, M. (1966). "Financial Raitos as Means of Forecasting Bankruptcy", Management International Review, 6(4), 15-21.

Temelli, F. and Tekin, B. (2020). "Finansal Başarısızlığın Springate Modeli ile Tahmin Edilmesi”, Edit. Güngör, B., Yerdelen Kaygın C., Finansal Performans Araştırmalarında Güncel Yaklaşımlar, Nobel Yayınevi, Ankara, 75-101.

Terzi, S. (2011). "Finansal Rasyolar Yardımıyla Finansal Başarısızlık Tahmini: Gıda Sektöründe Ampirik Bir Araştırma", Çukurova Üniversitesi İ̈BF Dergisi, 15(1), 1-18.

Tian, S. and Yu, Y. (2017). "Financial Bankruptcy Prediction: An International Evidence", International Review of Economics and Finance, 51, 510-526.

PAGE 112| Journal of Corporate Governance, Insurance, and Risk Management | 2021, VOL. 8, Series. 2 
Tirapat, S. and Nittayagasetwat, A. (1999). “An Investigation of Thai Listed Firms' Financial Distress Using Macro and Micro Variables", Multinational Finance Society, 3(2), 103-125.

Toraman, C. and Karaca, C. (2016). "Kimya Endüstrisinde Faaliyet Gösteren Firmalar Üzerinde Mali Başarısızlık Tahmini: Borsa İstanbul'da Bir Uygulama”, Muhasebe ve Finansman Dergisi, 70(Nisan), 111-128.

Ünsal, A. (2001). "Mali Başarılı ve Mali Başarısız Şirketlerin Ayrımını Sağlayan Diskriminant Fonksiyonunun Bulunması”, Çukurova Üniversitesi Sosyal Bilimler Enstitüsü Dergisi, 7(7), 214-234.

Ural, K., Gürarda, Ş. and Önemli, M. B. (2015). "Lojistik Regresyon Modeli ile Finansal Başarısızlık Tahminlemesi: Borsa İstanbul'da Faaliyet Gösteren Gıda, içki ve Tütün Şirketlerinde Uygulama”, Muhasebe ve Finansman Dergisi, 67(Temmuz), 85-100.

Weibel, P. F. (1973). The Value of Criteria to Judge Credit Worthiness in the Lending of Bank, Bern/Stuttgart Quoted in Choi F.D.S., International Finance and Accounting Handbook 3rd Edition, USA: Wiley \& Sons.

Winakor, A. and Smith, R.F. (1935). Changes in Financial Structure of Unsuccesful Industrial Companies, Bureau Of Business Research Bulletin, No: 51, Urbana: University Of Illinois Press.

Zeytinoğlu, E. and Akarım, Y. D. (2013). "Financial Failure Prediction Using Financial Ratios: An Empirical Application on İstanbul Stock Exchange”, Journal of Applied Finance \& Banking, 3(3), 107-116.

Zmijewski, M. E. (1984). "Methodological Issues Related to the Estimation of Financial Distress Prediction Models", Journal of Accounting Research, 22, 59-82. 